\title{
TIME-DISCRETIZATION OF STOCHASTIC 2-D NAVIER-STOKES EQUATIONS WITH A PENALTY-PROJECTION METHOD
}

\author{
TSIRY RANDRIANASOLO AND ERIKA HAUSENBLAS
}

\begin{abstract}
A time-discretization of the stochastic incompressible Navier-Stokes problem by penalty method is analyzed. Some error estimates are derived, combined, and eventually arrive at a speed of convergence in probability of order $1 / 4$ of the main algorithm for the pair of variables velocity and pressure. Also, using the law of total probability, we obtain the strong convergence of the scheme for both variables.
\end{abstract}

\section{INTRODUCTION}

Let $T>0$ and $\mathfrak{P}:=(\Omega, \mathcal{F}, \mathbb{F}, \mathbb{P})$ be a filtered probability space with the filtration $\mathbb{F}:=\left(\mathcal{F}_{t}\right)_{0 \leq t \leq T}$ satisfying the usual conditions. We refer to the following system of equations as the stochastic incompressible Navier-Stokes problem (SNS),

$$
\left\{\begin{aligned}
\boldsymbol{u}_{t}-v \Delta \boldsymbol{u}+[\boldsymbol{u} \cdot \nabla] \boldsymbol{u}+\nabla \mathrm{p} & =\dot{\boldsymbol{W}}, \text { in } \mathbb{R}^{2}, \\
\operatorname{div} \boldsymbol{u} & =0, \text { in } \mathbb{R}^{2} .
\end{aligned}\right.
$$

Here $\boldsymbol{u}=\{\boldsymbol{u}(t, \boldsymbol{x}): t \in[0, T]\}$ and $\mathrm{p}=\{\mathrm{p}(t, \boldsymbol{x}): t \in[0, T]\}$ are unknown stochastic processes on $\mathbb{R}^{2}$, representing respectively the velocity and the pressure of a fluid with kinematic viscosity $v$ filling the whole space $\mathbb{R}^{2}$, in each point of $\mathbb{R}^{2}$.

In $\mathbb{R}^{2}$, we endow (1.1) with an initial condition,

$$
u(0, x)=u_{0}(x)
$$

and periodic boundary conditions,

$$
\boldsymbol{u}\left(t, \boldsymbol{x}+L \boldsymbol{b}_{j}\right)=\boldsymbol{u}(t, x), \quad j=1,2, \quad t \in[0, T],
$$

where $u$ has a vanishing spatial average. Here $\left(\boldsymbol{b}_{1}, \boldsymbol{b}_{2}\right)$ is the canonical basis of $\mathbb{R}^{2}$ and $L>0$ is the period in the $j$ th direction; $D=(0, L) \times(0, L)$ is the square of the period. The term $\boldsymbol{W}:=\{\boldsymbol{W}(t): t \in$ $[0, T]\}$ is a $\mathcal{K}$-valued Wiener process where $\mathcal{K}$ is a separable Hilbert space.

An incompressible fluid flow is usually modeled with a deterministic Navier-Stokes equation. The stochastic Navier-Stokes (1.1) is a well known model that captures fluid instabilities under ambient noise [5] or small scales perturbation for homogeneous turbulent flow, see e.g. [3], [6], and [32].

Strong approximation of Stochastic Partial Differential Equations (SPDEs), such as Eq. (1.1), is mostly the natural approach because of its link with the numerical analysis of deterministic equations. However, this type of approximation is often inaccessible for nonlinear SPDEs. Indeed, when the nonlinearity is neither globally Lipschitz nor monotone, weak convergence or convergence in probability are frequently considered, see e.g. [2], [9], [16], [17], [22], [25], and [30]. Another notion, the speed of convergence in probability, was first put forward by Printems in [33] for some parabolic SPDEs. Regardless of the type of convergence, we may also have to consider different approaches according to the characteristic of the equation. In particular for the SNS, we can use e.g. a numerical approximation using an Ornstein-Uhlenbeck as an auxiliary step such as in [22], or using splitting methods such as in [4, 12], or using the Wiener chaos expansion such as in [25], or using the layer method (probabilistic representation) such as in [30]. Carelli and Prohl proved in [13] that a speed of

Date: May 3, 2018.

This research was supported by the Austrian Science Fund (FWF): P26958 and the German Research Council as part of the Collaborative Research Center SFB 1283. 
convergence in probability can be derived from some direct numerical approximations of the SNS. Here the convergence concerns only one variable, the velocity field.

The SNS shares the same complexity as its deterministic counter part, when it comes to computations. Velocity and pressure are both coupled by the incompressibility constraint, which often requires a saddle point problem to solve. To break this saddle point character of the system, velocity and pressure are decoupled by perturbing the divergence free condition by a penalty method [28, Chapter 3] and choosing a penalty operator in a similar fashion as in [15]. This consists, for every $\varepsilon>0$, to solve the penalized version of (1.1), i.e.

$$
\left\{\begin{aligned}
\boldsymbol{u}_{t}^{\varepsilon}-v \Delta \boldsymbol{u}^{\varepsilon}+\left[\boldsymbol{u}^{\varepsilon} \cdot \nabla\right] \boldsymbol{u}^{\varepsilon}+\frac{1}{2}\left(\operatorname{div} \boldsymbol{u}^{\varepsilon}\right) \boldsymbol{u}^{\varepsilon}+\nabla \mathrm{p}^{\varepsilon} & =\dot{\boldsymbol{W}}, \text { in } \mathbb{R}^{2}, \\
\operatorname{div} \boldsymbol{u}^{\varepsilon}+\varepsilon \mathrm{p}^{\varepsilon} & =0, \text { in } \mathbb{R}^{2} .
\end{aligned}\right.
$$

This belongs to a more general class of approximation methods for the Navier-Stokes equation, called projection and quasi-compressible methods. This includes the artificial compressibility method, the pressure stabilization, and the pressure correction method. For a complete survey or review on these methods, the reader is referred for instance to [24] or the monograph [34]. Even though these methods are already very popular and efficient in the deterministic framework, the paper of Carelli, Hausenblas, and Prohl, see [12], is the only work, which treats on projection and quasi-compressible methods for the stochastic Stokes equation by using the pressure stabilization and the pressure correction methods to derive an algorithm based on a time marching strategy. The artificial compressibility method has already been used to prove existence and pathwise uniqueness of global strong solutions of SNS, see [29], or adapted solutions to the backward SNS by a local monotonicity argument, see [41]. Concerning the penalty method, it has been introduced in [38] by Temam for the deterministic Navier-Stokes equations where he established its convergence. Since then, the method has been improved by Shen with the addition of error estimates in a sequence of papers including [36] and [37]. It has been used (with a different penalty operator) in a stochastic framework in [11] as an auxiliary step to prove the existence of a spatially homogeneous solution of a SNS driven by a spatially homogeneous Wiener random field.

In this paper, we study a semi-implicit time-discretization scheme for the full stochastic incompressible 2D Navier-Stokes equation based on the penalized system Eq. (1.2). Formally, the scheme consists of solving the following equations:

Given $0<\eta<1 / 2, \alpha>1, u_{0}, \phi^{0}=0$. For $\ell=1, \ldots, M$ :

- Step 1 (Penalization): Find $\tilde{\boldsymbol{u}}^{\ell}$ such that

$$
\tilde{\boldsymbol{u}}^{\ell}-v k \Delta \tilde{\boldsymbol{u}}^{\ell}+k \tilde{B}\left(\tilde{\boldsymbol{u}}^{\ell}, \tilde{\boldsymbol{u}}^{\ell}\right)-k^{1-\eta} \nabla \operatorname{div} \tilde{\boldsymbol{u}}^{\ell}=\Delta_{\ell} \boldsymbol{W}+\boldsymbol{u}^{\ell-1}-k \nabla \phi^{\ell-1} ;
$$

- Step 2: Find $\phi^{\ell}$ such that

$$
\Delta \phi^{\ell}=\Delta \phi^{\ell-1}+(\alpha k)^{-1} \operatorname{div} \tilde{\boldsymbol{u}}^{\ell} ;
$$

- Step 3 (Projection): $\boldsymbol{u}^{\ell}=\boldsymbol{P}_{\mathbb{H}} \tilde{\boldsymbol{u}}^{\ell}$, i.e.

$$
\boldsymbol{u}^{\ell}=\tilde{\boldsymbol{u}}^{\ell}-\alpha k \nabla\left(\phi^{\ell}-\phi^{\ell-1}\right), \quad \mathrm{p}^{\ell}=\widetilde{\mathrm{p}}^{\ell}+\phi^{\ell}+\alpha\left(\phi^{\ell}-\phi^{\ell-1}\right) .
$$

More details are given in Algorithm 1. We focus on the time-discretization, since different technical endeavors may obscure the main difficulty of the time-discretization. A paper which is similar to ours is [13], where the authors show the convergence in probability of a space-time discretization of stochastic incompressible Navier-Stokes in 2D. The numerical schemes they use are implicit/semiimplicit in time and use a divergence-free finite element pairing such as the Scott-Vogelius finite element for the velocity and the pressure. The proof needs also some a priori estimates of the approximate solution in $\mathbb{V}$, the divergence-free space with finite enstrophy. These estimates are obtained by 
means of the additional orthogonal property of the nonlinear term in 2D and under periodic boundary conditions, i.e. $\langle[\boldsymbol{u} . \nabla] \boldsymbol{u}, \Delta \boldsymbol{u}\rangle=0$ for each $\boldsymbol{u} \in \mathbb{V}$. As we see in Eq. (1.2), the approximate solution is only slightly compressible, thus $\boldsymbol{u}^{\varepsilon} \notin \mathbb{V}$. Even with the projection step added, the additional orthogonal property required in [13] is inapplicable here. To overcome this issue we use the classical decomposition of the SNS into an Ornstein-Uhlenbeck process and a deterministic SNS. This decomposition has already been used for different purpose, e.g. in [10], [18], [19], [21], and [22]. The algorithm depends on the spatial perturbation parameter $\varepsilon>0$, a stability preserving parameter $\alpha>1$, and the time-step $k$. If we fix $\varepsilon=k^{\eta}$ with some $0<\eta<1 / 2$ and with any $\alpha>1$, a speed of convergence in probability of order $1 / 4$ is obtained for both velocity and pressure. Then, by means of the law of total probability, we deduce strong convergence of the scheme for both variables velocity and pressure. In this context, we respond to the lack of results regarding (speed of) convergence for the pressure iterates from algorithms based on pseudo-compressible and projection method for stochastic (Navier)-Stokes equations addressed by [12].

This paper is organized as follows. In Section 2, we introduce the assumptions and notations used and review some of the basic facts of the SNS, which are important for the proof, such as the time regularity of the solution and present a splitting argument that will be used later on. In the Section 3, we develop stability of the main algorithm and derive error estimates for some auxiliary algorithms. In Section 4, we treat the speed of convergence in probability, then the strong convergence of the main algorithm.

\section{PRELIMINARIES}

In this section, we present the assumptions and notations used in this work. We also prove the time regularity of the pressure. As a preparatory work, before going into the numerical analysis, we formulate (1.1) according to the classical decomposition of the SNS into an Ornstein-Uhlenbeck process and a deterministic Navier-Stokes depending on a stochastic process.

2.1. Functional settings and notations. To introduce a spatial variable process, i.e. a vector-valued process to the Brownian motion $\boldsymbol{W}$, we introduce a family of mutually independent and identically distributed real-valued Brownian motions $\left\{\beta_{j}(t): t \in[0, T]\right\}, j \in \mathbb{N}$, and a covariance $Q$. If $Q \in \mathcal{L}(\mathcal{K})$ (the space of bounded linear operators from $\mathcal{K}$ to $\mathcal{K}$ ) is non-negative definite and symmetric with an orthonormal basis $\left\{\boldsymbol{d}_{j}: j \in \mathbb{N}\right\}$ of eigenfunctions with corresponding eigenvalues $q_{j} \geq 0$ such that $\sum_{j \in \mathbb{N}} q_{j}<\infty$, then $Q \in \mathcal{L}_{1}(\mathcal{K})$ (the space of trace-class operator on $\mathcal{K}$ ) and the series

$$
\mathbf{W}(t)=\sum_{j=1}^{\infty} \sqrt{q_{j}} \beta_{j}(t) \boldsymbol{d}_{j}, \quad \forall t \in[0, T]
$$

converges in $L^{2}(\Omega ; \mathcal{C}([0, T] ; \mathcal{K}))$ and it defines a $\mathcal{K}$-valued Wiener process with covariance operator $Q$ also called $Q$-Wiener process. Furthermore, for any $\ell \in \mathbb{N}$ there exists a constant $C_{\ell}>0$ such that

$$
\mathbb{E}\|\boldsymbol{W}(t)-\boldsymbol{W}(s)\|_{\mathcal{K}}^{2 \ell} \leq C_{\ell}(t-s)^{\ell}(\operatorname{Tr} \boldsymbol{Q})^{\ell}, \quad \forall t \in[0, T] \quad \text { and } \quad \forall s \in[0, t) .
$$

Let $\mathcal{H}$ be another separable Hilbert space. We define by $\mathcal{L}_{2}\left(\mathcal{K}_{Q}, \mathcal{H}\right)$ the space of Hilbert-Schmidt operator from $\mathcal{K}_{Q}$ to $\mathcal{H}$, where $\mathcal{K}_{Q}$ is the separable Hilbert space defined by $\mathcal{K}_{Q}:=Q^{1 / 2} \mathcal{K}$.

We can define the $\mathcal{H}$-valued Itô integral with respect to a $Q$-Wiener process $W$ by

$$
\int_{0}^{t} \Phi(s) d \mathbf{W}(s):=\sum_{j=1}^{\infty} \int_{0}^{t} \Phi(s) \sqrt{q_{j}} d_{j} d \beta_{j}(s), \quad \forall t \in[0, T]
$$

which is also a $\mathcal{H}$-valued martingale satisfying the Burkholder-Davis-Gundy inequality (see [26, Theorem 3.3.28]), given by

$$
\mathbb{E} \sup _{0 \leq s \leq t}\left\|\int_{0}^{s} \Phi(\tau) d \boldsymbol{W}(\tau)\right\|_{\mathcal{H}}^{2 r} \leq C_{r}\left(\int_{0}^{t}\|\Phi(\tau)\|_{\mathcal{L}_{2}\left(\mathcal{K}_{Q}, \mathcal{H}\right)}^{2} d \tau\right)^{r}, \forall t \in[0, T], \forall r>0 .
$$


In the case of scalar functions, we denote the usual Sobolev spaces by $W^{m, 2}(D)(m=0,1,2, \ldots, \infty)$. The corresponding scalar product and the corresponding norm for any nonnegative integer $m$ is denoted by

$$
(\boldsymbol{u}, \boldsymbol{v})_{m}=\int_{D} \sum_{\ell=0}^{m} \partial^{\ell} \boldsymbol{u} \partial^{\ell} \boldsymbol{v} d \boldsymbol{x} \quad \text { and } \quad\|\boldsymbol{u}\|_{m}=\|\boldsymbol{u}\|_{W^{m, 2}}=(\boldsymbol{u}, \boldsymbol{u})_{m}^{1 / 2} .
$$

By $W_{0}^{m, 2}(D)$, we denote the closure in $W^{m, 2}(D)$ of the space $\mathcal{C}_{0}^{\infty}(D)$ of all smooth functions defined on $D$ with compact support. Further, $W^{-m, 2}(D)$ is the space that is dual to $W^{m, 2}(D) \cap W_{0}^{1,2}(D)$. Particularly for $m=0$, the space $W^{m, 2}(D)$ is usually denoted by $L^{2}(D)$ and then the scalar product and norm are denoted simply by $(\cdot, \cdot)$ and $\|\cdot\|$, respectively. We reserve the notation $\langle\cdot, \cdot\rangle$ for the duality bracket. In general, we denote the usual Lebesgue spaces by $L^{p}, 1 \leq p \leq \infty$, which are endowed with the standard norms denoted by $\|\cdot\|_{L^{p}}$. We denote by $L_{\text {per }}^{p}$ and $W_{\text {per }}^{m, 2}$ the Lebesgue and Sobolev spaces of functions that are periodic and have vanishing spatial average, respectively. The spaces of vector-valued functions will be indicated with Blackboard bold letters, for instance $\mathbb{L}_{\text {per }}^{2}:=\left(L_{\text {per }}^{2}\right)^{2}$. In further analyses, we will not distinguish between the notation of inner products and norms in scalar or vector-valued applications.

The two spaces frequently used in the theory of Navier-Stokes equations are

$$
\mathbb{H}=\left\{\boldsymbol{v} \in \mathbb{L}_{\text {per }}^{2}(D): \operatorname{div} \boldsymbol{v}=0 \text { in } \mathbb{R}^{2}\right\} \quad \text { and } \quad \mathbb{V}=\left\{\boldsymbol{v} \in \mathbb{W}_{\text {per }}^{1,2}(D): \operatorname{div} \boldsymbol{v}=0 \text { in } \mathbb{R}^{2}\right\} .
$$

The space $\mathbb{V}$ is a Hilbert space with the scalar product $(\cdot, \cdot)_{1}$ and the Hilbert norm induced by $\mathbb{W}^{1,2}$.

Let $\boldsymbol{P}_{\mathbb{H}}$ denote the $\mathbb{L}^{2}$-projection on the space $\mathbb{H}$ also known as Helmholtz-Leray projector. As an orthogonal projection, it satisfies the following identity

$$
\left\langle\boldsymbol{P}_{\mathbb{H}} \boldsymbol{v}-\boldsymbol{v}, \boldsymbol{P}_{\mathbb{H}} \boldsymbol{v}\right\rangle=0, \quad \forall \boldsymbol{v} \in \mathbb{L}_{\text {per }}^{2} .
$$

The projection $\mathbb{P}_{\mathbb{H}}$ is continuous from $\mathbb{W}^{1,2}(D)_{0}$ into $\mathbb{W}^{1,2}(D)$ (cf. [40, Remark 1.6] and [7, Proposition IV.3.7.]) and we can find a positive constant $C=C(D)$ such that

$$
\left\|\boldsymbol{P}_{\mathbb{H}} \boldsymbol{u}\right\|_{1} \leq C\|\boldsymbol{u}\|_{1}, \quad \forall \boldsymbol{u} \in \mathbb{W}^{1,2}(D) .
$$

Due to the Helmholtz-Hodge-Leray decomposition, any function $\boldsymbol{u} \in \mathbb{L}^{2}(D)$ can be represented as $\boldsymbol{u}=\boldsymbol{P}_{\mathbb{H}} \boldsymbol{u}+\nabla \mathrm{q}$, where $\mathrm{q}$ is a scalar $D$-periodic function such that $\mathrm{q} \in L_{\mathrm{per}}^{2}(D)$. It is natural to introduce the notation $\boldsymbol{P}_{\mathbb{H}}^{\perp} \boldsymbol{u}:=\nabla \mathrm{q}$ and hence write

$$
\boldsymbol{u}=\boldsymbol{P}_{\mathbb{H}} \boldsymbol{u}+\boldsymbol{P}_{\mathbb{H}}^{\perp} \boldsymbol{u}, \quad \text { with } \quad \boldsymbol{P}_{\mathbb{H}}^{\perp} \boldsymbol{u} \in \mathbb{H}^{\perp}=\left\{\boldsymbol{v}: \boldsymbol{v} \in \mathbb{L}^{2}(D), \boldsymbol{v}=\nabla \mathrm{q}\right\} .
$$

With periodic boundary conditions the Stokes operator $\boldsymbol{A}=-\boldsymbol{P}_{\mathbb{H}} \Delta$ coincides with the Laplacian operator $-\Delta$. The operator $A$ can be seen as an unbounded positive linear selfadjoint operator on $\mathbb{H}$ with domain $\mathcal{D}(A)=\mathbb{W}^{2,2} \cap \mathbb{V}$. We can define the powers $A^{\alpha}, \alpha \in \mathbb{R}$, with domain $\mathcal{D}\left(A^{\alpha}\right)$. The norm $\left\|\boldsymbol{A}^{s / 2} \boldsymbol{u}\right\|$ on $\mathcal{D}\left(\boldsymbol{A}^{s / 2}\right)$ is equivalence to the norm induced by $\mathbb{W}_{0}^{s, 2}(D)$. In addition, we also have the following equivalence of norm:

Proposition 2.1 (Equivalence of norms). There exist positive numbers $c_{1}$ and $c_{2}$ such that $\forall \boldsymbol{u} \in \mathbb{H}$ :

(i) $\left\|\boldsymbol{A}^{-1} \boldsymbol{u}\right\|_{s} \leq c_{1}\|\boldsymbol{u}\|_{s-2}, \quad s=1,2$;

(ii) $c_{2}\|\boldsymbol{u}\|_{-1}^{2} \leq\left(\boldsymbol{A}^{-1} \boldsymbol{u}, \boldsymbol{u}\right) \leq c_{1}^{2}\|\boldsymbol{u}\|_{-1}^{2}$.

Proof. The reader is referred to [35, Equation (2.1)] or [34, Lemma 2.3] for the proof. It relies on the elliptic regularity of the Stokes operator and the definition of negative Sobolev norms.

We now introduce some operators usually associated with the Navier-Stokes equations and their approximations. In particular,

$$
\begin{aligned}
& \boldsymbol{B}(\boldsymbol{u}, \boldsymbol{v})=[\boldsymbol{u} \cdot \nabla] \boldsymbol{v}, \quad \tilde{\boldsymbol{B}}(\boldsymbol{u}, \boldsymbol{v})=\boldsymbol{B}(\boldsymbol{u}, \boldsymbol{v})+(\operatorname{div} \boldsymbol{u}) \boldsymbol{v} / 2, \\
& b(\boldsymbol{u}, \boldsymbol{v}, \boldsymbol{w})=\langle\boldsymbol{B}(\boldsymbol{u}, \boldsymbol{v}), \boldsymbol{w}\rangle, \quad \tilde{b}(\boldsymbol{u}, \boldsymbol{v}, \boldsymbol{w})=\langle\tilde{\boldsymbol{B}}(\boldsymbol{u}, \boldsymbol{v}), \boldsymbol{w}\rangle .
\end{aligned}
$$

The trilinear forms $b$ and $\tilde{b}$ satisfy the following properties: 
Skew-symmetry property

$$
\begin{array}{ll}
b(\boldsymbol{u}, \boldsymbol{v}, \boldsymbol{w})=-b(\boldsymbol{u}, \boldsymbol{w}, \boldsymbol{v}), & \boldsymbol{u} \in \mathbb{H} \text { and } \boldsymbol{v}, \boldsymbol{w} \in \mathbb{V}, \\
\tilde{b}(\boldsymbol{u}, \boldsymbol{v}, \boldsymbol{w})=-\tilde{b}(\boldsymbol{u}, \boldsymbol{w}, \boldsymbol{v}), & \boldsymbol{u}, \boldsymbol{v} \in \mathbb{W}^{1,2}(D) \text { and } \boldsymbol{w} \in \mathbb{W}_{\text {per }}^{1,2}(D) .
\end{array}
$$

Orthogonal property

$$
b(\boldsymbol{u}, \boldsymbol{v}, \boldsymbol{v})=0, \quad \forall \boldsymbol{u} \in \mathbb{H}, \forall \boldsymbol{v} \in \mathbb{W}_{\text {per }}^{1,2}(D) ; \quad \tilde{b}(\boldsymbol{u}, \boldsymbol{v}, \boldsymbol{v})=0, \quad \forall \boldsymbol{u}, \boldsymbol{v} \in \mathbb{W}_{\text {per }}^{1,2}(D) .
$$

The following estimates of the trilinear form $\tilde{b}$ will be used repeatedly in the upcoming sections. Let $v \in \mathbb{W}^{2,2}(D) \cap \mathbb{W}_{\text {per }}^{1,2}(D)$ and $\boldsymbol{u}, \boldsymbol{w} \in \mathbb{W}_{\text {per }}^{1,2}(D)$; a combination of integration by parts and Hölder inequality gives

$$
\tilde{b}(\boldsymbol{u}, \boldsymbol{v}, \boldsymbol{w}) \leq\|\boldsymbol{u}\|_{\mathbb{L}^{4}}\|\boldsymbol{v}\|_{1}\|\boldsymbol{w}\|_{\mathbb{L}^{4}} .
$$

From this estimate we can deduce using the Sobolev embedding $\mathbb{W}^{1,2}(D) \subset \mathbb{L}^{4}(D)$,

$$
\tilde{b}(\boldsymbol{u}, \boldsymbol{v}, \boldsymbol{w}) \leq C(L)\|\boldsymbol{u}\|_{1}\|\boldsymbol{v}\|_{1}\|\boldsymbol{w}\|_{1},
$$

or using the Ladyzhenskaya's inequality $\|\boldsymbol{u}\|_{\mathbb{L}^{4}} \leq C(L)\|\boldsymbol{u}\|^{1 / 2}\|\boldsymbol{u}\|_{1}^{1 / 2}$,

$$
\tilde{b}(\boldsymbol{u}, \boldsymbol{v}, \boldsymbol{w}) \leq C(L)\|\boldsymbol{u}\|^{1 / 2}\|\boldsymbol{u}\|_{1}^{1 / 2}\|\boldsymbol{v}\|_{1}\|\boldsymbol{w}\|^{1 / 2}\|\boldsymbol{w}\|_{1}^{1 / 2} .
$$

To find more about the above properties or additional properties of $b$ or $\tilde{b}$, and other estimates, the reader is referred to [39, Section 2.3].

2.2. General assumption and spatial regularity of the solution. In the following we choose $\mathcal{H}=$ $\mathbb{V}$, i.e. a solenoidal noise in SNS. An example of solenoidal noise is given in [12, Section 6]. We summarize the assumptions needed for data $\boldsymbol{W}, \boldsymbol{Q}$, and $\boldsymbol{u}_{0}$ :

$\left(S_{1}\right)$ For $\boldsymbol{Q} \in \mathcal{L}(\mathcal{K})$, let $\boldsymbol{W}=\{\boldsymbol{W}(t): t \in[0, T]\}$ be a $\boldsymbol{Q}$-Wiener process with values in a separable Hilbert space $\mathcal{K}$ defined on the stochastic basis $\mathfrak{P}$.

$\left(S_{2}\right) \boldsymbol{u}_{0} \in \mathbb{V}$.

In addition, we recall the notion of a strong solution to (1.1).

Definition 2.2 (Strong solution). Let $T>0$ be given and let Assumptions $\left(S_{1}\right)$ and $\left(S_{2}\right)$ be valid, with $\mathcal{H}=$ $\mathbb{V} . A \mathbb{V}$-valued process $\boldsymbol{u}=\{\boldsymbol{u}(t, \cdot): t \in[0, T]\}$ on $\left(\mathcal{F}_{t}\right)_{0 \leq t \leq T}$ is a strong solution to (1.1) if

(i) $\boldsymbol{u}(\cdot, \cdot, \omega) \in \mathcal{C}([0, T] ; \mathbb{V}) \cap L^{2}\left(0, T ; \mathbb{W}^{2,2} \cap \mathbb{V}\right) \mathbb{P}$-a.s.,

(ii) for every $t \in[0, T]$ and every $\varphi \in \mathbb{V}$, there holds $\mathbb{P}$-a.s.

$$
(\boldsymbol{u}(t), \boldsymbol{\varphi})+v \int_{0}^{t}(\nabla \boldsymbol{u}(s), \nabla \boldsymbol{\varphi})+b(\boldsymbol{u}(s), \boldsymbol{u}(s), \boldsymbol{\varphi}) d s=\left(\boldsymbol{u}_{0}, \boldsymbol{\varphi}\right)+\int_{0}^{t}(\boldsymbol{\varphi}, d \boldsymbol{W}(s)) .
$$

If Assumption $\left(S_{1}\right)$ holds and $\mathcal{H}=\mathbb{V}$, we can prove (cf. [20, Appendix 1]) that the solutions $\boldsymbol{u}$ of (1.1) as defined by Definition 2.2 satisfies for $2 \leq p<\infty$ the estimate

$$
\mathbb{E} \sup _{0 \leq t \leq T}\|\boldsymbol{u}(t)\|^{p}+v \mathbb{E}\left[\int_{0}^{T}\|\boldsymbol{u}(s)\|^{p-2}\|\nabla \boldsymbol{u}(s)\|^{2} d s\right] \leq C_{T, p}
$$

where $C_{T, p}=C_{T, p}\left(\operatorname{Tr} \boldsymbol{Q}, \mathbb{E}\left\|\boldsymbol{u}_{0}\right\|^{p}, \mathbb{E}\left\|\boldsymbol{u}_{0}\right\|_{\mathbb{V}}^{p}\right)>0$. In addition to the above estimate, if Assumption $\left(S_{2}\right)$ holds for $2 \leq p<\infty$, it is proven in [13, Lemma 2.1] that $u$ satisfies also the estimates

$$
\begin{array}{r}
\sup _{0 \leq t \leq T} \mathbb{E}\|\boldsymbol{u}(t)\|_{\mathbb{V}}^{p}+v \mathbb{E}\left[\int_{0}^{T}\|\boldsymbol{u}(s)\|_{\mathbb{V}}^{p-2}\|\boldsymbol{A} \boldsymbol{u}(s)\|^{2} d s\right] \leq C_{T, p} \\
\text { and } \mathbb{E} \sup _{0 \leq t \leq T}\|\boldsymbol{u}(t)\|_{\mathbb{V}}^{p} \leq C_{T, p} .
\end{array}
$$

We associate a pressure $\mathrm{p}$ to the velocity $\boldsymbol{u}$ by using a generalization of the de Rham theorem to processes, see [27, Theorem 4.1]. In addition, we also have the following estimate for the pressure: 
Proposition 2.3. Under Assumptions $\left(S_{1}\right)$ and $\left(S_{2}\right)$, there exists a constant $C>0$ such that the velocity fields $\boldsymbol{u}$ and pressure fields $\mathrm{p}$ satisfy $\mathbb{P}$-a.s.

$$
\|\mathrm{p}(t)\| \leq C\left\|A^{1 / 2} \boldsymbol{u}(t)\right\|^{2}, \quad \forall t \in[0, T] .
$$

Proof. To show the Proposition 2.3 we project equation (1.1) into $\mathbb{H}^{\perp}$ using the projection operator $\boldsymbol{P}_{\mathbb{H}}^{\perp}$. Since $\boldsymbol{P}_{\mathbb{H}}^{\perp}$ commutes with the Laplacian operator (we work with a periodic boundary condition) and $\operatorname{div} \boldsymbol{u}=0$, then

$$
\boldsymbol{P}_{\mathbb{H}}^{\perp} \boldsymbol{u}_{t}=0 \quad \text { and } \quad \boldsymbol{P}_{\mathbb{H}}^{\perp} \Delta \boldsymbol{u}=0 .
$$

In Assumption $\left(S_{1}\right)$ we suppose that the forcing term is divergence-free, hence, each solenoidale term vanishes after projection with $\boldsymbol{P}_{\mathbb{H}}^{\perp}$. The remaining terms give

$$
\nabla \mathrm{p}(t)=-\boldsymbol{P}_{\mathbb{H}}^{\perp} \boldsymbol{B}(\boldsymbol{u}(t), \boldsymbol{u}(t)), \quad \forall t \in[0, T] .
$$

It follows from [23, Lemma 2.2] for $r=2, n=2, \delta=1 / 2, \theta=\rho=1 / 2$ and (2.11) that

$$
\begin{aligned}
\|\nabla \mathrm{p}(t)\|_{-1}=\left\|\boldsymbol{P}_{\mathbb{H}}^{\perp} \boldsymbol{B}(\boldsymbol{u}(t), \boldsymbol{u}(t))\right\|_{-1} & \leq\left\|\boldsymbol{P}_{\mathbb{H}} \boldsymbol{B}(\boldsymbol{u}(t), \boldsymbol{u}(t))\right\|_{-1}+\|\boldsymbol{B}(\boldsymbol{u}(t), \boldsymbol{u}(t))\|_{-1} \\
& \leq C\left\|\boldsymbol{A}^{1 / 2} \boldsymbol{u}(t)\right\|^{2} .
\end{aligned}
$$

Finally, it follows by the Nečas inequality for functions with vanishing spatial average (cf. [7, Proposition IV.1.2.]), that there exists a constant $C>0$, such that

$$
\|\mathrm{p}(t)\| \leq C\left\|A^{1 / 2} \boldsymbol{u}(t)\right\|^{2}, \quad \forall t \in[0, T] .
$$

The constant $C$ comes from the Nečas inequality, more precisely from the definition of the norm in $\mathbb{W}^{-1,2}$ by the Fourier transform. Therefore, $C$ depends on the spatial dimension $d$ and the $L^{p_{-}}$ estimates for the Fourier transform multipliers. Here we have $d=2$ and $p=2$, but a similar estimate can be obtained for $d \geq 2$ and $2 \leq p<\infty$, see [14, Corollaries 1 and 2] and [31, Lemma 7.1].

\subsection{Regularity in time of the solution of the SNS.}

Lemma 2.4. Suppose that Assumption $\left(S_{1}\right)$ holds, and $\mathcal{H}=\mathbb{V}$. For the solution of (1.1), with $\boldsymbol{u}_{0} \in \mathbb{V}, 2 \leq$ $p<\infty$, we can find a constant $C=C(T, p, L)>0$, such that for $0 \leq s<t \leq T$ we have
(i) $\mathbb{E}\|\boldsymbol{u}(s)-\boldsymbol{u}(t)\|_{\mathbb{L}^{4}}^{p} \leq C|s-t|^{\eta p} \quad \forall 0<\eta<\frac{1}{2}$,
(ii) $\mathbb{E}\|\boldsymbol{u}(s)-\boldsymbol{u}(t)\|_{\mathbb{V}}^{p} \leq C|s-t|^{\frac{\eta p}{2}} \quad \forall 0<\eta<\frac{1}{2}$,
(iii) $\mathbb{E}\|\mathrm{p}(s)-\mathrm{p}(t)\|^{\frac{p}{2}} \leq C|s-t|^{\frac{\eta p}{4}} \quad \forall 0<\eta<\frac{1}{2}$.

Proof. The assertions $(i)$ and (ii) are direct quotations of [13, Lemma 2.3]. We only prove the assertion (iii). Let $t \in[0, T]$. Applying the projection $\boldsymbol{P}_{\mathbb{H}}^{\perp}$ on (1.1) we get

$$
\nabla \mathrm{p}(t)=-\mathbb{P}_{\mathbb{H}}^{\perp} \boldsymbol{B}(\boldsymbol{u}(t), \boldsymbol{u}(t)) .
$$

The following identity holds for $0 \leq s<t$

$$
\nabla(\mathrm{p}(s)-\mathrm{p}(t))=\mathbb{P}_{\mathbb{H}}^{\perp} \boldsymbol{B}(\boldsymbol{u}(t), \boldsymbol{u}(t)-\boldsymbol{u}(s))+\mathbb{P}_{\mathbb{H}}^{\perp} \boldsymbol{B}(\boldsymbol{u}(t)-\boldsymbol{u}(s), \boldsymbol{u}(s)) .
$$

Using the Nečas inequality for vanishing spatial average and Proposition 2.3, we obtain

$$
\begin{aligned}
\|\mathrm{p}(s)-\mathrm{p}(t)\| & \leq\left\|\mathbb{P}_{\mathbb{H}}^{\perp} \boldsymbol{B}(\boldsymbol{u}(t), \boldsymbol{u}(t)-\boldsymbol{u}(s))\right\|_{-1}+\left\|\mathbb{P}_{\mathbb{H}}^{\perp} \boldsymbol{B}(\boldsymbol{u}(t)-\boldsymbol{u}(s), \boldsymbol{u}(s))\right\|_{-1} \\
& \leq C\|\boldsymbol{u}(t)\|_{1}\|\boldsymbol{u}(t)-\boldsymbol{u}(s)\|_{1}+C\|\boldsymbol{u}(t)-\boldsymbol{u}(s)\|_{1}\|\boldsymbol{u}(s)\|_{1} .
\end{aligned}
$$

Taking the $p$ /2-moment and using the Hölder inequality we get

$$
\mathbb{E}\|\mathrm{p}(s)-\mathrm{p}(t)\|^{p / 2} \leq C(L)\left[\left(\mathbb{E}\|\boldsymbol{u}(t)\|_{1}^{p}\right)^{1 / 2}+\left(\mathbb{E}\|\boldsymbol{u}(s)\|_{1}^{p}\right)^{1 / 2}\right]\left(\mathbb{E}\|\boldsymbol{u}(t)-\boldsymbol{u}(s)\|_{1}^{p}\right)^{1 / 2} .
$$

We deduce from (2.13) and the assertion $(i)$ of the present lemma that

$$
\mathbb{E}\|\mathrm{p}(s)-\mathrm{p}(t)\|^{p / 2} \leq C_{T, 2}(L)|s-t|^{\eta p / 4} .
$$


2.4. Classical decomposition of the solution. Before going to the next section we introduce a splitting argument which is essential for the rest of the paper. We consider the auxiliary Stokes equation

$$
\left\{\begin{aligned}
d z+[-v \Delta z+\nabla \pi] d t & =d \boldsymbol{W}, \text { in } \mathbb{R}^{2}, \\
\operatorname{div} z & =0, \text { in } \mathbb{R}^{2},
\end{aligned}\right.
$$

with $z(0)=0$ and which corresponds the penalized system

$$
\left\{\begin{aligned}
d z^{\varepsilon}+\left[-v \Delta z^{\varepsilon}+\nabla \pi^{\varepsilon}\right] d t & =d W, \text { in } \mathbb{R}^{2}, \\
\operatorname{div} z^{\varepsilon}+\varepsilon \pi^{\varepsilon} & =0, \text { in } \mathbb{R}^{2},
\end{aligned}\right.
$$

with $z^{\varepsilon}(0)=0$.

As already pointed out by [13], the nonlinear term of the SNS does not allow to use a Gronwall argument. To tackle this issue, we use the classical decomposition of the solution $u$ into two parts: one part, given by the process $z$, will be random, but linear; the other part, denoted by $v$, will be nonlinear, but deterministic. In this way, we write the solution of (1.1) as $\boldsymbol{u}=\boldsymbol{v}+\boldsymbol{z}$, where $\boldsymbol{v}$ solves

$$
\left\{\begin{aligned}
\frac{d v}{d t}+\tilde{B}(v+z, v+z)-v \Delta v+\nabla \rho & =0, \text { in } \mathbb{R}^{2}, \\
\operatorname{div} \boldsymbol{v} & =0, \text { in } \mathbb{R}^{2},
\end{aligned}\right.
$$

with $\boldsymbol{v}(0)=\boldsymbol{u}_{0}$. The corresponding penalized system

$$
\left\{\begin{array}{r}
\frac{d \boldsymbol{v}^{\varepsilon}}{d t}+\tilde{\boldsymbol{B}}\left(\boldsymbol{v}^{\varepsilon}+\boldsymbol{z}^{\varepsilon}, \boldsymbol{v}^{\varepsilon}+\boldsymbol{z}^{\varepsilon}\right)-v \Delta \boldsymbol{v}^{\varepsilon}+\nabla \rho^{\varepsilon}=0, \text { in } \mathbb{R}^{2}, \\
\operatorname{div} \boldsymbol{v}^{\varepsilon}+\varepsilon \rho^{\varepsilon}=0, \text { in } \mathbb{R}^{2},
\end{array}\right.
$$

with $\boldsymbol{v}^{\varepsilon}(0)=\boldsymbol{u}_{0}$.

The system (2.21) (resp. (2.22)) are interpreted as deterministic equations which solves $v$ (resp. $\boldsymbol{v}^{\varepsilon}$ ) for a given random process $z$ (resp. $z^{\varepsilon}$ ).

\section{MAIN ALGORITHM AND AUXILIARY RESULTS}

We consider a time discretization of (1.1) based on the penalized system Eq. (1.2). For that purpose we fix $M \in \mathbb{N}$ and introduce an equidistant partition $I_{k}:=\left\{t_{\ell}: 1 \leq \ell \leq M\right\}$ covering $[0, T]$ with meshsize $k=T / M>0, t_{0}=0$, and $t_{M}=T$. Here the increment $\Delta_{\ell} \boldsymbol{W}:=\boldsymbol{W}\left(t_{\ell}\right)-\boldsymbol{W}\left(t_{\ell-1}\right) \sim \mathcal{N}(0, k Q)$ and we choose an uniform mesh size $k:=t_{\ell+1}-t_{\ell}$. For every $t \in\left[t_{\ell-1}, t_{\ell}\right]$ and all $\varphi \in \mathbb{W}_{\text {per, }}^{1,2}$, there hold $\mathbb{P}$-a.s.,

$$
\begin{aligned}
& \left(\boldsymbol{u}\left(t_{\ell}\right)-\boldsymbol{u}\left(t_{\ell-1}\right), \boldsymbol{\varphi}\right)+v \int_{t_{\ell-1}}^{t_{\ell}}(\nabla \boldsymbol{u}(s), \nabla \boldsymbol{\varphi}) d s \\
& +\int_{t_{\ell-1}}^{t_{\ell}} \tilde{b}(\boldsymbol{u}(s), \boldsymbol{u}(s), \boldsymbol{\varphi}) d s+\int_{t_{\ell-1}}^{t_{\ell}}(\nabla \mathrm{p}(s), \boldsymbol{\varphi}) d s=\int_{t_{\ell-1}}^{t_{\ell}}(\boldsymbol{\varphi}, d \boldsymbol{W}(s)), \\
& \left(\operatorname{div} \boldsymbol{u}\left(t_{\ell}\right), \chi\right)=0 .
\end{aligned}
$$

Note that instead of $b$ we use $\tilde{b}$. We can switch between both without any confusion since for each $s \in[0, T], \boldsymbol{u}(s) \in \mathbb{H}$.

Now we discretize the penalized system Eq. (1.2) instead of the original equation and project the result into $\mathbb{H}$. We derive the following algorithm:

Algorithm 1 (Main algorithm). Assume $\boldsymbol{u}^{\varepsilon, 0}:=\boldsymbol{u}_{0}$ with $\left\|\boldsymbol{u}_{0}\right\| \leq C$. Find for every $\ell \in\{1, \ldots, M\}$ a pair of random variables $\left(\boldsymbol{u}^{\varepsilon, \ell}, \mathbf{p}^{\varepsilon, \ell}\right)$ with values in $\mathbb{W}_{\text {per }}^{1,2} \times L_{\text {per }}^{2}$, such that we have $\mathbb{P}$-a.s.

- Penalization:

$$
\begin{gathered}
\left(\tilde{\boldsymbol{u}}^{\varepsilon, \ell}-\boldsymbol{u}^{\varepsilon, \ell-1}, \boldsymbol{\varphi}\right)+v k\left(\nabla \tilde{\boldsymbol{u}}^{\varepsilon, \ell}, \nabla \boldsymbol{\varphi}\right)+k \tilde{b}\left(\tilde{\boldsymbol{u}}^{\varepsilon, \ell}, \tilde{\boldsymbol{u}}^{\varepsilon, \ell}, \boldsymbol{\varphi}\right) \\
+k\left(\nabla \tilde{\boldsymbol{p}}^{\varepsilon, \ell}, \boldsymbol{\varphi}\right)+k\left(\nabla \phi^{\varepsilon, \ell-1}, \boldsymbol{\varphi}\right)=\left(\Delta_{\ell} \boldsymbol{W}, \boldsymbol{\varphi}\right), \forall \boldsymbol{\varphi} \in \mathbb{W}_{\mathrm{per}}^{1,2}, \\
\left(\operatorname{div} \tilde{\boldsymbol{u}}^{\varepsilon, \ell}, \chi\right)+\varepsilon\left(\tilde{\boldsymbol{p}}^{\varepsilon, \ell}, \chi\right)=0, \forall \chi \in L_{\text {per }}^{2} ;
\end{gathered}
$$


- Projection:

$$
\begin{aligned}
\left(\boldsymbol{u}^{\varepsilon, \ell}-\tilde{\boldsymbol{u}}^{\varepsilon, \ell}, \boldsymbol{\varphi}\right)+\alpha k\left(\nabla\left(\phi^{\varepsilon, \ell}-\phi^{\varepsilon, \ell-1}\right), \boldsymbol{\varphi}\right) & =0, \forall \boldsymbol{\varphi} \in \mathbb{W}_{\text {per }}^{1,2} \\
\left(\operatorname{div} \boldsymbol{u}^{\varepsilon, \ell}, \chi\right) & =0, \forall \chi \in L_{\text {per }}^{2} \\
\mathrm{p}^{\varepsilon, \ell} & =\tilde{\boldsymbol{p}}^{\varepsilon, \ell}+\phi^{\varepsilon, \ell}+\alpha\left(\phi^{\varepsilon, \ell}-\phi^{\varepsilon, \ell-1}\right) .
\end{aligned}
$$

Proposition 3.1. There exist iterates $\left\{\boldsymbol{u}^{\ell}: 1 \leq \ell \leq M\right\}$ which solve (3.25) and (3.26) at each time-step. Moreover, for every integer $l$, with $1 \leq \ell \leq M, \boldsymbol{u}^{\ell}$ is a $\mathcal{F}_{t_{\ell}}$-measurable.

Proof. Let us fix $\omega \in \Omega$. We use Lax-Milgram fixed-point theorem to show the existence of a $\mathbb{V}$-valued sequence $\left\{\boldsymbol{u}^{\varepsilon, \ell}: 1 \leq \ell \leq M\right\}$.

- Penalization: Since $\boldsymbol{u}^{\varepsilon, 0}$ and $\phi^{0}$ are given, and $\left|\Delta_{\ell} \boldsymbol{W}(\omega)\right|_{\mathcal{K}}<\infty$ for all $\ell \in\{1, \ldots, M\}$, we assume that $\widetilde{\boldsymbol{u}}^{\varepsilon, 1}(\omega), \ldots, \widetilde{\boldsymbol{u}}^{\varepsilon, \ell-1}(\omega)$ are also given. To find the pair of random variables $\left(\boldsymbol{u}^{\varepsilon, \ell}, \mathrm{p}^{\varepsilon, \ell}\right)$ in Algorithm 1 we need first to solve a nonlinear, nonsymmetric variational problem. Therefore, let us denote by $\mathcal{A}$ the nonlinear operator from $\mathbb{V}$ to $\mathbb{V}^{\prime}\left(\mathbb{V}^{\prime}\right.$ : dual of $\left.\mathbb{V}\right)$ defined by:

$$
\begin{aligned}
\left\langle\mathcal{A} \tilde{\boldsymbol{u}}^{\varepsilon, \ell}(\omega), \boldsymbol{w}(\omega)\right\rangle=\tilde{\boldsymbol{u}}^{\varepsilon, \ell}(\omega) & +v\left(\nabla \tilde{\boldsymbol{u}}^{\varepsilon, \ell}(\omega), \nabla \boldsymbol{w}^{\ell}(\omega)\right) \\
& +\tilde{b}\left(\tilde{\boldsymbol{u}}^{\varepsilon, \ell}(\omega), \tilde{\boldsymbol{u}}^{\varepsilon, \ell}(\omega), \boldsymbol{w}^{\ell}(\omega)\right), \quad \forall \boldsymbol{w}^{\ell}((\omega)) \in \mathbb{V} .
\end{aligned}
$$

Because $\tilde{b}$ satisfies the orthogonal property (2.8), then putting $\boldsymbol{w}^{\ell}=\tilde{u}^{\varepsilon, \ell}$ in (3.29) we thus have

$$
\left\langle\mathcal{A} \tilde{\boldsymbol{u}}^{\varepsilon, \ell}(\omega), \boldsymbol{w}(\omega)\right\rangle \geq v\left\|\boldsymbol{u}^{\varepsilon, \ell}(\omega)\right\|_{\mathbb{V}}^{2} .
$$

The operator $\mathcal{A}$ is therefore $\mathbb{V}$-elliptic and the Lax-Milgram theorem allows us to infer the existence of a unique solution of (3.29).

- Projection: If we take $\varphi=\nabla \phi^{\varepsilon, \ell}$ in (3.27) we see that this step is actually a Poisson problem. Since $\boldsymbol{u}^{\varepsilon, \ell}$ is given from the previous step, the existence of a unique solution $\phi^{\varepsilon, \ell}$ is deduced from the ellipticity of the Laplacian operator.

Since $\boldsymbol{u}^{\varepsilon, \ell}=\tilde{\boldsymbol{u}}^{\varepsilon, \ell}-\alpha k \nabla\left(\phi^{\varepsilon, \ell}-\phi^{\varepsilon, \ell-1}\right)$ and $\mathrm{p}^{\varepsilon, \ell}=\tilde{\mathrm{p}}^{\varepsilon, \ell}+\phi^{\varepsilon, \ell}+\alpha\left(\phi^{\varepsilon, \ell}-\phi^{\varepsilon, \ell-1}\right)$, the existence of a unique $\boldsymbol{u}^{\varepsilon, \ell}$ and $\mathrm{p}^{\varepsilon, \ell}$ follows.

The proof of the $\mathcal{F}_{t_{\ell}}$-measurability of $\tilde{\boldsymbol{u}}^{\varepsilon, \ell}$ and $\phi^{\varepsilon, \ell}$ can be done in a similar fashion as in [16], see also [2]. Since $\boldsymbol{u}^{\varepsilon, \ell}$ (resp. $\mathrm{p}^{\varepsilon, \ell}$ ) are obtained from $\tilde{\boldsymbol{u}}^{\varepsilon, \ell}$ (resp. $\phi^{\varepsilon, \ell}$ ) we also obtain their $\mathcal{F}_{t_{\ell}}$-measurability.

3.1. Stability. This section is inspired by [9, Lemma 3.1] and [13, Lemma 3.1]. Here we consider a coupled system, the first one is derived from the penalization and the second one is a projection step.

Lemma 3.2. Let $\phi^{\varepsilon, 0}=0$. Suppose that Assumptions $\left(S_{1}\right)$ and $\left(S_{2}\right)$ are valid with $\left\|\boldsymbol{u}^{0}\right\| \leq C$. Then, there exists a positive constant $C=C\left(L, T, \boldsymbol{u}^{0}, v\right)$ so that for every $\varepsilon>0$ and $\alpha>1$, the iterates $\left\{\boldsymbol{u}^{\varepsilon, \ell}: 1 \leq \ell \leq M\right\}$ solving Algorithm 1 and the intermediary iterates $\left\{\tilde{\boldsymbol{u}}^{\varepsilon, \ell}: 1 \leq \ell \leq M\right\},\left\{\tilde{\boldsymbol{p}}^{\varepsilon, \ell}: 1 \leq \ell \leq M\right\}$, and $\left\{\phi^{\varepsilon, \ell}: 1 \leq \ell \leq M\right\}$ satisfy for $q=1$ or $q=2$ :

$$
\begin{aligned}
& \text { (i) } v \mathbb{E}\left(k \sum_{\ell=1}^{M}\left\|\nabla \tilde{\boldsymbol{u}}^{\varepsilon, \ell}\right\|^{2}\left\|\boldsymbol{u}^{\varepsilon, \ell}\right\|^{2^{q}-2}\right) \leq C, \\
& \text { (ii) } k^{2} \mathbb{E} \max _{1 \leq m \leq M}\left\|\nabla \phi^{\varepsilon, m}\right\|^{2}\left\|\boldsymbol{u}^{\varepsilon, m}\right\|^{2^{q}-2}+\varepsilon \mathbb{E}\left(k \sum_{\ell=1}^{M}\left\|\tilde{\boldsymbol{p}}^{\varepsilon, \ell}\right\|\left\|\boldsymbol{u}^{\varepsilon, \ell}\right\|^{2^{q}-2}\right) \leq C, \\
& \text { (iii) } \mathbb{E} \max _{1 \leq m \leq M}\left\|\boldsymbol{u}^{\varepsilon, m}\right\|^{2^{q}}+v \mathbb{E}\left(k \sum_{\ell=1}^{M}\left\|\nabla \boldsymbol{u}^{\varepsilon, \ell}\right\|^{2}\left\|\boldsymbol{u}^{\varepsilon, \ell}\right\|^{2^{q}-2}\right) \leq C .
\end{aligned}
$$

Proof. The proof consits of three steps. First, we give some preparatory estimates. Then, we handle the case $q=1$, and, fially, we handle the case $q=2$. 
Step (I) Preparatory estimate. We take $\varphi=2 \tilde{\boldsymbol{u}}^{\varepsilon, \ell}$ in Eq. (3.25) and $\chi=\operatorname{div} \tilde{\boldsymbol{u}}^{\varepsilon, \ell}$ in Eq. (3.26), and use the orthogonal property (2.8) of $\tilde{b}$, to get

$$
\begin{aligned}
\left(\tilde{\boldsymbol{u}}^{\varepsilon, \ell}-\boldsymbol{u}^{\varepsilon, \ell-1}, \tilde{\boldsymbol{u}}^{\varepsilon, \ell}\right)+2 v k\left\|\nabla \tilde{\boldsymbol{u}}^{\varepsilon, \ell}\right\|^{2}+2 k\left(\nabla \tilde{\boldsymbol{p}}^{\varepsilon, \ell}, \boldsymbol{u}^{\varepsilon, \ell}\right) & \\
+2 k\left(\nabla \phi^{\varepsilon, \ell-1}, \tilde{\boldsymbol{u}}^{\varepsilon, \ell}\right) & =2\left(\Delta_{\ell} \boldsymbol{W}, \tilde{\boldsymbol{u}}^{\varepsilon, \ell}\right), \\
\left(\nabla \tilde{\boldsymbol{p}}^{\varepsilon, \ell}, \tilde{\boldsymbol{u}}^{\varepsilon, \ell}\right) & =\frac{1}{\varepsilon}\left\|\operatorname{div} \tilde{\boldsymbol{u}}^{\varepsilon, \ell}\right\|^{2} .
\end{aligned}
$$

Using the algebraic identity

$$
2(a-b) a=a^{2}-b^{2}+(a-b)^{2}
$$

in (3.30) we obtain

$$
\begin{aligned}
\left\|\tilde{\boldsymbol{u}}^{\varepsilon, \ell}\right\|^{2}-\left\|\boldsymbol{u}^{\varepsilon, \ell-1}\right\|^{2}+\left\|\tilde{\boldsymbol{u}}^{\varepsilon, \ell}-\tilde{\boldsymbol{u}}^{\varepsilon, \ell-1}\right\|^{2} & +2 v k\left\|\nabla \tilde{\boldsymbol{u}}^{\varepsilon, \ell}\right\|^{2}+2 \varepsilon k\left\|\tilde{\boldsymbol{p}}^{\varepsilon, \ell}\right\|^{2} \\
& +2 k\left(\nabla \phi^{\varepsilon, \ell-1}, \tilde{\boldsymbol{u}}^{\varepsilon, \ell}\right)=2\left(\Delta_{\ell} \boldsymbol{W}, \tilde{\boldsymbol{u}}^{\varepsilon, \ell}\right) .
\end{aligned}
$$

Let $\alpha>0$. We take $\boldsymbol{\varphi}=2 \tilde{\boldsymbol{u}}^{\varepsilon, \ell}$ in (3.27) and obtain

$$
\frac{\alpha-1}{\alpha}\left(\left\|\boldsymbol{u}^{\varepsilon, \ell}\right\|^{2}-\left\|\tilde{\boldsymbol{u}}^{\varepsilon, \ell}\right\|^{2}+\left\|\boldsymbol{u}^{\varepsilon, \ell}-\tilde{\boldsymbol{u}}^{\varepsilon, \ell}\right\|^{2}\right)=0 .
$$

Then, we take $\boldsymbol{\varphi}=\boldsymbol{u}^{\varepsilon, \ell}+\tilde{\boldsymbol{u}}^{\varepsilon, \ell}$ in (3.27) and obtain

$$
\frac{1}{\alpha}\left(\left\|\boldsymbol{u}^{\varepsilon, \ell}\right\|^{2}-\left\|\tilde{\boldsymbol{u}}^{\varepsilon, \ell}\right\|^{2}\right)+\frac{k}{2}\left(\nabla\left(\phi^{\varepsilon, \ell}-\phi^{\varepsilon, \ell-1}\right), \tilde{\boldsymbol{u}}^{\varepsilon, \ell}\right)=0 .
$$

Collecting together (3.32) to (3.34) we obtain

$$
\begin{aligned}
\left\|\boldsymbol{u}^{\varepsilon, \ell}\right\|^{2}-\left\|\boldsymbol{u}^{\varepsilon, \ell-1}\right\|^{2} & +\left\|\tilde{\boldsymbol{u}}^{\varepsilon, \ell}-\tilde{\boldsymbol{u}}^{\varepsilon, \ell-1}\right\|^{2}+\frac{\alpha-1}{\alpha}\left\|\boldsymbol{u}^{\varepsilon, \ell}-\tilde{\boldsymbol{u}}^{\varepsilon, \ell}\right\|^{2}+2 v k\left\|\nabla \tilde{\boldsymbol{u}}^{\varepsilon, \ell}\right\|^{2} \\
& +2 \varepsilon k\left\|\tilde{\boldsymbol{p}}^{\varepsilon, \ell}\right\|^{2}+k\left(\nabla\left(\phi^{\varepsilon, \ell-1}+\phi^{\varepsilon, \ell}\right), \tilde{\boldsymbol{u}}^{\varepsilon, \ell}\right) \leq 2\left(\Delta_{\ell} \boldsymbol{W}, \tilde{\boldsymbol{u}}^{\varepsilon, \ell}\right) .
\end{aligned}
$$

We take $\boldsymbol{\varphi}=\nabla\left(\phi^{\varepsilon, \ell}+\phi^{\varepsilon, \ell-1}\right)$ in (3.27) and obtain

$$
\left(\nabla\left(\phi^{\varepsilon, \ell}+\phi^{\varepsilon, \ell-1}\right), \tilde{u}^{\varepsilon, \ell}\right)=\alpha k\left\|\nabla \phi^{\varepsilon, \ell}\right\|^{2}-\alpha k\left\|\nabla \phi^{\varepsilon, \ell-1}\right\|^{2} .
$$

This implies,

$$
\begin{gathered}
\left\|\boldsymbol{u}^{\varepsilon, \ell}\right\|^{2}-\left\|\boldsymbol{u}^{\varepsilon, \ell-1}\right\|^{2}+\left\|\tilde{\boldsymbol{u}}^{\varepsilon, \ell}-\tilde{\boldsymbol{u}}^{\varepsilon, \ell-1}\right\|^{2}+\frac{\alpha-1}{\alpha}\left\|\boldsymbol{u}^{\varepsilon, \ell}-\tilde{\boldsymbol{u}}^{\varepsilon, \ell}\right\|^{2}+2 v k\left\|\nabla \tilde{\boldsymbol{u}}^{\varepsilon, \ell}\right\|^{2} \\
+2 \varepsilon k\left\|\tilde{\boldsymbol{p}}^{\varepsilon, \ell}\right\|^{2}+\alpha k^{2}\left\|\nabla \phi^{\varepsilon, \ell}\right\|^{2}-\alpha k^{2}\left\|\nabla \phi^{\varepsilon, \ell-1}\right\|^{2} \leq 2\left(\Delta_{\ell} \boldsymbol{W}, \tilde{\boldsymbol{u}}^{\varepsilon, \ell}\right) .
\end{gathered}
$$

Step (II) Case $q=1$. Summing (3.36) from $\ell=1$ to $\ell=m$, we get

$$
\begin{aligned}
\left\|\boldsymbol{u}^{\varepsilon, m}\right\|^{2} & +\sum_{\ell=1}^{m}\left\|\tilde{\boldsymbol{u}}^{\varepsilon, \ell}-\tilde{\boldsymbol{u}}^{\varepsilon, \ell-1}\right\|^{2}+\left(\frac{\alpha-1}{\alpha}\right) \sum_{\ell=1}^{m}\left\|\boldsymbol{u}^{\varepsilon, \ell}-\tilde{\boldsymbol{u}}^{\varepsilon, \ell}\right\|^{2}+2 v k \sum_{\ell=1}^{M}\left\|\nabla \tilde{\boldsymbol{u}}^{\varepsilon, \ell}\right\|^{2} \\
& +2 \varepsilon k \sum_{\ell=1}^{m}\left\|\tilde{\boldsymbol{p}}^{\varepsilon, \ell}\right\|^{2}+\alpha k^{2}\left\|\nabla \phi^{\varepsilon, m}\right\|^{2} \leq\left\|\boldsymbol{u}^{0}\right\|^{2}+2 \sum_{\ell=1}^{m}\left(\Delta_{\ell} \boldsymbol{W}, \tilde{\boldsymbol{u}}^{\varepsilon, \ell}\right) .
\end{aligned}
$$

The last term of the right side can be splitted as follows,

$$
\operatorname{Noise}_{1}^{\varepsilon, m}:=2 \sum_{\ell=1}^{m}\left(\Delta_{\ell} \boldsymbol{W}, \tilde{\boldsymbol{u}}^{\varepsilon, \ell}\right)=2 \sum_{\ell=1}^{m}\left(\Delta_{\ell} \boldsymbol{W}, \tilde{\boldsymbol{u}}^{\varepsilon, \ell}-\tilde{\boldsymbol{u}}^{\varepsilon, \ell-1}\right)+2 \sum_{\ell=1}^{m}\left(\Delta_{\ell} \boldsymbol{W}, \tilde{\boldsymbol{u}}^{\varepsilon, \ell-1}\right) .
$$

Let $\delta_{1}>0$ be an arbitrary number. Applying the Young inequality to the first term on the right side, we get

$$
\ldots \leq C\left(\delta_{1}\right) \sum_{\ell=1}^{m}\left\|\Delta_{\ell} \boldsymbol{W}\right\|^{2}+\delta_{1} \sum_{\ell=1}^{m}\left\|\tilde{\boldsymbol{u}}^{\varepsilon, \ell}-\tilde{\boldsymbol{u}}^{\varepsilon, \ell-1}\right\|^{2}+2 \sum_{\ell=1}^{m}\left(\Delta_{\ell} \boldsymbol{W}, \tilde{\boldsymbol{u}}^{\varepsilon, \ell-1}\right)
$$


Taking first the maximum of (3.38) over $1 \leq m \leq M$, and then the expectation, exactly with this order, give the following estimate

$$
\begin{aligned}
\mathbb{E} \max _{1 \leq m \leq M} \operatorname{Noise}_{1}^{\varepsilon, m} \leq C\left(\delta_{1}\right) \sum_{\ell=1}^{M} \mathbb{E}\left\|\Delta_{\ell} \boldsymbol{W}\right\|^{2} & +\delta_{1} \sum_{\ell=1}^{M} \mathbb{E}\left\|\tilde{\boldsymbol{u}}^{\varepsilon, \ell}-\tilde{\boldsymbol{u}}^{\varepsilon, \ell-1}\right\|^{2} \\
& +2 \mathbb{E} \max _{1 \leq m \leq M} \sum_{\ell=1}^{m}\left(\Delta_{\ell} \boldsymbol{W}, \tilde{\boldsymbol{u}}^{\varepsilon, \ell-1}\right) .
\end{aligned}
$$

It follows from (2.3), that $\mathbb{E}\left\|\Delta_{\ell} W\right\|^{2}=k$. By applying successively the Burkholder-David-Gundy inequality, the Hölder inequality, and the Young inequality to the last term of (3.39), we obtain

$$
\begin{aligned}
\mathbb{E}_{1 \leq m \leq M} \operatorname{mox}_{1 \leq \mathrm{N}_{1}^{\varepsilon, m}} & \leq C\left(\delta_{1}, T\right)+\delta_{1} \sum_{\ell=1}^{M} \mathbb{E}\left\|\boldsymbol{u}^{\varepsilon, \ell}-\boldsymbol{u}^{\varepsilon, \ell-1}\right\|^{2}+\mathbb{E}\left(\sum_{\ell=1}^{M} k\left\|\boldsymbol{u}^{\varepsilon, \ell-1}\right\|^{2}\right)^{1 / 2} \\
& \leq C\left(\delta_{1}, T, \boldsymbol{u}^{0}\right)+\delta_{1} \sum_{\ell=1}^{M} \mathbb{E}\left\|\boldsymbol{u}^{\varepsilon, \ell}-\boldsymbol{u}^{\varepsilon, \ell-1}\right\|^{2}+\delta_{1} \mathbb{E} \max _{1 \leq \ell \leq M}\left\|\boldsymbol{u}^{\varepsilon, \ell}\right\|^{2}
\end{aligned}
$$

Now, taking the maximum of (3.37) over $1 \leq m \leq M$, and, then, expectation, give the following estimate,

$$
\begin{aligned}
& \mathbb{E} \max _{1 \leq m \leq M}\left\{\left\|\boldsymbol{u}^{\varepsilon, m}\right\|^{2}+\alpha k^{2}\left\|\nabla \phi^{\varepsilon, m}\right\|^{2}\right\} \\
& +\mathbb{E} \sum_{\ell=1}^{m}\left\|\tilde{\boldsymbol{u}}^{\varepsilon, \ell}-\tilde{\boldsymbol{u}}^{\varepsilon, \ell-1}\right\|^{2}+\left(\frac{\alpha-1}{\alpha}\right) \mathbb{E} \sum_{\ell=1}^{m}\left\|\boldsymbol{u}^{\varepsilon, \ell}-\tilde{\boldsymbol{u}}^{\varepsilon, \ell}\right\|^{2} \\
& +v \mathbb{E}\left(k \sum_{\ell=1}^{M}\left\|\nabla \tilde{\boldsymbol{u}}^{\varepsilon, \ell}\right\|^{2}\right)+\varepsilon \mathbb{E}\left(k \sum_{\ell=1}^{m}\left\|\tilde{\boldsymbol{p}}^{\varepsilon, \ell}\right\|^{2}\right) \leq\left\|\boldsymbol{u}^{0}\right\|^{2}+\mathbb{E} \max _{1 \leq m \leq M} \text { Noise }_{1}^{\varepsilon, m} .
\end{aligned}
$$

The terms with $\left\|\tilde{\boldsymbol{u}}^{\varepsilon, \ell}-\tilde{\boldsymbol{u}}^{\varepsilon, \ell-1}\right\|^{2}$ and $\max _{1 \leq \ell \leq M}\left\|\tilde{\boldsymbol{u}}^{\varepsilon, \ell}\right\|^{2}$ of (3.39) are absorbed by the left hand side of (3.40) which leads to

$$
\begin{aligned}
& \left(1-\delta_{1}\right) \mathbb{E} \max _{1 \leq m \leq M}\left\|\boldsymbol{u}^{\varepsilon, m}\right\|^{2}+\alpha k^{2} \mathbb{E} \max _{1 \leq m \leq M}\left\|\nabla \phi^{\varepsilon, m}\right\|^{2} \\
& +\left(1-\delta_{1}\right) \mathbb{E} \sum_{\ell=1}^{m}\left\|\tilde{\boldsymbol{u}}^{\varepsilon, \ell}-\tilde{\boldsymbol{u}}^{\varepsilon, \ell-1}\right\|^{2}+\left(\frac{\alpha-1}{\alpha}\right) \mathbb{E} \sum_{\ell=1}^{m}\left\|\boldsymbol{u}^{\varepsilon, \ell}-\tilde{\boldsymbol{u}}^{\varepsilon, \ell}\right\|^{2} \\
& +v \mathbb{E}\left(k \sum_{\ell=1}^{M}\left\|\nabla \tilde{\boldsymbol{u}}^{\varepsilon, \ell}\right\|^{2}\right)+\varepsilon \mathbb{E}\left(k \sum_{\ell=1}^{m}\left\|\tilde{\boldsymbol{p}}^{\varepsilon, \ell}\right\|^{2}\right) \leq C\left(\delta_{1}, T, \boldsymbol{u}^{0}\right) .
\end{aligned}
$$

The parameters $\alpha$ and $\delta_{1}$ are chosen such that the left hand side stays positive. Thus, we chose $\alpha>1$ and $0<\delta_{1}<1$.

Step (III) Case $q=2$. We multiply (3.36) by $2\left\|\boldsymbol{u}^{\varepsilon, \ell}\right\|^{2}$ and use again the algebraic identity (3.31) to give

$$
\begin{aligned}
& \left\|\boldsymbol{u}^{\varepsilon, \ell}\right\|^{4}-\left\|\boldsymbol{u}^{\varepsilon, \ell-1}\right\|^{4}+2\left\|\tilde{\boldsymbol{u}}^{\varepsilon, \ell}-\tilde{\boldsymbol{u}}^{\varepsilon, \ell-1}\right\|^{2}\left\|\boldsymbol{u}^{\varepsilon, \ell}\right\|^{2} \\
& +\frac{\alpha-1}{\alpha}\left\|\boldsymbol{u}^{\varepsilon, \ell}-\tilde{\boldsymbol{u}}^{\varepsilon, \ell}\right\|^{2}\left\|\boldsymbol{u}^{\varepsilon, \ell}\right\|^{2}+4 v k\left\|\nabla \tilde{\boldsymbol{u}}^{\varepsilon, \ell}\right\|^{2}\left\|\boldsymbol{u}^{\varepsilon, \ell}\right\|^{2}+4 \varepsilon k\left\|\tilde{\mathfrak{p}}^{\varepsilon, \ell}\right\|^{2}\left\|\boldsymbol{u}^{\varepsilon, \ell}\right\|^{2} \\
& +2 \alpha k^{2}\left\|\nabla \phi^{\varepsilon, \ell}\right\|^{2}\left\|\boldsymbol{u}^{\varepsilon, \ell}\right\|^{2}-2 \alpha k^{2}\left\|\nabla \phi^{\varepsilon, \ell-1}\right\|^{2}\left\|\boldsymbol{u}^{\varepsilon, \ell}\right\|^{2}=2\left(\Delta_{\ell} \boldsymbol{W}, \tilde{\boldsymbol{u}}^{\varepsilon, \ell}\right)\left\|\boldsymbol{u}^{\varepsilon, \ell}\right\|^{2} .
\end{aligned}
$$


On the left hand side we use the same calculation that we used on the term Noise ${ }_{1}^{\varepsilon, m}$. In particular, we compute

$$
\begin{aligned}
\operatorname{Noise}_{2}^{\varepsilon, m}:= & 2 \sum_{\ell=1}^{m}\left(\Delta_{\ell} \boldsymbol{W}, \tilde{\boldsymbol{u}}^{\varepsilon, \ell}\right)\left\|\boldsymbol{u}^{\varepsilon, \ell}\right\|^{2} \\
\leq C\left(\delta_{1}\right) \sum_{\ell=1}^{m}\left\|\Delta_{\ell} \boldsymbol{W}\right\|^{2}+ & \delta_{1} \sum_{\ell=1}^{m}\left\|\tilde{\boldsymbol{u}}^{\varepsilon, \ell}-\tilde{\boldsymbol{u}}^{\varepsilon, \ell-1}\right\|^{2}\left\|\boldsymbol{u}^{\varepsilon, \ell}\right\|^{2} \\
& +2 \sum_{\ell=1}^{m}\left(\Delta_{\ell} \boldsymbol{W}, \tilde{\boldsymbol{u}}^{\varepsilon, \ell-1}\right)\left\|\boldsymbol{u}^{\varepsilon, \ell}\right\|^{2} .
\end{aligned}
$$

In the next step, we first take the maximum of (3.43) over $1 \leq m \leq M$, and, then, we take the expectation, exactly with this order. Now, applying the Young inequality, and the Hölder inequality, and using (3.41) to bound some terms, we can find a constant $C=C\left(\delta_{1}, \delta_{2}, L, T, \boldsymbol{u}^{0}, v\right)>0$ such that

$$
\mathbb{E} \max _{1 \leq m \leq M} \operatorname{Noise}_{2}^{\varepsilon, m} \leq C+\delta_{1} \sum_{\ell=1}^{M}\left\|\tilde{\boldsymbol{u}}^{\varepsilon, \ell}-\tilde{\boldsymbol{u}}^{\varepsilon, \ell-1}\right\|^{2}\left\|\boldsymbol{u}^{\varepsilon, \ell}\right\|^{2}+\delta_{2} \mathbb{E} \max _{1 \leq \ell \leq M}\left\|\boldsymbol{u}^{\varepsilon, \ell}\right\|^{4}
$$

Summing up (3.44) for $\ell=1$ to $\ell=m$, taking the maximum over $1 \leq m \leq M$, and taking the expectation in (3.42) we have

$$
\begin{aligned}
& \mathbb{E} \max _{1 \leq m \leq M}\left\{\left\|\boldsymbol{u}^{\varepsilon, m}\right\|^{4}+\alpha k^{2}\left\|\nabla \phi^{\varepsilon, \ell}\right\|^{2}\left\|\boldsymbol{u}^{\varepsilon, \ell}\right\|^{2}\right\}+\mathbb{E} \sum_{\ell=1}^{M}\left\|\tilde{\boldsymbol{u}}^{\varepsilon, \ell}-\tilde{\boldsymbol{u}}^{\varepsilon, \ell-1}\right\|^{2}\left\|\boldsymbol{u}^{\varepsilon, \ell}\right\|^{2} \\
& +\left(\frac{\alpha-1}{\alpha}\right) \mathbb{E} \sum_{\ell=1}^{M}\left\|\boldsymbol{u}^{\varepsilon, \ell}-\tilde{\boldsymbol{u}}^{\varepsilon, \ell}\right\|^{2}\left\|\boldsymbol{u}^{\varepsilon, \ell}\right\|^{2}+v \mathbb{E}\left(k \sum_{\ell=1}^{M}\left\|\nabla \tilde{\boldsymbol{u}}^{\varepsilon, \ell}\right\|^{2}\left\|\boldsymbol{u}^{\varepsilon, \ell}\right\|^{2}\right) \\
& +\varepsilon \mathbb{E}\left(k \sum_{\ell=1}^{M}\left\|\tilde{\boldsymbol{p}}^{\varepsilon, \ell}\right\|^{2}\left\|\boldsymbol{u}^{\varepsilon, \ell}\right\|^{2}\right) \\
& \leq C\left(\delta_{1}, \delta_{2}, L, T, \boldsymbol{u}^{0}, v\right)+\delta_{1} \sum_{\ell=1}^{M}\left\|\tilde{\boldsymbol{u}}^{\varepsilon, \ell}-\tilde{\boldsymbol{u}}^{\varepsilon, \ell-1}\right\|^{2}\left\|\boldsymbol{u}^{\varepsilon, \ell}\right\|^{2}+\delta_{2} \mathbb{E} \max _{1 \leq \ell \leq M}\left\|\boldsymbol{u}^{\varepsilon, \ell}\right\|^{4} .
\end{aligned}
$$

The terms with $\left\|\boldsymbol{u}^{\varepsilon, \ell}\right\|^{4}$ and $\left\|\tilde{\boldsymbol{u}}^{\varepsilon, \ell}-\tilde{\boldsymbol{u}}^{\varepsilon, \ell-1}\right\|^{2}\left\|\boldsymbol{u}^{\varepsilon, \ell}\right\|^{2}$ are absorbed by the left side of (3.45). Therefore, we get

$$
\begin{aligned}
& \mathbb{E} \max _{1 \leq m \leq M}\left\{\left(1-\delta_{2}\right)\left\|\boldsymbol{u}^{\varepsilon, m}\right\|^{4}+\alpha k^{2}\left\|\nabla \phi^{\varepsilon, m}\right\|^{2}\left\|\boldsymbol{u}^{\varepsilon, m}\right\|^{2}\right\} \\
& +\left(1-\delta_{1}\right) \mathbb{E} \sum_{\ell=1}^{M}\left\|\tilde{\boldsymbol{u}}^{\varepsilon, \ell}-\tilde{\boldsymbol{u}}^{\varepsilon, \ell-1}\right\|^{2}\left\|\boldsymbol{u}^{\varepsilon, \ell}\right\|^{2}+\left(\frac{\alpha-1}{\alpha}\right) \mathbb{E} \sum_{\ell=1}^{M}\left\|\boldsymbol{u}^{\varepsilon, \ell}-\tilde{\boldsymbol{u}}^{\varepsilon, \ell}\right\|^{2}\left\|\boldsymbol{u}^{\varepsilon, \ell}\right\|^{2} \\
& +v \mathbb{E}\left(k \sum_{\ell=1}^{M}\left\|\nabla \tilde{\boldsymbol{u}}^{\varepsilon, \ell}\right\|^{2}\left\|\boldsymbol{u}^{\varepsilon, \ell}\right\|^{2}\right)+\varepsilon \mathbb{E}\left(k \sum_{\ell=1}^{M}\left\|\tilde{\boldsymbol{p}}^{\varepsilon, \ell}\right\|^{2}\left\|\boldsymbol{u}^{\varepsilon, \ell}\right\|^{2}\right) \leq C\left(\delta_{1}, \delta_{2}, L, T, \boldsymbol{u}^{0}, v\right) .
\end{aligned}
$$

We conclude by choosing $\alpha, \delta_{1}$, and $\delta_{2}$ such that, $(\alpha-1)>0,\left(1-\delta_{1}\right)>0$, and $\left(1-\delta_{2}\right)>0$.

In the next lemma we use the LBB inequality (see $[1,8]$ )

$$
\|\mathrm{p}\| \leq C \sup _{\boldsymbol{\varphi} \in \mathbb{W}^{1,2}} \frac{(\nabla \mathrm{p}, \boldsymbol{\varphi})}{\|\boldsymbol{\varphi}\|_{1}}
$$

to transfer the estimate from the velocity fields $\boldsymbol{u}^{\varepsilon, \ell}$ to the pressure fields $\mathrm{p}^{\varepsilon, \ell}$.

We start with a direct discretization of (1.1) which leads to the following algorithm: 
Algorithm 2. Assume $\boldsymbol{u}^{0}:=\boldsymbol{u}_{0}$ with $\left\|\boldsymbol{u}^{0}\right\| \leq C$. Find for every $\ell \in\{1, \ldots, M\}$ a pair of random variables $\left(\boldsymbol{u}^{\ell}, \mathrm{p}^{\ell}\right)$ with values in $\mathbb{V} \times L_{\text {per }}^{2}$, such that $\mathbb{P}$-a.s.

$$
\begin{aligned}
\left(\boldsymbol{u}^{\ell}-\boldsymbol{u}^{\ell-1}, \boldsymbol{\varphi}\right)+v k\left(\nabla \boldsymbol{u}^{\ell}, \nabla \boldsymbol{\varphi}\right)+k \tilde{b}\left(\boldsymbol{u}^{\ell}, \boldsymbol{u}^{\ell}, \boldsymbol{\varphi}\right) & \\
+k\left(\nabla \mathrm{p}^{\ell}, \boldsymbol{\varphi}\right) & =\left(\Delta_{\ell} \boldsymbol{W}, \boldsymbol{\varphi}\right), \quad \forall \boldsymbol{\varphi} \in \mathbb{W}_{\text {per }}^{1,2} \\
\left(\operatorname{div} \boldsymbol{u}^{\ell}, \chi\right) & =0, \quad \forall \chi \in L_{\text {per }}^{2} .
\end{aligned}
$$

We define the sequences of errors $\mathrm{E}^{\ell}=\boldsymbol{u}^{\ell}-\boldsymbol{u}^{\varepsilon, \ell}, \tilde{\mathrm{E}}^{\ell}=\boldsymbol{u}^{\ell}-\tilde{\boldsymbol{u}}^{\varepsilon, \ell}$, and $\mathrm{Q}^{\ell}=\mathrm{p}^{\ell}-\mathrm{p}^{\varepsilon, \ell}$. We subtract (3.25) and (3.26) from (3.47) and (3.48), and get

$$
\begin{aligned}
\left(\mathrm{E}^{\ell}-\mathrm{E}^{\ell-1}, \boldsymbol{\varphi}\right) & +v k\left(\nabla \tilde{\mathrm{E}}^{\ell}, \nabla \boldsymbol{\varphi}\right) \\
& +k\left(\nabla \mathrm{Q}^{\ell}, \boldsymbol{\varphi}\right)=k \tilde{b}\left(\tilde{\boldsymbol{u}}^{\varepsilon, \ell-1}, \tilde{\boldsymbol{u}}^{\varepsilon, \ell}, \boldsymbol{\varphi}\right)-k \tilde{b}\left(\boldsymbol{u}^{\ell}, \boldsymbol{u}^{\ell}, \boldsymbol{\varphi}\right), \forall \boldsymbol{\varphi} \in \mathbb{W}_{\text {per }}^{1,2} .
\end{aligned}
$$

Lemma 3.3. Under the assumption of Lemma 3.2, there exists a constant $C=C\left(L, T, \boldsymbol{u}_{0}\right)>0$ such that for every $\varepsilon>0$, the iterates $\left\{\mathrm{p}^{\varepsilon, \ell}: 1 \leq \ell \leq M\right\}$ solving Algorithm 1 satisfies

$$
\mathbb{E}\left(k \sum_{\ell=1}^{M}\left\|\mathrm{p}^{\varepsilon, \ell}\right\|^{2}\right) \leq C .
$$

Proof. Since $\left(\mathrm{E}^{\ell}-\mathrm{E}^{\ell-1}\right) \in \mathcal{D}\left(\boldsymbol{A}^{-1}\right)$, we can take $\boldsymbol{\varphi}=\boldsymbol{A}^{-1}\left(\mathrm{E}^{\ell}-\mathrm{E}^{\ell-1}\right)$ in (3.49) and use Proposition 2.1. Then we apply the Young inequality, and use estimate (2.11) of $\tilde{b}$. This leads to the following results:

i) $c_{2}\left\|\mathrm{E}^{\ell}-\mathrm{E}^{\ell-1}\right\|_{-1}^{2} \leq\left(\mathrm{E}^{\ell}-\mathrm{E}^{\ell-1}, A^{-1}\left(\mathrm{E}^{\ell}-\mathrm{E}^{\ell-1}\right)\right)$,

ii) $v k\left(\nabla \mathrm{E}^{\ell}, \nabla A^{-1}\left(\mathrm{E}^{\ell}-\mathrm{E}^{\ell-1}\right)\right) \leq C\left(\delta_{1}\right) v^{2} k^{2}\left\|\mathrm{E}^{\ell}\right\|_{1}^{2}+\delta_{1}\left\|\mathrm{E}^{\ell}-\mathrm{E}^{\ell-1}\right\|_{-1^{\prime}}^{2}$

iii) $k\left(\nabla \mathrm{Q}^{\ell}, A^{-1}\left(\mathrm{E}^{\ell}-\mathrm{E}^{\ell-1}\right)\right)=0$,

iv) $k \tilde{b}\left(\tilde{\boldsymbol{u}}^{\varepsilon, \ell-1}, \tilde{\boldsymbol{u}}^{\varepsilon, \ell}, \boldsymbol{A}^{-1}\left(\mathrm{E}^{\ell}-\mathrm{E}^{\ell-1}\right)\right) \leq C\left(L, \delta_{1}\right) \frac{k^{2}}{2}\left\|\tilde{\boldsymbol{u}}^{\varepsilon, \ell-1}\right\|^{2}\left\|\tilde{\boldsymbol{u}}^{\varepsilon, \ell-1}\right\|_{1}^{2}+C\left(L, \delta_{1}\right) \frac{k^{2}}{2}\left\|\tilde{\boldsymbol{u}}^{\varepsilon, \ell}\right\|^{2}\left\|\tilde{\boldsymbol{u}}^{\varepsilon, \ell}\right\|_{1}^{2}+\delta_{1} \| \mathrm{E}^{\ell}-$ $\mathrm{E}^{\ell-1} \|_{-1}^{2}$

v) $k \tilde{b}\left(\boldsymbol{u}^{\ell}, \boldsymbol{u}^{\ell}, \boldsymbol{A}^{-1}\left(\mathrm{E}^{\ell}-\mathrm{E}^{\ell-1}\right)\right) \leq C\left(L, \delta_{1}\right) k^{2}\left\|\boldsymbol{u}^{\ell}\right\|^{2}\left\|\boldsymbol{u}^{\ell}\right\|_{1}^{2}+\delta_{1}\left\|\mathrm{E}^{\ell}-\mathrm{E}^{\ell-1}\right\|_{-1}^{2}$.

Fixing $\delta_{1}>0$ such that $\left(c_{2}-3 \delta_{1}\right)>0$, and collecting $\left.\left.\left.\left.i\right), i i\right), i i i\right), i v\right)$, and $\left.v\right)$, we obtain

$$
\begin{aligned}
& \left(c_{2}-3 \delta_{1}\right) \mathbb{E} \sum_{\ell=1}^{M}\left\|\mathrm{E}^{\ell}-\mathrm{E}^{\ell-1}\right\|_{-1}^{2} \leq C\left(L, \delta_{1}, v\right) k \mathbb{E}\left(k \sum_{\ell=1}^{M}\left\|\boldsymbol{u}^{\varepsilon, \ell}\right\|_{1}^{2}\right)+k \mathbb{E}\left(k \sum_{\ell=1}^{M}\left\|\boldsymbol{u}^{\ell}\right\|_{1}^{2}\right) \\
& +k \mathbb{E}\left(k \sum_{\ell=1}^{M}\left\|\tilde{\boldsymbol{u}}^{\varepsilon, \ell-1}\right\|^{2}\left\|\tilde{\boldsymbol{u}}^{\varepsilon, \ell-1}\right\|_{1}^{2}\right)+k \mathbb{E}\left(k \sum_{\ell=1}^{M}\left\|\tilde{\boldsymbol{u}}^{\varepsilon, \ell}\right\|^{2}\left\|\tilde{\boldsymbol{u}}^{\varepsilon, \ell}\right\|_{1}^{2}\right)+k \mathbb{E}\left(k \sum_{\ell=1}^{M}\left\|\boldsymbol{u}^{\ell}\right\|^{2}\left\|\boldsymbol{u}^{\ell}\right\|_{1}^{2}\right) .
\end{aligned}
$$

By Lemma 3.2 and [9, Lemma 3.1 (iii)] we obtain

$$
\mathbb{E} \sum_{\ell=1}^{M}\left\|\mathrm{E}^{\ell}-\mathrm{E}^{\ell-1}\right\|_{-1}^{2} \leq C\left(T, L, u^{0}\right) k .
$$

Now we rearrange (3.49) and get

$$
k\left(\nabla \mathrm{Q}^{\ell}, \boldsymbol{\varphi}\right)=-\left(\mathrm{E}^{\ell}-\mathrm{E}^{\ell-1}, \boldsymbol{\varphi}\right)-v k\left(\nabla \tilde{\mathrm{E}}^{\ell}, \nabla \boldsymbol{\varphi}\right)+k \tilde{b}\left(\tilde{\boldsymbol{u}}^{\varepsilon, \ell-1}, \tilde{\boldsymbol{u}}^{\varepsilon, \ell}, \boldsymbol{\varphi}\right)-k \tilde{b}\left(\boldsymbol{u}^{\ell}, \boldsymbol{u}^{\ell}, \boldsymbol{\varphi}\right) .
$$

With the skew symmetry property of $\tilde{b}$ (see (2.7)) and the estimate (2.11), identity (3.51) becomes

$$
\begin{aligned}
\frac{k\left(\nabla \mathrm{Q}^{\ell}, \boldsymbol{\varphi}\right)}{\|\boldsymbol{\varphi}\|_{1}} \leq\left\|\mathrm{E}^{\ell}-\mathrm{E}^{\ell-1}\right\|_{-1} & +v k\left\|\nabla \tilde{\mathrm{E}}^{\ell}\right\|+C(L) k\left\|\tilde{\boldsymbol{u}}^{\varepsilon, \ell-1}\right\|\left\|\tilde{\boldsymbol{u}}^{\varepsilon, \ell-1}\right\|_{1} \\
& +C(L) k\left\|\boldsymbol{u}^{\varepsilon, \ell}\right\|\left\|\boldsymbol{u}^{\varepsilon, \ell}\right\|_{1}+C(L) k\left\|\boldsymbol{u}^{\ell}\right\|\left\|\boldsymbol{u}^{\ell}\right\|_{1} .
\end{aligned}
$$


Using the inequality (3.46), we have

$$
\begin{aligned}
k^{2}\left\|\mathrm{Q}^{\ell}\right\|^{2} \leq C\left\|\mathrm{E}^{\ell}-\mathrm{E}^{\ell-1}\right\|_{-1}^{2} & +v^{2} k^{2}\left\|\nabla \tilde{\mathrm{E}}^{\ell}\right\|^{2}+C(L) k^{2}\left\|\tilde{\boldsymbol{u}}^{\varepsilon, \ell-1}\right\|^{2}\left\|\tilde{\boldsymbol{u}}^{\varepsilon, \ell-1}\right\|_{1}^{2} \\
& +C(L) k^{2}\left\|\tilde{\boldsymbol{u}}^{\varepsilon, \ell}\right\|^{2}\left\|\tilde{\boldsymbol{u}}^{\varepsilon, \ell}\right\|_{1}^{2}+C(L) k^{2}\left\|\boldsymbol{u}^{\ell}\right\|^{2}\left\|\boldsymbol{u}^{\ell}\right\|_{1}^{2} .
\end{aligned}
$$

Summing up for $\ell=1$ to $\ell=M$, and taking expectation, we obtain

$$
\begin{aligned}
k \mathbb{E}\left(k \sum_{\ell=1}^{M}\left\|\mathrm{Q}^{\ell}\right\|^{2}\right) \leq & C \mathbb{E} \sum_{\ell=1}^{M}\left\|\mathrm{E}^{\ell}-\mathrm{E}^{\ell-1}\right\|_{-1}^{2}+v^{2} k \mathbb{E}\left(k \sum_{\ell=1}^{M}\left\|\nabla \tilde{\boldsymbol{u}}^{\varepsilon, \ell}\right\|^{2}\right) \\
& +v^{2} k \mathbb{E}\left(k \sum_{\ell=1}^{M}\left\|\nabla \boldsymbol{u}^{\ell}\right\|^{2}\right)+C(L) k \mathbb{E}\left(k \sum_{\ell=1}^{M}\left\|\tilde{\boldsymbol{u}}^{\varepsilon, \ell-1}\right\|^{2}\left\|\tilde{\boldsymbol{u}}^{\varepsilon, \ell-1}\right\|_{1}^{2}\right) \\
& +C(L) k \mathbb{E}\left(k \sum_{\ell=1}^{M}\left\|\tilde{\boldsymbol{u}}^{\varepsilon, \ell}\right\|^{2}\left\|\tilde{\boldsymbol{u}}^{\varepsilon, \ell}\right\|_{1}^{2}\right)+C(L) k \mathbb{E}\left(k \sum_{\ell=1}^{M}\left\|\boldsymbol{u}^{\ell}\right\|^{2}\left\|\boldsymbol{u}^{\ell}\right\|_{1}^{2}\right) .
\end{aligned}
$$

From Lemma 3.2, [9, Lemma 3.1 (iii)], and estimate (3.50), we obtain

$$
\mathbb{E}\left(k \sum_{\ell=1}^{M}\left\|\mathrm{Q}^{\ell}\right\|^{2}\right) \leq C\left(T, L, v, \boldsymbol{u}^{0}\right)
$$

The Minkowsky inequality and Poincaré inequality imply

$$
\mathbb{E}\left(k \sum_{\ell=1}^{M}\left\|\mathrm{p}^{\varepsilon, \ell}\right\|^{2}\right) \leq C\left(T, L, v, \boldsymbol{u}^{0}\right)+C(L) \mathbb{E}\left(k \sum_{\ell=1}^{M}\left\|\nabla \mathrm{p}^{\ell}\right\|^{2}\right) .
$$

We finish the proof with using [13, Lemma 3.2 (i)], where the authors proved that

$$
\mathbb{E}\left(k \sum_{\ell=1}^{M}\left\|\nabla \mathrm{p}^{\ell}\right\|^{2}\right) \leq C(T)
$$

3.2. Auxiliary error estimates. We start with Algorithm 3. Let $z=\left\{z(t, \cdot): t \in\left[t_{\ell-1}, t_{\ell}\right]\right\}$ be the strong solution of (2.19) as defined in Definition 2.2 and $\pi=\left\{\pi(t, \cdot): t \in\left[t_{\ell-1}, t_{\ell}\right]\right\}$ the associated pressure, i.e. for every $t \in\left[t_{\ell-1}, t_{\ell}\right]$, all $\varphi \in \mathbb{W}^{1,2}$, and all $\chi \in L_{\text {per }}^{2}$, we have $\mathbb{P}$-a.s.

$$
\begin{aligned}
\left(z\left(t_{\ell}\right)-z\left(t_{\ell-1}\right), \boldsymbol{\varphi}\right)+ & v \int_{t_{\ell-1}}^{t_{\ell}}(\nabla z(s), \nabla \boldsymbol{\varphi}) d s \\
& +\int_{t_{\ell-1}}^{t_{\ell}}(\nabla \pi(s), \boldsymbol{\varphi}) d s=\int_{t_{\ell-1}}^{t_{\ell}}(\boldsymbol{\varphi}, d \boldsymbol{W}(s)), \\
& \left(\operatorname{div} \boldsymbol{z}\left(t_{\ell}\right), \chi\right)=0 .
\end{aligned}
$$

For (3.52) and (3.53) we have the following algorithm:

Algorithm 3 (First auxiliary algorithm). Let $z^{0}:=0$. Find for every $\ell \in\{1, \ldots, M\}$ a pair of random variables $\left(\boldsymbol{z}^{\ell}, \pi^{\ell}\right)$ with values in $\mathbb{W}_{\text {per }}^{1,2} \times L_{\text {per, }}^{2}$ such that we have $\mathbb{P}$-a.s.

- Penalization:

$$
\begin{aligned}
\left(\tilde{z}^{\varepsilon, \ell}-z^{\varepsilon, \ell-1}, \boldsymbol{\varphi}\right)+v k\left(\nabla \tilde{z}^{\varepsilon, \ell}, \nabla \boldsymbol{\varphi}\right)+k\left(\nabla \tilde{\pi}^{\varepsilon, \ell}, \boldsymbol{\varphi}\right) & \\
+k\left(\nabla \tilde{\xi}^{\varepsilon, \ell-1}, \boldsymbol{\varphi}\right) & =\left(\Delta_{\ell} \boldsymbol{W}, \boldsymbol{\varphi}\right), \quad \forall \boldsymbol{\varphi} \in \mathbb{W}_{\text {per }}^{1,2}, \\
\left(\operatorname{div} \tilde{z}^{\varepsilon, \ell}, \chi\right)+\varepsilon\left(\tilde{\pi}^{\varepsilon, \ell}, \chi\right) & =0, \quad \forall \chi \in L_{\text {per }}^{2}
\end{aligned}
$$

- Projection:

$$
\begin{aligned}
\left(\boldsymbol{z}^{\varepsilon, \ell}-\tilde{z}^{\varepsilon, \ell}, \boldsymbol{\varphi}\right)+\alpha k\left(\nabla\left(\xi^{\varepsilon, \ell}-\xi^{\varepsilon, \ell-1}\right), \boldsymbol{\varphi}\right) & =0, \forall \boldsymbol{\varphi} \in \mathbb{W}_{\text {per }}^{1,2} \\
\left(\operatorname{div} z^{\varepsilon, \ell}, \chi\right) & =0, \forall \chi \in L_{\text {per }}^{2} \\
\pi^{\varepsilon, \ell} & =\tilde{\pi}^{\varepsilon, \ell}+\xi^{\varepsilon, \ell}+\alpha\left(\xi^{\varepsilon, \ell}-\xi^{\varepsilon, \ell-1}\right) .
\end{aligned}
$$


Define the errors $\tilde{\boldsymbol{\epsilon}}^{\ell}=\boldsymbol{z}\left(t_{\ell}\right)-\tilde{\boldsymbol{z}}^{\varepsilon, \ell}, \boldsymbol{\epsilon}^{\ell}=\boldsymbol{z}\left(t_{\ell}\right)-\boldsymbol{z}^{\varepsilon, \ell}$ and $\boldsymbol{\omega}^{\ell}=\pi\left(t_{\ell}\right)-\pi^{\varepsilon, \ell}$. We subtract (3.54) from (3.52) to get

$$
\begin{aligned}
\left(\tilde{\boldsymbol{\epsilon}}^{\ell}-\boldsymbol{\epsilon}^{\ell-1}, \boldsymbol{\varphi}\right) & +v \int_{t_{\ell-1}}^{t_{\ell}}\left(\nabla\left(z(s)-\tilde{z}^{\varepsilon, \ell}\right), \nabla \boldsymbol{\varphi}\right) d s \\
& +\int_{t_{\ell-1}}^{t_{\ell}}\left(\nabla\left(\pi(s)-\tilde{\pi}^{\varepsilon, \ell}-\xi^{\varepsilon, \ell-1}\right), \boldsymbol{\varphi}\right) d s=0,
\end{aligned}
$$

and choose $\chi=\operatorname{div} \varphi$ in (3.55) to get

$$
-\left(\nabla \pi^{\varepsilon, \ell}, \boldsymbol{\varphi}\right)=\frac{1}{\varepsilon}\left(\nabla \operatorname{div} \tilde{z}^{\varepsilon, \ell}, \boldsymbol{\varphi}\right) .
$$

Thanks to the following identities

$$
\begin{gathered}
\left(\nabla\left(z(s)-\tilde{z}^{\varepsilon, \ell}\right), \nabla \boldsymbol{\varphi}\right)=\left(\nabla \tilde{\boldsymbol{\epsilon}}^{\ell}, \nabla \boldsymbol{\varphi}\right)+\left(\nabla\left(z(s)-z\left(t_{\ell}\right)\right), \nabla \boldsymbol{\varphi}\right) \text { and } \\
\left(\nabla\left(\pi(s)-\pi^{\varepsilon, \ell}-\xi^{\varepsilon, \ell-1}\right), \boldsymbol{\varphi}\right)=(\nabla \pi(s), \boldsymbol{\varphi})+\frac{1}{\varepsilon}\left(\nabla \operatorname{div} \tilde{z}^{\varepsilon, \ell}, \boldsymbol{\varphi}\right)-\left(\nabla \xi^{\varepsilon, \ell-1}, \boldsymbol{\varphi}\right),
\end{gathered}
$$

the equation (3.58) is reduced to

$$
\begin{aligned}
\left(\tilde{\boldsymbol{\epsilon}}^{\ell}-\boldsymbol{\epsilon}^{\ell-1}, \boldsymbol{\varphi}\right)+v k\left(\nabla \tilde{\boldsymbol{\epsilon}}^{\ell}, \nabla \boldsymbol{\varphi}\right) & -\frac{k}{\varepsilon}\left(\nabla \operatorname{div} \tilde{z}^{\varepsilon, \ell}, \boldsymbol{\varphi}\right) \\
& -k\left(\nabla \xi^{\varepsilon, \ell-1}, \boldsymbol{\varphi}\right)=R_{\ell}^{z}(\boldsymbol{\varphi})-\int_{t_{\ell-1}}^{t_{\ell}}(\nabla \pi(s), \boldsymbol{\varphi}) d s,
\end{aligned}
$$

where

$$
R_{\ell}^{z}(\boldsymbol{\varphi})=v \int_{t_{\ell-1}}^{t_{\ell}}\left(\nabla\left(z\left(t_{\ell}\right)-z(s)\right), \nabla \boldsymbol{\varphi}\right) d s .
$$

To (3.62) we associate the following projection equation

$$
\left\{\begin{aligned}
\left(\boldsymbol{\epsilon}^{\ell}-\tilde{\boldsymbol{\epsilon}}^{\ell}, \boldsymbol{\varphi}\right) & =k \alpha\left(\nabla\left(\xi^{\varepsilon, \ell}-\xi^{\varepsilon, \ell-1}\right), \boldsymbol{\varphi}\right), \\
\operatorname{div} \boldsymbol{\epsilon}^{\ell} & =0 .
\end{aligned}\right.
$$

Lemma 3.4. Let $\alpha>1$ and $0<\eta<1 / 2$. For every $\varepsilon>0$, there exists a constant $C=C(T, v, \eta)>0$ such that

$$
\mathbb{E} \max _{1<m \leq M}\left\|\boldsymbol{\epsilon}^{m}\right\|^{2}+v \mathbb{E}\left(k \sum_{\ell=1}^{M}\left\|\nabla \boldsymbol{\epsilon}^{\ell}\right\|^{2}\right) \leq C\left(k^{\eta}+\varepsilon\right) .
$$

Proof. We take $\boldsymbol{\varphi}=2 \tilde{\boldsymbol{\epsilon}}^{\ell}$ in (3.62). Then we use the algebraic identity (3.31) and the fact that $\operatorname{div} \boldsymbol{z}\left(t_{\ell}\right)=0$ to get

$$
\begin{aligned}
\left\|\tilde{\boldsymbol{e}}^{\ell}\right\|^{2}-\left\|\boldsymbol{\epsilon}^{\ell-1}\right\|^{2} & +\left\|\tilde{\boldsymbol{\epsilon}}^{\ell}-\boldsymbol{\epsilon}^{\ell-1}\right\|^{2}+2 v k\left\|\nabla \tilde{\boldsymbol{\epsilon}}^{\ell}\right\|^{2}+\frac{2 k}{\varepsilon}\left\|\operatorname{div} \tilde{\boldsymbol{\epsilon}}^{\ell}\right\|^{2} \\
& =2 k\left(\nabla \xi^{\varepsilon, \ell-1}, \tilde{\boldsymbol{\epsilon}}^{\ell}\right)+R_{\ell}^{z}\left(2 \tilde{\boldsymbol{\epsilon}}^{\ell}\right)+\int_{t_{\ell-1}}^{t_{\ell}}\left(\operatorname{div} \tilde{\boldsymbol{\epsilon}}^{\ell}, \pi(s)\right) d s .
\end{aligned}
$$

Let us take $\boldsymbol{\varphi}=\tilde{\boldsymbol{\epsilon}}^{\ell}+\boldsymbol{\epsilon}^{\ell}$ in (3.63) to get

$$
\begin{aligned}
\frac{\alpha-1}{\alpha}\left(\left\|\boldsymbol{\epsilon}^{\ell}\right\|^{2}-\left\|\tilde{\boldsymbol{\epsilon}}^{\ell}\right\|^{2}+\left\|\tilde{\boldsymbol{\epsilon}}^{\ell}-\boldsymbol{\epsilon}^{\ell}\right\|^{2}\right) & =0, \\
\frac{1}{\alpha}\left(\left\|\boldsymbol{\epsilon}^{\ell}\right\|^{2}-\left\|\tilde{\boldsymbol{\epsilon}}^{\ell}\right\|^{2}\right) & =\frac{k}{2}\left(\nabla\left(\xi^{\varepsilon, \ell}-\xi^{\varepsilon, \ell-1}\right), \tilde{\boldsymbol{\epsilon}}^{\ell}\right) .
\end{aligned}
$$

Collecting (3.65) to (3.67) together, we arrive at

$$
\begin{aligned}
& \left\|\boldsymbol{\epsilon}^{\ell}\right\|^{2}-\left\|\boldsymbol{\epsilon}^{\ell-1}\right\|^{2}+\left\|\tilde{\boldsymbol{\epsilon}}^{\ell}-\boldsymbol{\epsilon}^{\ell-1}\right\|^{2}+\left(\frac{\alpha-1}{\alpha}\right)\left\|\tilde{\boldsymbol{\epsilon}}^{\ell}-\boldsymbol{\epsilon}^{\ell}\right\|^{2}+2 v k\left\|\nabla \tilde{\boldsymbol{\epsilon}}^{\ell}\right\|^{2} \\
& +\frac{2 k}{\varepsilon}\left\|\operatorname{div} \tilde{\boldsymbol{\epsilon}}^{\ell}\right\|^{2} \leq 2 k\left(\nabla\left(\xi^{\varepsilon, \ell}+\xi^{\varepsilon, \ell-1}\right), \tilde{\boldsymbol{\epsilon}}^{\ell}\right)+R_{\ell}^{z}\left(2 \tilde{\boldsymbol{\epsilon}}^{\ell}\right)+\int_{t_{\ell-1}}^{t_{\ell}}\left(\operatorname{div} \tilde{\boldsymbol{\epsilon}}^{\ell}, \pi(s)\right) d s .
\end{aligned}
$$

First, notice that from (3.63) it follows

$$
\tilde{\boldsymbol{\epsilon}}^{\ell}=\boldsymbol{\epsilon}^{\ell}-k \alpha \nabla\left(\xi^{\varepsilon, \ell}-\xi^{\varepsilon, \ell-1}\right) .
$$


Therefore, we have

$$
2 k\left(\nabla\left(\xi^{\varepsilon, \ell}+\xi^{\varepsilon, \ell-1}\right), \tilde{\boldsymbol{\epsilon}}^{\ell}\right)=2 \alpha k^{2}\left\|\xi^{\varepsilon, \ell-1}\right\|^{2}-2 \alpha k^{2}\left\|\xi^{\varepsilon, \ell}\right\|^{2} .
$$

Secondly, applying the Young inequality to $R_{\ell}^{z}\left(2 \tilde{\boldsymbol{\epsilon}}^{\ell}\right)$, we get

$$
R_{\ell}^{z}\left(2 \tilde{\boldsymbol{\epsilon}}^{\ell}\right) \leq C_{\delta_{1}} v \int_{t_{\ell-1}}^{t_{\ell}}\left\|\nabla\left(\boldsymbol{z}\left(t_{\ell}\right)-\boldsymbol{z}(s)\right)\right\|^{2} d s+\delta_{1} k\left\|\nabla \tilde{\boldsymbol{\epsilon}}^{\ell}\right\|^{2},
$$

$$
\int_{t_{\ell-1}}^{t_{\ell}}\left(\operatorname{div} \tilde{\boldsymbol{\epsilon}}^{\ell}, \pi(s)\right) d s \leq \frac{k}{\varepsilon}\left\|\operatorname{div} \tilde{\boldsymbol{\epsilon}}^{\ell}\right\|^{2}+\varepsilon \int_{t_{\ell-1}}^{t_{\ell}}\|\pi(s)\|^{2} d s .
$$

We add (3.69) to (3.71) with (3.68). Summing up for $\ell=1$ to $\ell=m$,

$$
\begin{aligned}
& \left\|\boldsymbol{\epsilon}^{m}\right\|^{2}+2 \alpha k^{2}\left\|\xi^{\varepsilon, m}\right\|^{2}+\left(\frac{\alpha-1}{\alpha}\right) \sum_{\ell=1}^{m}\left\|\tilde{\boldsymbol{\epsilon}}^{\ell}-\boldsymbol{\epsilon}^{\ell}\right\|^{2} \\
& +\left(2-\delta_{1}\right) v\left(k \sum_{\ell=1}^{m}\left\|\nabla \tilde{\boldsymbol{\epsilon}}^{\ell}\right\|^{2}\right)+\frac{1}{\varepsilon} \mathbb{E}\left(k \sum_{\ell=1}^{m}\left\|\operatorname{div} \tilde{\boldsymbol{\epsilon}}^{\ell}\right\|^{2}\right) \\
& \leq C_{\delta_{1}} v \sum_{\ell=1}^{m} \int_{t_{\ell-1}}^{t_{\ell}} \mathbb{E}\left\|\nabla\left(\boldsymbol{z}\left(t_{\ell}\right)-\boldsymbol{z}(s)\right)\right\|^{2}+\varepsilon \sum_{\ell=1}^{m} \int_{t_{\ell-1}}^{t_{\ell}} \mathbb{E}\|\pi(s)\|^{2} d s .
\end{aligned}
$$

Using the identity (2.5), we have

$$
\begin{aligned}
& \frac{1}{\alpha}\left\|\boldsymbol{\epsilon}^{m}\right\|^{2}+\frac{\alpha-1}{\alpha}\left\|\tilde{\boldsymbol{\epsilon}}^{m}\right\|^{2}+2 \alpha k^{2}\left\|\tilde{\zeta}^{\varepsilon, m}\right\|^{2}+\frac{\alpha-1}{\alpha} \sum_{\ell=1}^{m-1}\left\|\tilde{\boldsymbol{\epsilon}}^{\ell}-\boldsymbol{\epsilon}^{\ell}\right\|^{2} \\
& +\left(2-\delta_{1}\right) v\left(k \sum_{\ell=1}^{m}\left\|\nabla \tilde{\boldsymbol{\epsilon}}^{\ell}\right\|^{2}\right)+\frac{1}{\varepsilon} \mathbb{E}\left(k \sum_{\ell=1}^{m}\left\|\operatorname{div} \tilde{\boldsymbol{\epsilon}}^{\ell}\right\|^{2}\right) \\
& \leq C_{\delta_{1}} v \sum_{\ell=1}^{m} \int_{t_{\ell-1}}^{t_{\ell}} \mathbb{E}\left\|\nabla\left(\boldsymbol{z}\left(t_{\ell}\right)-\boldsymbol{z}(s)\right)\right\|^{2}+\varepsilon \sum_{\ell=1}^{m} \int_{t_{\ell-1}}^{t_{\ell}} \mathbb{E}\|\pi(s)\|^{2} d s .
\end{aligned}
$$

Now taking the maximum for $1<m \leq M$, and expectation, we arrive at

$$
\begin{aligned}
& \mathbb{E} \max _{1 \leq m \leq M}\left\{\frac{1}{\alpha}\left\|\boldsymbol{\epsilon}^{m}\right\|^{2}+\frac{\alpha-1}{\alpha}\left\|\tilde{\boldsymbol{\epsilon}}^{m}\right\|^{2}+2 \alpha k^{2}\left\|\tilde{\zeta}^{\varepsilon, m}\right\|^{2}\right\} \\
& +\frac{\alpha-1}{\alpha} \mathbb{E} \sum_{\ell=1}^{M-1}\left\|\tilde{\boldsymbol{\epsilon}}^{\ell}-\boldsymbol{\epsilon}^{\ell}\right\|^{2} \\
& +\left(2-\delta_{1}\right) v \mathbb{E}\left(k \sum_{\ell=1}^{M}\left\|\nabla \tilde{\boldsymbol{\epsilon}}^{\ell}\right\|^{2}\right)+\frac{1}{\varepsilon} \mathbb{E}\left(k \sum_{\ell=1}^{M}\left\|\operatorname{div} \tilde{\boldsymbol{\epsilon}}^{\ell}\right\|^{2}\right) \\
& \leq C_{\delta_{1}} v \sum_{\ell=1}^{M} \int_{t_{\ell-1}}^{t_{\ell}} \mathbb{E}\left\|\nabla\left(\boldsymbol{z}\left(t_{\ell}\right)-\boldsymbol{z}(s)\right)\right\|^{2}+\varepsilon \sum_{\ell=1}^{M} \int_{t_{\ell-1}}^{t_{\ell}} \mathbb{E}\|\pi(s)\|^{2} d s .
\end{aligned}
$$

Finally, we choose $\delta_{1}>0$ so that $\left(2-\delta_{1}\right)$ stays positive and conclude the proof with Lemma 2.4, Proposition 2.3 and (2.14), and the stability of $\boldsymbol{P}_{\mathbb{H}}$ in $\mathbb{W}^{1,2}$.

Lemma 3.5. Let $\alpha>1$ and $0<\eta<1 / 2$. For every $\varepsilon>0$, there exists a constant $C=C(T, v, \eta)>0$ such that we have

$$
\mathbb{E}\left(k \sum_{\ell=1}^{M}\left\|\omega^{\ell}\right\|^{2}\right) \leq C\left(k^{\eta}+\varepsilon\right) .
$$

Proof. We substitute (3.63) to (3.62) and arrange the result such that we obtain

$$
\begin{aligned}
\left(\boldsymbol{\epsilon}^{\ell}-\boldsymbol{\epsilon}^{\ell-1}, \boldsymbol{\varphi}\right) & +v k\left(\nabla \tilde{\boldsymbol{\epsilon}}^{\ell}, \nabla \boldsymbol{\varphi}\right) \\
& +k\left(\nabla \omega^{\ell}, \boldsymbol{\varphi}\right)=R_{\ell}^{z}(\boldsymbol{\varphi})+\int_{t_{\ell-1}}^{t_{\ell}}\left(\nabla\left(\pi\left(t_{\ell}\right)-\pi(s)\right), \boldsymbol{\varphi}\right) d s .
\end{aligned}
$$


Using identity (3.59), we get

$$
k\left(\nabla \omega^{\ell}, \boldsymbol{\varphi}\right)=\left(\boldsymbol{\epsilon}^{\ell-1}-\boldsymbol{\epsilon}^{\ell}, \boldsymbol{\varphi}\right)+\int_{t_{\ell-1}}^{t_{\ell}}\left(\nabla\left(\pi\left(t_{\ell}\right)-\pi(s)\right), \boldsymbol{\varphi}\right) d s-k v\left(\nabla \tilde{\boldsymbol{\epsilon}}^{\ell}, \nabla \boldsymbol{\varphi}\right)+R_{\ell}^{z}(\boldsymbol{\varphi}) .
$$

Using inequality (3.46), we derive that

$$
\begin{aligned}
k^{2}\left\|\omega^{\varepsilon, \ell}\right\|^{2} \leq C\left\|R_{\ell}^{z}\right\|_{-1}^{2} & +C\left\|\epsilon^{\ell}-\epsilon^{\ell-1}\right\|_{-1}^{2}+(v k)^{2}\left\|\nabla \boldsymbol{\epsilon}^{\ell}\right\|^{2} \\
& +C k \int_{t_{\ell-1}}^{t_{\ell}}\left\|\pi\left(t_{\ell}\right)-\pi(s)\right\|^{2} d s .
\end{aligned}
$$

For brevity let us introduce the numbering

$$
\begin{aligned}
\mathrm{I}+\mathrm{II}+\mathrm{III}+\mathrm{IV} \\
\quad:=C\left\|R_{\ell}^{z}\right\|_{-1}^{2}+C\left\|\boldsymbol{\epsilon}^{\ell}-\epsilon^{\ell-1}\right\|_{-1}^{2}+C k \int_{t_{\ell-1}}^{t_{\ell}}\left\|\pi\left(t_{\ell}\right)-\pi(s)\right\|^{2} d s+(v k)^{2}\left\|\nabla \boldsymbol{\epsilon}^{\ell}\right\|^{2} .
\end{aligned}
$$

First, we have for I

$$
\begin{aligned}
I=\sup _{\boldsymbol{\varphi} \in \mathbb{W}^{1,2}} \frac{\left(R_{\ell}^{z}(\boldsymbol{\varphi})\right)^{2}}{\|\boldsymbol{\varphi}\|_{1}^{2}} & =\left(\int_{t_{\ell-1}}^{t_{\ell}} \sup _{\boldsymbol{\varphi} \in \mathbb{W}^{1,2}} v \frac{\left(\nabla\left(z\left(t_{\ell}\right)-z(s)\right), \nabla \boldsymbol{\varphi}\right)}{\|\boldsymbol{\varphi}\|_{1}} d s\right)^{2} \\
& \leq C(v, L) k \int_{t_{\ell-1}}^{t_{\ell}} \| \nabla\left(z\left(t_{\ell}\right)-z(s) \|^{2} d s .\right.
\end{aligned}
$$

Now, we estimate the term II. Since $\epsilon^{\ell}-\epsilon^{\ell-1} \in \mathcal{D}\left(A^{-1}\right)$, we can take $\varphi=A^{-1}\left(\boldsymbol{\epsilon}^{\ell}-\boldsymbol{\epsilon}^{\ell-1}\right)$ in identity (3.74). From the orthogonality we get

$$
\begin{aligned}
& k\left(\nabla \omega^{\ell}, A^{-1}\left(\boldsymbol{\epsilon}^{\ell}-\boldsymbol{\epsilon}^{\ell-1}\right)\right)=\int_{t_{\ell-1}}^{t_{\ell}}\left(\nabla\left(\pi\left(t_{\ell}\right)-\pi(s)\right), A^{-1}\left(\boldsymbol{\epsilon}^{\ell}-\boldsymbol{\epsilon}^{\ell-1}\right)\right) d s=0, \\
& k\left(\nabla \omega^{\ell}, A^{-1}\left(\boldsymbol{\epsilon}^{\ell}-\boldsymbol{\epsilon}^{\ell-1}\right)\right)=\int_{t_{\ell-1}}^{t_{\ell}}\left(\nabla\left(\pi\left(t_{\ell}\right)-\pi(s)\right), A^{-1}\left(\boldsymbol{\epsilon}^{\ell}-\boldsymbol{\epsilon}^{\ell-1}\right)\right) d s=0,
\end{aligned}
$$

and from Proposition 2.1 we get

$$
\text { II } \leq C\left(\epsilon^{\ell}-\epsilon^{\ell-1}, A^{-1}\left(\epsilon^{\ell}-\epsilon^{\ell-1}\right)\right) .
$$

Applying the Young inequality we obtain the following results:

$$
R_{\ell}^{z}\left(A^{-1}\left(\boldsymbol{\epsilon}^{\ell}-\boldsymbol{\epsilon}^{\ell-1}\right)\right) \leq C\left(\delta_{1}, v\right) k \int_{t_{\ell-1}}^{t_{\ell}}\left\|\nabla\left(z\left(t_{\ell}\right)-z(s)\right)\right\|^{2} d s+\delta_{1} \mathrm{II} .
$$

Collecting the last four estimates we obtain

$$
\left(1-2 \delta_{1}\right) \mathrm{II} \leq C\left(\delta_{1}\right) k^{2}\left\|\nabla \tilde{\boldsymbol{\epsilon}}^{\ell}\right\|^{2}+C\left(\delta_{1}, v\right) k \int_{t_{\ell-1}}^{t_{\ell}}\left\|\nabla\left(z\left(t_{\ell}\right)-z(s)\right)\right\|^{2} d s .
$$

After choosing $\delta_{1}$ so that $\left(1-2 \delta_{1}\right)>0$, we substitute the estimates of I and II in (3.75), let the terms $I I$ and $I V$ unchanged, and get in this way the following new estimate

$$
\begin{aligned}
k^{2}\left\|\omega^{\ell}\right\|^{2} & \leq C(v, L) k \int_{t_{\ell-1}}^{t_{\ell}} \| \nabla\left(z\left(t_{\ell}\right)-z(s)\left\|^{2} d s+C(v) k^{2}\right\| \nabla \tilde{\boldsymbol{e}}^{\ell} \|^{2}\right. \\
& +C k \int_{t_{\ell-1}}^{t_{\ell}}\left\|\nabla\left(z\left(t_{\ell}\right)-z(s)\right)\right\|^{2} d s+C k \int_{t_{\ell-1}}^{t_{\ell}}\left\|\pi\left(t_{\ell}\right)-\pi(s)\right\|^{2} d s .
\end{aligned}
$$

By taking the sum for $\ell=1$ to $\ell=M$ and expectation in (3.82), we get

$$
\begin{aligned}
& k \mathbb{E}\left(k \sum_{\ell=1}^{M}\left\|\omega^{\ell}\right\|^{2}\right) \leq C(v, L) k \sum_{\ell=1}^{M} \int_{t_{\ell-1}}^{t_{\ell}} \mathbb{E} \| \nabla\left(z\left(t_{\ell}\right)-z(s) \|^{2} d s++C(v) k \mathbb{E}\left(k \sum_{\ell=1}^{M}\left\|\nabla \tilde{\boldsymbol{\epsilon}}^{\ell}\right\|^{2}\right)\right. \\
& \quad+C(v) k \sum_{\ell=1}^{M} \int_{t_{\ell-1}}^{t_{\ell}} \mathbb{E}\left\|\nabla\left(z\left(t_{\ell}\right)-\boldsymbol{z}(s)\right)\right\|^{2} d s+C k \sum_{\ell=1}^{M} \int_{t_{\ell-1}}^{t_{\ell}} \mathbb{E}\left\|\pi\left(t_{\ell}\right)-\pi(s)\right\|^{2} d s .
\end{aligned}
$$


From Lemma 2.4 (iii) and Lemma 3.4 we obtain

$$
k \mathbb{E}\left(k \sum_{\ell=1}^{M}\left\|\omega^{\ell}\right\|^{2}\right) \leq C(L, T, v)\left(k^{\eta+1}+k\left(k^{\eta}+\varepsilon\right)\right) .
$$

Let $v=\left\{v(t, \cdot): t \in\left[t_{\ell-1}, t_{\ell}\right]\right\}$ be the strong solution of (2.21) as defined in Definition 2.2 and $\rho=$ $\left\{\rho(t, \cdot): t \in\left[t_{\ell-1}, t_{\ell}\right]\right\}$ the associated pressure, i.e. for every $t \in\left[t_{\ell-1}, t_{\ell}\right]$ and all $\varphi \in \mathbb{W}^{1,2}, \chi \in L_{\text {per }}^{2}$, we have $\mathbb{P}$-a.s.

$$
\begin{aligned}
\left(\boldsymbol{v}\left(t_{\ell}\right), \boldsymbol{\varphi}\right)+v \int_{t_{\ell-1}}^{t_{\ell}}(\nabla \boldsymbol{v}(s), \nabla \boldsymbol{\varphi}) d s+\int_{t_{\ell-1}}^{t_{\ell}} \tilde{b}(\boldsymbol{u}(s), \boldsymbol{u}(s), \boldsymbol{\varphi}) d s & \\
+\int_{t_{\ell-1}}^{t_{\ell}}(\nabla \rho(s), \boldsymbol{\varphi}) d s & =\int_{t_{\ell-1}}^{t_{\ell}}(\boldsymbol{\varphi}, d \boldsymbol{W}(s)), \\
\left(\operatorname{div} \boldsymbol{v}\left(t_{\ell}\right), \chi\right) & =0 .
\end{aligned}
$$

To these equations correspond the following algorithm:

Algorithm 4 (Second auxiliary algorithm). Let $\boldsymbol{v}^{0}:=\boldsymbol{u}_{0}$ be a given $\mathbb{V}$-valued random variable. Find for every $\ell \in\{1, \ldots, M\}$ a tuple of random variables $\left(\boldsymbol{v}^{\varepsilon, \ell}, \rho^{\varepsilon, \ell}\right)$ with values in $\mathbb{W}_{\text {per }}^{1,2} \times L_{\text {per }}^{2}$, such that we have $\mathbb{P}$-a.s.

- Penalization:

$$
\begin{aligned}
\left(\tilde{\boldsymbol{v}}^{\varepsilon, \ell}-\boldsymbol{v}^{\varepsilon, \ell-1}, \boldsymbol{\varphi}\right)+v k\left(\nabla \tilde{\boldsymbol{v}}^{\varepsilon, \ell}, \nabla \boldsymbol{\varphi}\right)+k \tilde{b}\left(\tilde{\boldsymbol{v}}^{\varepsilon, \ell}+\tilde{\boldsymbol{z}}^{\varepsilon, \ell}, \tilde{\boldsymbol{v}}^{\varepsilon, \ell}+\tilde{\boldsymbol{z}}^{\varepsilon, \ell}, \boldsymbol{\varphi}\right) & \\
+k\left(\nabla \tilde{\rho}^{\varepsilon, \ell}, \boldsymbol{\varphi}\right)+k\left(\nabla \psi^{\varepsilon, \ell-1}, \boldsymbol{\varphi}\right) & =0, \forall \boldsymbol{\varphi} \in \mathbb{W}^{1,2}, \\
\left(\operatorname{div} \tilde{\boldsymbol{v}}^{\varepsilon, \ell}, \chi\right)+\varepsilon\left(\tilde{\rho}^{\varepsilon, \ell}, \chi\right) & =0, \forall \chi \in L_{\mathrm{per}}^{2}
\end{aligned}
$$

- Projection:

$$
\begin{aligned}
\left(\boldsymbol{v}^{\varepsilon, \ell}-\tilde{\boldsymbol{v}}^{\varepsilon, \ell}, \boldsymbol{\varphi}\right)+\alpha k\left(\nabla\left(\psi^{\varepsilon, \ell}-\psi^{\varepsilon, \ell-1}\right), \boldsymbol{\varphi}\right) & =0, \forall \boldsymbol{\varphi} \in \mathbb{W}_{\text {per }}^{1,2} \\
\left(\operatorname{div} \boldsymbol{v}^{\varepsilon, \ell}, \chi\right) & =0, \forall \chi \in L_{\text {per }}^{2} \\
\rho^{\varepsilon, \ell} & =\tilde{\rho}^{\varepsilon, \ell}+\psi^{\varepsilon, \ell}+\alpha\left(\psi^{\varepsilon, \ell}-\psi^{\varepsilon, \ell-1}\right) .
\end{aligned}
$$

Define the errors $\sigma^{\ell}=v\left(t_{\ell}\right)-v^{\varepsilon, \ell}, \tilde{\sigma}^{\ell}=v\left(t_{\ell}\right)-\tilde{v}^{\varepsilon, \ell}, \tilde{\varrho}^{\ell}=\rho\left(t_{\ell}\right)-\tilde{\rho}^{\varepsilon, \ell}$, and $\varrho^{\ell}=\rho\left(t_{\ell}\right)-\rho^{\varepsilon, \ell}$. Subtracting (3.85) from (3.83) we get

$$
\begin{gathered}
\left(\tilde{\boldsymbol{\sigma}}^{\ell}-\boldsymbol{\sigma}^{\ell-1}, \boldsymbol{\varphi}\right)+v \int_{t_{\ell-1}}^{t_{\ell}}\left(\nabla\left(\boldsymbol{v}(s)-\tilde{\boldsymbol{v}}^{\varepsilon, \ell}\right), \nabla \boldsymbol{\varphi}\right) d s+\int_{t_{\ell-1}}^{t_{\ell}} \tilde{b}(\boldsymbol{u}(s), \boldsymbol{u}(s), \boldsymbol{\varphi}) d s \\
-\int_{t_{\ell-1}}^{t_{\ell}} \tilde{b}\left(\boldsymbol{u}^{\ell}, \boldsymbol{u}^{\ell}, \boldsymbol{\varphi}\right) d s+\int_{t_{\ell-1}}^{t_{\ell}}\left(\nabla\left(\rho(s)-\tilde{\rho}^{\varepsilon, \ell}\right), \boldsymbol{\varphi}\right) d s-k\left(\nabla \psi^{\varepsilon, \ell-1}, \boldsymbol{\varphi}\right)=0 .
\end{gathered}
$$

Choosing $\chi=\operatorname{div} \tilde{v}^{\varepsilon, \ell}$ in (3.86) we get

$$
-\left(\nabla \tilde{\rho}^{\varepsilon, \ell}, \boldsymbol{\varphi}\right)=\frac{1}{\varepsilon}\left(\nabla \operatorname{div} \tilde{v}^{\varepsilon, \ell}, \boldsymbol{\varphi}\right) .
$$

Thanks to the identities

$$
\begin{aligned}
\left(\nabla\left(\boldsymbol{v}(s)-\tilde{\boldsymbol{v}}^{\varepsilon, \ell}\right), \nabla \boldsymbol{\varphi}\right) & =\left(\nabla \tilde{\boldsymbol{\sigma}}^{\ell}, \nabla \boldsymbol{\varphi}\right)+\left(\nabla\left(\boldsymbol{v}(s)-\boldsymbol{v}\left(t_{\ell}\right)\right), \nabla \boldsymbol{\varphi}\right) \text { and } \\
\left(\nabla\left(\rho(s)-\tilde{\rho}^{\varepsilon, \ell}\right), \boldsymbol{\varphi}\right) & =\left(\nabla(\rho(s), \boldsymbol{\varphi})+\frac{1}{\varepsilon}\left(\nabla \operatorname{div} \tilde{\boldsymbol{v}}^{\varepsilon, \ell}, \boldsymbol{\varphi}\right),\right.
\end{aligned}
$$

equation (3.89) is reduced to

$$
\begin{aligned}
\left(\tilde{\boldsymbol{\sigma}}^{\ell}-\sigma^{\ell-1}, \boldsymbol{\varphi}\right) & +v k\left(\nabla \tilde{\boldsymbol{\sigma}}^{\ell}, \nabla \boldsymbol{\varphi}\right)+\frac{k}{\varepsilon}\left(\nabla \operatorname{div} \tilde{\boldsymbol{v}}^{\varepsilon, \ell}, \boldsymbol{\varphi}\right) \\
& -k\left(\nabla \psi^{\varepsilon, \ell-1}, \boldsymbol{\varphi}\right)=Q_{\ell}(\boldsymbol{\varphi})+R_{\ell}^{v}(\boldsymbol{\varphi})+\int_{t_{\ell-1}}^{t_{\ell}}\left(\operatorname{div} \tilde{\boldsymbol{\sigma}}^{\ell}, \rho(s)\right) d s,
\end{aligned}
$$


where

$$
\begin{aligned}
& Q_{\ell}(\boldsymbol{\varphi})=\int_{t_{\ell-1}}^{t_{\ell}}\left(\tilde{b}(\boldsymbol{u}(s), \boldsymbol{u}(s), \boldsymbol{\varphi})-\tilde{b}\left(\tilde{\boldsymbol{u}}^{\varepsilon, \ell}, \tilde{\boldsymbol{u}}^{\varepsilon, \ell}, \boldsymbol{\varphi}\right)\right) d s, \\
& R_{\ell}^{v}(\boldsymbol{\varphi})=v \int_{t_{\ell-1}}^{t_{\ell}}\left(\nabla\left(\boldsymbol{v}\left(t_{\ell}\right)-\boldsymbol{v}(s)\right), \nabla \boldsymbol{\varphi}\right) d s .
\end{aligned}
$$

To (3.93) we associate the following projection equation

$$
\left\{\begin{aligned}
\left(\sigma^{\ell}-\tilde{\sigma}^{\ell}, \boldsymbol{\varphi}\right) & =k \alpha\left(\nabla\left(\psi^{\varepsilon, \ell}-\psi^{\varepsilon, \ell-1}\right), \boldsymbol{\varphi}\right), \\
\operatorname{div} \sigma^{\ell} & =0 .
\end{aligned}\right.
$$

Let $\kappa_{1}, \kappa_{2}, \kappa_{3}>0$ some fixed constants, and let us introduce the sample subsets

$$
\begin{aligned}
& \Omega_{\kappa_{1}}=\left\{\omega \in \Omega: \sup _{0 \leq t \leq T}\|\boldsymbol{u}(t)\|_{\mathbb{V}}^{2}+k \sum_{\ell=1}^{M}\left\|\boldsymbol{u}^{\ell}\right\|_{1}^{2} \leq \kappa_{1}\right\}, \\
& \Omega_{\kappa_{2}}=\left\{\omega \in \Omega: \max _{1 \leq m \leq M}\left\|\boldsymbol{\epsilon}^{m}\right\|^{2}+v k \sum_{\ell=1}^{M}\left\|\boldsymbol{\epsilon}^{\ell}\right\|_{1}^{2}+k \sum_{\ell=1}^{M}\left\|\omega^{\ell}\right\|^{2} \leq \kappa_{2}\right\}, \text { and } \\
& \Omega_{\kappa_{3}}=\left\{\omega \in \Omega: \forall 0 \leq s<t \leq T,\|\boldsymbol{u}(s)-\boldsymbol{u}(t)\|_{\mathbb{L}^{4}}^{2} \leq \kappa_{3}|t-s|^{2 \eta}\right\} .
\end{aligned}
$$

In the next paragraph we derive some error estimates on the intersection of these subsets of $\Omega$.

Lemma 3.6. Let $\alpha>1$ and $0<\eta<1 / 2$. For every $\varepsilon>0$, there exists a constant $C=C(L, T, v)>0$ such that on $\Omega_{\kappa_{1}} \cap \Omega_{\kappa_{2}} \cap \Omega_{\kappa_{3}}$ we have

$$
\max _{1<m \leq M}\left\|\sigma^{m}\right\|^{2}+v k \sum_{\ell=1}^{M}\left\|\nabla \sigma^{\ell}\right\|^{2} \leq C\left(\kappa_{1} \kappa_{3} k^{2 \eta}+\kappa_{1} \kappa_{2}+\kappa_{2}^{2}+k^{\eta}+\varepsilon\right) \exp \left(\kappa_{1}\right) .
$$

Proof. We take $\varphi=2 \tilde{\sigma}^{\ell}$ in (3.93) and proceed exactly like in the proof of Lemma 3.4 until (3.72). Doing so we arrive at

$$
\begin{array}{r}
\max _{1 \leq m \leq M}\left\{\left(\frac{\alpha+1}{2 \alpha}\right)\left\|\sigma^{m}\right\|^{2}+\left(\frac{\alpha-1}{2 \alpha}\right)\left\|\tilde{\sigma}^{m}\right\|^{2}\right\}+\left(\frac{\alpha-1}{2 \alpha}\right) \sum_{\ell=1}^{M-1}\left\|\tilde{\sigma}^{\ell}-\sigma^{\ell}\right\|^{2} \\
+\left(v-\delta_{1}\right) k \sum_{\ell=1}^{M}\left\|\nabla \tilde{\sigma}^{\ell}\right\|^{2} \leq C\left(\delta_{1}\right) v \sum_{\ell=1}^{M} \int_{t_{\ell-1}}^{t_{\ell}}\left\|\nabla\left(v\left(t_{\ell}\right)-v(s)\right)\right\|^{2} \\
+\varepsilon \sum_{\ell=1}^{M} \int_{t_{\ell-1}}^{t_{\ell}}\|\rho(s)\|^{2} d s+2 \max _{1 \leq m \leq M} \sum_{\ell=1}^{m} Q_{\ell}\left(\tilde{\sigma}^{\ell}\right),
\end{array}
$$

where

$$
Q_{\ell}\left(\tilde{\sigma}^{\ell}\right)=\int_{t_{\ell-1}}^{t_{\ell}} \tilde{b}\left(\boldsymbol{u}(s), \boldsymbol{u}(s), \tilde{\sigma}^{\ell}\right)-\tilde{b}\left(\tilde{\boldsymbol{u}}^{\varepsilon, \ell}, \tilde{\boldsymbol{u}}^{\varepsilon, \ell}, \tilde{\sigma}^{\ell}\right) d s .
$$

We split the term $Q_{\ell}$ into four terms as follows

$$
\begin{aligned}
Q_{\ell}\left(\tilde{\boldsymbol{\sigma}}^{\ell}\right) \leq \int_{t_{\ell-1}}^{t_{\ell}}\left(\tilde{b}\left(\boldsymbol{u}(s), \boldsymbol{u}(s)-\boldsymbol{u}\left(t_{\ell}\right), \tilde{\boldsymbol{\sigma}}^{\ell}\right)+\tilde{b}\left(\boldsymbol{u}(s)-\boldsymbol{u}\left(t_{\ell}\right), \boldsymbol{u}\left(t_{\ell}\right), \tilde{\boldsymbol{\sigma}}^{\ell}\right)\right. \\
\left.\quad+\tilde{b}\left(\boldsymbol{u}\left(t_{\ell}\right), \boldsymbol{u}\left(t_{\ell}\right)-\tilde{\boldsymbol{u}}^{\varepsilon, \ell}, \tilde{\boldsymbol{\sigma}}^{\ell}\right)+\tilde{b}\left(\boldsymbol{u}\left(t_{\ell}\right)-\tilde{\boldsymbol{u}}^{\varepsilon, \ell}, \tilde{\boldsymbol{u}}^{\varepsilon, \ell}, \tilde{\boldsymbol{\sigma}}^{\ell}\right)\right) d s \\
\leq \int_{t_{\ell-1}}^{t_{\ell}}\left(\operatorname{NLT}_{1}\left(\tilde{\boldsymbol{\sigma}}^{\ell}\right)+N L T_{2}\left(\tilde{\boldsymbol{\sigma}}^{\ell}\right)+\operatorname{NLT}_{3}\left(\tilde{\boldsymbol{\sigma}}^{\ell}\right)+N L T_{4}\left(\tilde{\boldsymbol{\sigma}}^{\ell}\right)\right) d s .
\end{aligned}
$$

In the next lines, we will estimate the terms $N L T_{j}\left(\tilde{\sigma}^{\ell}\right), j=1, \ldots, 4$, one by one.

- $\operatorname{NLT}_{1}\left(\tilde{\sigma}^{\ell}\right)$ : From (2.9), the Sobolev embedding $\mathbb{W}^{1,2}(D) \subset \mathbb{L}^{4}(D)$, and the Young inequality, we get the estimate

$$
N L T_{1}\left(\tilde{\boldsymbol{\sigma}}^{\ell}\right) \leq\left|\tilde{b}\left(\boldsymbol{u}(s), \tilde{\boldsymbol{\sigma}}^{\ell}, \boldsymbol{u}(s)-\boldsymbol{u}\left(t_{\ell}\right)\right)\right| \leq C\left(\delta_{1}, L\right)\|\boldsymbol{u}(s)\|_{1}^{2}\left\|\boldsymbol{u}(s)-\boldsymbol{u}\left(t_{\ell}\right)\right\|_{\mathbb{L}^{4}}^{2}+\delta_{1}\left\|\tilde{\boldsymbol{\sigma}}^{\ell}\right\|_{1}^{2} .
$$


Then, integrating over the time interval $\left[t_{\ell-1}, t_{\ell}\right]$ with respect to $s$, using the Hölder inequality, and since $\omega \in \Omega_{\kappa_{1}} \cap \Omega_{\kappa_{2}} \cap \Omega_{\kappa_{3}}$, we get

$$
\begin{aligned}
\int_{t_{\ell-1}}^{t_{\ell}} N L T_{1}\left(\tilde{\boldsymbol{\sigma}}^{\ell}\right) d s & \leq C\left(\delta_{1}, L\right) \int_{t_{\ell-1}}^{t_{\ell}}\|\boldsymbol{u}(s)\|_{1}^{2}\left\|\boldsymbol{u}(s)-\boldsymbol{u}\left(t_{\ell}\right)\right\|_{\mathbb{L}^{4}}^{2} d s+\delta_{1} k\left\|\tilde{\boldsymbol{\sigma}}^{\ell}\right\|_{1}^{2} \\
& \leq C\left(\delta_{1}, L\right) \sup _{t_{\ell-1} \leq s \leq t_{\ell}}\|\boldsymbol{u}(s)\|_{1}^{2} \int_{t_{\ell-1}}^{t_{\ell}}\left\|\boldsymbol{u}(s)-\boldsymbol{u}\left(t_{\ell}\right)\right\|_{\mathbb{L}^{4}}^{2} d s+\delta_{1} k\left\|\tilde{\boldsymbol{\sigma}}^{\ell}\right\|_{1}^{2} \\
& \leq C\left(\delta_{1}, L\right) \sup _{t_{\ell-1} \leq s \leq t_{\ell}}\|\boldsymbol{u}(s)\|_{1}^{2} \int_{t_{\ell-1}}^{t_{\ell}} \kappa_{3}\left|s-t_{\ell}\right|^{2 \eta} d s+\delta_{1} k\left\|\tilde{\boldsymbol{\sigma}}^{\ell}\right\|_{1}^{2} \\
& \leq C\left(\delta_{1}, L\right) \kappa_{1} \kappa_{3} k^{2 \eta+1}+\delta_{1} k\left\|\tilde{\boldsymbol{\sigma}}^{\ell}\right\|_{1}^{2} .
\end{aligned}
$$

- $\operatorname{NLT}_{2}\left(\tilde{\sigma}^{\ell}\right)$ : Again from (2.9) and the Young inequality, we infer

$$
N L T_{2}\left(\tilde{\boldsymbol{\sigma}}^{\ell}\right) \leq\left|\tilde{b}\left(\boldsymbol{u}(s)-\boldsymbol{u}\left(t_{\ell}\right), \boldsymbol{u}\left(t_{\ell}\right), \tilde{\boldsymbol{\sigma}}^{\ell}\right)\right| \leq C\left(\delta_{1}\right)\left\|\boldsymbol{u}(s)-\boldsymbol{u}\left(t_{\ell}\right)\right\|_{\mathbb{L}^{4}}^{2}\left\|\boldsymbol{u}\left(t_{\ell}\right)\right\|_{1}^{2}+\delta_{1}\left\|\tilde{\boldsymbol{\sigma}}^{\ell}\right\|_{1}^{2} .
$$

Again, integrating over the time interval $\left[t_{\ell-1}, t_{\ell}\right]$ with respect to $s$ and since $\omega \in \Omega_{\kappa_{2}}$, we get

$$
\begin{aligned}
\int_{t_{\ell-1}}^{t_{\ell}} N L T_{2}\left(\tilde{\sigma}^{\ell}\right) d s & \leq C\left(\delta_{1}\right)\left\|\boldsymbol{u}\left(t_{\ell}\right)\right\|_{1}^{2} \int_{t_{\ell-1}}^{t_{\ell}}\left\|\boldsymbol{u}(s)-\boldsymbol{u}\left(t_{\ell}\right)\right\|_{\mathbb{L}^{4}}^{2} d s+\delta_{1} k\left\|\tilde{\sigma}^{\ell}\right\|_{1}^{2} \\
& \leq C\left(\delta_{1}\right)\left\|\boldsymbol{u}\left(t_{\ell}\right)\right\|_{1}^{2} \int_{t_{\ell-1}}^{t_{\ell}} \kappa_{3}\left|s-t_{\ell}\right|^{2 \eta} d s+\delta_{1} k\left\|\tilde{\boldsymbol{\sigma}}^{\ell}\right\|_{1}^{2} \\
& \leq C\left(\delta_{1}\right) \kappa_{3} k^{2 \eta+1}\left\|\boldsymbol{u}\left(t_{\ell}\right)\right\|_{1}^{2}+\delta_{1} k\left\|\tilde{\boldsymbol{\sigma}}^{\ell}\right\|_{1}^{2} .
\end{aligned}
$$

Summing up from $\ell=1$ to $\ell=M$, using the Hölder inequality, and since $\omega \in \Omega_{\kappa_{1}}$, we get

$$
\begin{aligned}
\sum_{\ell=1}^{M} \int_{t_{\ell-1}}^{t_{\ell}} N L T_{2}\left(\tilde{\boldsymbol{\sigma}}^{\ell}\right) d s & \leq C\left(\delta_{1}\right) \kappa_{3} k^{2 \eta+1} \sum_{\ell=1}^{M}\left\|\boldsymbol{u}\left(t_{\ell}\right)\right\|_{1}^{2}+\delta_{1} k \sum_{\ell=1}^{M}\left\|\boldsymbol{\sigma}^{\ell}\right\|_{1}^{2} \\
& \leq C\left(\delta_{1}, T\right) \kappa_{1} \kappa_{3} k^{2 \eta}+\delta_{1} k \sum_{\ell=1}^{M}\left\|\boldsymbol{\sigma}^{\ell}\right\|_{1}^{2} .
\end{aligned}
$$

- $\operatorname{NLT}_{3}\left(\tilde{\boldsymbol{\sigma}}^{\ell}\right)$ : Since $\boldsymbol{u}\left(t_{\ell}\right)-\tilde{\boldsymbol{u}}^{\varepsilon, \ell}=\tilde{\boldsymbol{\epsilon}}^{\ell}+\tilde{\boldsymbol{\sigma}}^{\ell}$ and thanks to the orthogonal property of $\tilde{b}$ (see Eq. (2.8)), we have

$$
N L T_{3}\left(\tilde{\sigma}^{\ell}\right)=\left|\tilde{b}\left(\boldsymbol{u}\left(t_{\ell}\right), \boldsymbol{u}\left(t_{\ell}\right)-\tilde{\boldsymbol{u}}^{\varepsilon, \ell}, \tilde{\boldsymbol{\sigma}}^{\ell}\right)\right|=\left|\tilde{b}\left(\boldsymbol{u}\left(t_{\ell}\right), \tilde{\boldsymbol{\epsilon}}^{\ell}+\tilde{\boldsymbol{\sigma}}^{\ell}, \tilde{\boldsymbol{\sigma}}^{\ell}\right)\right|=\left|\tilde{b}\left(\boldsymbol{u}\left(t_{\ell}\right), \tilde{\boldsymbol{\epsilon}}^{\ell}, \tilde{\boldsymbol{\sigma}}^{\ell}\right)\right| .
$$

From (2.10) and the Young inequality, we have

$$
\operatorname{NLT}_{3}\left(\tilde{\boldsymbol{\sigma}}^{\ell}\right) \leq C\left(\delta_{1}, L\right)\left\|\boldsymbol{u}\left(t_{\ell}\right)\right\|_{1}^{2}\left\|\tilde{\boldsymbol{\epsilon}}^{\ell}\right\|_{1}^{2}+\delta_{1}\left\|\tilde{\boldsymbol{\sigma}}^{\ell}\right\|_{1}^{2} .
$$

As before, integrating over the interval $\left[t_{\ell-1}, t_{\ell}\right]$ with respect to $s$, we obtain

$$
\int_{t_{\ell-1}}^{t_{\ell}} N L T_{3}\left(\tilde{\boldsymbol{\sigma}}^{\ell}\right) d s \leq C_{\delta_{1}}(L) k\left\|\boldsymbol{u}\left(t_{\ell}\right)\right\|_{1}^{2}\left\|\tilde{\boldsymbol{\epsilon}}^{\ell}\right\|_{1}^{2}+\delta_{1} k\left\|\tilde{\boldsymbol{\sigma}}^{\ell}\right\|_{1}^{2} .
$$

Summing up from $\ell=1$ to $\ell=M$, using the Hölder inequality, and since $\omega \in \Omega_{\kappa_{1}} \cap \Omega_{\kappa_{2}}$, we have

$$
\begin{aligned}
\sum_{\ell=1}^{M} \int_{t_{\ell-1}}^{t_{\ell}} N L T_{3}\left(\boldsymbol{\sigma}^{\ell}\right) d s & \leq C\left(\delta_{1}, L\right) k \sum_{\ell=1}^{M}\left\|\boldsymbol{u}\left(t_{\ell}\right)\right\|_{1}^{2}\left\|\tilde{\boldsymbol{\epsilon}}^{\ell}\right\|_{1}^{2}+\delta_{1} k \sum_{\ell=1}^{M}\left\|\tilde{\boldsymbol{\sigma}}^{\ell}\right\|_{1}^{2} \\
& \leq C\left(\delta_{1}, L\right) \max _{1 \leq \ell \leq M}\left\|\boldsymbol{u}\left(t_{\ell}\right)\right\|_{1}^{2}\left(k \sum_{\ell=1}^{M}\left\|\tilde{\boldsymbol{\epsilon}}^{\ell}\right\|_{1}^{2}\right)+\delta_{1} k \sum_{\ell=1}^{M}\left\|\tilde{\boldsymbol{\sigma}}^{\ell}\right\|_{1}^{2} \\
& \leq C\left(\delta_{1}, L\right) \kappa_{1} \kappa_{2}+\delta_{1} k \sum_{\ell=1}^{M}\left\|\tilde{\boldsymbol{\sigma}}^{\ell}\right\|_{1}^{2} .
\end{aligned}
$$

- $N L T_{4}\left(\tilde{\boldsymbol{\sigma}}^{\ell}\right)$ : By similar computations as before and using the fact that $\boldsymbol{u}\left(t_{\ell}\right)-\tilde{\boldsymbol{u}}^{\varepsilon, \ell}=\tilde{\boldsymbol{\epsilon}}^{\ell}+\tilde{\boldsymbol{\sigma}}^{\ell}$, we get

$$
\operatorname{NLT}_{4}\left(\tilde{\boldsymbol{\sigma}}^{\ell}\right)=\left|\tilde{b}\left(\tilde{\boldsymbol{\epsilon}}^{\ell}+\tilde{\boldsymbol{\sigma}}^{\ell}, \tilde{\boldsymbol{u}}^{\varepsilon, \ell}, \tilde{\boldsymbol{\sigma}}^{\ell}\right)\right| \leq\left|\tilde{b}\left(\tilde{\boldsymbol{\epsilon}}^{\ell}, \tilde{\boldsymbol{u}}^{\varepsilon, \ell}, \tilde{\boldsymbol{\sigma}}^{\ell}\right)\right|+\left|\tilde{b}\left(\tilde{\boldsymbol{\sigma}}^{\ell}, \tilde{\boldsymbol{u}}^{\varepsilon, \ell}, \tilde{\boldsymbol{\sigma}}^{\ell}\right)\right|
$$


For simplicity, let us introduce the notation

$$
N L T_{4, a}\left(\tilde{\boldsymbol{\sigma}}^{\ell}\right):=\left|\tilde{b}\left(\tilde{\boldsymbol{\epsilon}}^{\ell}, \tilde{\boldsymbol{u}}^{\varepsilon, \ell}, \tilde{\boldsymbol{\sigma}}^{\ell}\right)\right|
$$

and

$$
\operatorname{NLT}_{4, b}\left(\tilde{\sigma}^{\ell}\right):=\left|\tilde{b}\left(\tilde{\sigma}^{\ell}, \tilde{u}^{\varepsilon, \ell}, \tilde{\sigma}^{\ell}\right)\right|
$$

We split $N L T_{4, a}$ into two terms by replacing $\tilde{\boldsymbol{u}}^{\varepsilon, \ell}$ by $\widetilde{\boldsymbol{u}}\left(t_{\ell}\right)+\tilde{\boldsymbol{\epsilon}}^{\ell}$. Next, we apply (2.10) and (2.11) respectively. Finally, we use the Young inequality to get

$$
\begin{aligned}
N L T_{4, a}\left(\tilde{\boldsymbol{\sigma}}^{\ell}\right) & \leq\left|\tilde{b}\left(\tilde{\boldsymbol{\epsilon}}^{\ell}, \boldsymbol{u}\left(t_{\ell}\right), \tilde{\boldsymbol{\sigma}}^{\ell}\right)\right|+\left|\tilde{b}\left(\tilde{\boldsymbol{\epsilon}}^{\ell}, \tilde{\boldsymbol{\epsilon}}^{\ell}, \tilde{\boldsymbol{\sigma}}^{\ell}\right)\right| \\
& \leq C\left(\delta_{1}, L\right)\left\|\tilde{\boldsymbol{\epsilon}}^{\ell}\right\|_{1}^{2}\left\|\boldsymbol{u}\left(t_{\ell}\right)\right\|_{1}^{2}+C\left(\delta_{1}, L\right)\left\|\tilde{\boldsymbol{\epsilon}}^{\ell}\right\|^{2}\left\|\tilde{\boldsymbol{\epsilon}}^{\ell}\right\|_{1}^{2}+\delta_{1}\left\|\tilde{\boldsymbol{\sigma}}^{\ell}\right\|_{1}^{2} .
\end{aligned}
$$

The term $N L T_{4, b}\left(\tilde{\sigma}^{\ell}\right)$ satisfies the skew-symmetry property (see (2.7)). Therefore, using the estimate (2.11) and the Young inequality, we get

$$
\begin{aligned}
\operatorname{NLT}_{4, b}\left(\tilde{\boldsymbol{\sigma}}^{\ell}\right)=\left|\tilde{b}\left(\tilde{\boldsymbol{\sigma}}^{\ell}, \tilde{\boldsymbol{\sigma}}^{\ell}, \tilde{\boldsymbol{u}}^{\varepsilon, \ell}\right)\right| & \leq C(L)\left\|\tilde{\boldsymbol{\sigma}}^{\ell}\right\|\left\|\tilde{\boldsymbol{\sigma}}^{\ell}\right\|_{1}\left\|\tilde{\boldsymbol{u}}^{\varepsilon, \ell}\right\|_{1} \\
& \leq C\left(\delta_{1}, L\right)\left\|\tilde{\boldsymbol{\sigma}}^{\ell}\right\|^{2}\left\|\tilde{\boldsymbol{u}}^{\varepsilon, \ell}\right\|_{1}^{2}+\delta_{1}\left\|\tilde{\boldsymbol{\sigma}}^{\ell}\right\|_{1}^{2} .
\end{aligned}
$$

From these estimates, we obtain after an integration over the time interval $\left[t_{\ell-1}, t_{\ell}\right]$ with respect to $s$ the estimate

$$
\begin{array}{r}
\int_{t_{\ell-1}}^{t_{\ell}} \operatorname{NLT}_{4}\left(\tilde{\boldsymbol{\sigma}}^{\ell}\right) d s \leq C\left(\delta_{1}, L\right) k\left\|\tilde{\boldsymbol{\epsilon}}^{\ell}\right\|_{1}^{2}\left\|\boldsymbol{u}\left(t_{\ell}\right)\right\|_{1}^{2}+C\left(\delta_{1}, L\right) k\left\|\tilde{\boldsymbol{\epsilon}}^{\ell}\right\|^{2}\left\|\tilde{\boldsymbol{\epsilon}}^{\ell}\right\|_{1}^{2} \\
+C\left(\delta_{1}, L\right) k\left\|\boldsymbol{\sigma}^{\ell}\right\|^{2}\left\|\boldsymbol{u}^{\ell}\right\|_{1}^{2}+\delta_{1} k\left\|\boldsymbol{\sigma}^{\ell}\right\|_{1}^{2} .
\end{array}
$$

Then, summing up,

$$
\begin{aligned}
\sum_{\ell=1}^{M} \int_{t_{\ell-1}}^{t_{\ell}} N L T_{4}\left(\tilde{\boldsymbol{\sigma}}^{\ell}\right) d s & \leq C\left(\delta_{1}, L\right) k \sum_{\ell=1}^{M}\left[\left\|\tilde{\boldsymbol{\epsilon}}^{\ell}\right\|_{1}^{2}\left\|\boldsymbol{u}\left(t_{\ell}\right)\right\|_{1}^{2}+\left\|\tilde{\boldsymbol{\epsilon}}^{\ell}\right\|^{2}\left\|\tilde{\boldsymbol{\epsilon}}^{\ell}\right\|_{1}^{2}+\left\|\tilde{\boldsymbol{\sigma}}^{\ell}\right\|^{2}\left\|\tilde{\boldsymbol{u}}^{\ell}\right\|_{1}^{2}\right] \\
& +\delta_{1} k \sum_{\ell=1}^{M}\left\|\tilde{\boldsymbol{\sigma}}^{\ell}\right\|_{1}^{2} \\
& \leq C\left(\delta_{1}, L\right)\left[\kappa_{1} \kappa_{2}+\kappa_{2}^{2}+k \sum_{\ell=1}^{M}\left\|\tilde{\boldsymbol{\sigma}}^{\ell}\right\|^{2}\left\|\tilde{\boldsymbol{u}}^{\varepsilon, \ell}\right\|_{1}^{2}\right]+\delta_{1} k \sum_{\ell=1}^{M}\left\|\tilde{\boldsymbol{\sigma}}^{\ell}\right\|_{1}^{2}
\end{aligned}
$$

Finally, the estimates obtained for $N L T_{i}\left(\tilde{\sigma}^{\ell}\right), i=1 \ldots 4$ imply

$$
\begin{array}{r}
\sum_{\ell=1}^{M} Q_{\ell}\left(\tilde{\sigma}^{\ell}\right) \leq C\left(\delta_{1}, L, T\right)\left[k \sum_{\ell=1}^{M}\left\|\tilde{\sigma}^{\ell}\right\|^{2}\left\|\tilde{u}^{\varepsilon, \ell}\right\|_{1}^{2}+\left(\kappa_{1} \kappa_{3} k^{2 \eta}+\kappa_{1} \kappa_{2}+\kappa_{2}^{2}\right)\right] \\
+\delta_{1} k \sum_{\ell=1}^{M}\left\|\tilde{\sigma}^{\ell}\right\|_{1}^{2} .
\end{array}
$$

We plug (3.100) into (3.97). We fix $\delta_{1}$ so that $0<\delta_{1}<v$. Since $\omega \in \Omega_{\kappa_{1}} \cap \Omega_{\kappa_{2}} \cap \Omega_{\kappa_{3}}$, we can find a constant $C=C\left(\delta_{1}, L, T\right)>0$ such that

$$
\begin{aligned}
& \max _{1 \leq m \leq M}\left\{\left(\frac{\alpha+1}{2 \alpha}\right)\left\|\sigma^{m}\right\|^{2}+\left(\frac{\alpha-1}{2 \alpha}\right)\left\|\tilde{\sigma}^{m}\right\|^{2}\right\} \\
& +\left(\frac{\alpha-1}{2 \alpha}\right) \sum_{\ell=1}^{M-1}\left\|\tilde{\sigma}^{\ell}-\sigma^{\ell}\right\|^{2}+\left(v-\delta_{1}\right)\left(k \sum_{\ell=1}^{M}\left\|\nabla \tilde{\sigma}^{\ell}\right\|^{2}\right) \\
& +\frac{1}{\varepsilon}\left(k \sum_{\ell=1}^{M}\left\|\operatorname{div} \tilde{\sigma}^{\ell}\right\|^{2}\right) \leq C\left[k \sum_{\ell=1}^{M}\left\|\tilde{\sigma}^{\ell}\right\|^{2}\left\|\tilde{\boldsymbol{u}}^{\varepsilon, \ell}\right\|_{1}^{2}+\left(\kappa_{1} \kappa_{3} k^{2 \eta}+\kappa_{1} \kappa_{2}+\kappa_{2}^{2}+k^{\eta}+\varepsilon\right)\right] .
\end{aligned}
$$


Since we choose $0<\delta_{1}<v$, we have $\left(v-\delta_{1}\right)>0$. In addition, since $\omega \in \Omega_{\kappa_{1}}$, we apply the Gronwall's Lemma we conclude that

$$
\begin{aligned}
\max _{1 \leq m \leq M} & \left\{\left(\frac{\alpha+1}{2 \alpha}\right)\left\|\sigma^{m}\right\|^{2}+\left(\frac{\alpha-1}{2 \alpha}\right)\left\|\tilde{\sigma}^{m}\right\|^{2}\right\} \\
& +k \sum_{\ell=1}^{M}\left\|\nabla \tilde{\sigma}^{\ell}\right\|^{2} \leq C\left(\delta_{1}, L, T\right)\left(\kappa_{1} \kappa_{3} k^{2 \eta}+\kappa_{1} \kappa_{2}+\kappa_{2}^{2}+k^{\eta}+\varepsilon\right) \exp \left(\kappa_{1}\right) .
\end{aligned}
$$

Remember that $\boldsymbol{P}_{\mathbb{H}}$ is stable in $\mathbb{W}^{1,2}$, thus $\left\|\nabla \sigma^{\ell}\right\| \leq C\left\|\nabla \tilde{\boldsymbol{\sigma}}^{\ell}\right\|$.

Lemma 3.7. Under the same assumption as in Lemma 3.6, there exists a constant $C=C(L, T, v)>0$ such that on $\Omega_{\kappa_{1}} \cap \Omega_{\kappa_{2}} \cap \Omega_{\kappa_{3}}$ the error iterates $\left\{\varrho^{\ell}: 1 \leq \ell \leq M\right\}$ of the pressure term in Algorithm 4 satisfies

$$
k \sum_{\ell=1}^{M}\left\|\varrho^{\ell}\right\|^{2} \leq C\left(\kappa_{1} \kappa_{3} k^{2 \eta}+\kappa_{1} \kappa_{2}+\kappa_{2}^{2}+k^{\eta}+\varepsilon\right) \exp \left(\kappa_{1}\right)
$$

Proof. We add (3.94) and (3.93) and get

$$
\begin{gathered}
k\left(\nabla \varrho^{\ell}, \boldsymbol{\varphi}\right)=Q_{\ell}(\boldsymbol{\varphi})+R_{\ell}^{v}(\boldsymbol{\varphi})+\int_{t_{\ell-1}}^{t_{\ell}}\left(\nabla\left(\rho\left(t_{\ell}\right)-\rho(s)\right), \boldsymbol{\varphi}\right) d s \\
-\left(\boldsymbol{\sigma}^{\ell}-\boldsymbol{\sigma}^{\ell-1}, \boldsymbol{\varphi}\right)-k\left(\nabla \tilde{\boldsymbol{\sigma}}^{\ell}, \nabla \boldsymbol{\varphi}\right) .
\end{gathered}
$$

Using the inequality (3.46), we derive that

$$
\begin{aligned}
k^{2} \sum_{\ell=0}^{M}\left\|\varrho^{\ell}\right\|^{2} \leq C \sum_{\ell=0}^{M} \sup _{\boldsymbol{\varphi} \in \mathbb{W}^{1,2}} \frac{1}{\|\boldsymbol{\varphi}\|_{1}^{2}}\left[Q_{\ell}(\boldsymbol{\varphi})+R_{\ell}(\boldsymbol{\varphi})+\int_{t_{\ell-1}}^{t_{\ell}}\left(\nabla\left(\rho\left(t_{\ell}\right)-\rho(s)\right), \boldsymbol{\varphi}\right) d s\right. \\
\left.-\left(\sigma^{\ell}-\sigma^{\ell-1}, \boldsymbol{\varphi}\right)-k\left(\nabla \boldsymbol{\sigma}^{\ell}, \nabla \boldsymbol{\varphi}\right)\right]^{2} .
\end{aligned}
$$

For simplicity let us introduce the following abbreviation

$$
\begin{aligned}
\widetilde{\mathrm{I}} & +\widetilde{\mathrm{II}}+\widetilde{\mathrm{III}}+\widetilde{\mathrm{IV}}+\widetilde{\mathrm{V}} \\
& :=\frac{1}{\|\boldsymbol{\varphi}\|_{1}} Q_{\ell}(\boldsymbol{\varphi})+R_{\ell}(\boldsymbol{\varphi})+\int_{t_{\ell-1}}^{t_{\ell}}\left(\nabla\left(\rho\left(t_{\ell}\right)-\rho(s)\right), \boldsymbol{\varphi}\right) d s-\left(\sigma^{\ell}-\sigma^{\ell-1}, \boldsymbol{\varphi}\right)-k\left(\nabla \sigma^{\ell}, \nabla \boldsymbol{\varphi}\right) .
\end{aligned}
$$

In the following we estimate each term of the right side.

- Term $\widetilde{\mathrm{I}}$ : Here, we get

$$
\begin{aligned}
\widetilde{I} & \leq C \int_{t_{\ell-1}}^{t_{\ell}} \sup _{\boldsymbol{\varphi} \in \mathbb{W}^{1,2}} \frac{1}{\|\boldsymbol{\varphi}\|_{1}}\left(N L T_{1}(\boldsymbol{\varphi})+N L T_{2}(\boldsymbol{\varphi})+N L T_{3}(\boldsymbol{\varphi})+N L T_{4}(\boldsymbol{\varphi})\right) d s, \\
& \leq C \int_{t_{\ell-1}}^{t_{\ell}}\left(\widetilde{N L T}_{1}+\widetilde{N L T}_{2}+\widetilde{N L T}_{3}+\widetilde{N L T}_{4}\right) d s,
\end{aligned}
$$

where with (2.9) and (2.10) we arrive at

$$
\begin{aligned}
& \widetilde{N L T}_{1} \leq C(L) \sup _{\boldsymbol{\varphi} \in \mathbb{W}^{1,2}} \frac{1}{\|\boldsymbol{\varphi}\|_{1}}\|\boldsymbol{u}(s)\|_{1}\|\boldsymbol{\varphi}\|_{1}\left\|\boldsymbol{u}(s)-\boldsymbol{u}\left(t_{\ell}\right)\right\|_{\mathbb{L}^{4}}=\|\boldsymbol{u}(s)\|_{1}\left\|\boldsymbol{u}(s)-\boldsymbol{u}\left(t_{\ell}\right)\right\|_{\mathbb{L}^{4}}, \\
& \widetilde{N L T_{2}} \leq \sup _{\boldsymbol{\varphi} \in \mathbb{W}^{1,2}} \frac{1}{\|\boldsymbol{\varphi}\|_{1}}\left\|\boldsymbol{u}(s)-\boldsymbol{u}\left(t_{\ell}\right)\right\|_{\mathbb{L}^{4}}\left\|\boldsymbol{u}\left(t_{\ell}\right)\right\|_{1}\|\boldsymbol{\varphi}\|_{1}=\left\|\boldsymbol{u}(s)-\boldsymbol{u}\left(t_{\ell}\right)\right\|_{\mathbb{L}^{4}}\left\|\boldsymbol{u}\left(t_{\ell}\right)\right\|_{1}, \\
& \widetilde{N L T_{3}} \leq C(L) \sup _{\boldsymbol{\varphi} \in \mathbb{W}^{1,2}} \frac{1}{\|\boldsymbol{\varphi}\|_{1}}\left\|\boldsymbol{u}\left(t_{\ell}\right)\right\|_{1}\left\|\tilde{\boldsymbol{\epsilon}}^{\ell}\right\|_{1}\|\boldsymbol{\varphi}\|_{1}=\left\|\boldsymbol{u}\left(t_{\ell}\right)\right\|_{1}\left\|\tilde{\boldsymbol{\epsilon}}^{\ell}\right\|_{1}, \\
& \widetilde{N L T}_{4} \leq C(L) \sup _{\boldsymbol{\varphi} \in \mathbb{W}^{1,2}} \frac{1}{\|\boldsymbol{\varphi}\|_{1}}\left\{\left\|\tilde{\boldsymbol{\epsilon}}^{\ell}\right\|_{1}\left\|\boldsymbol{u}\left(t_{\ell}\right)\right\|_{1}\|\boldsymbol{\varphi}\|_{1}+\left\|\tilde{\boldsymbol{\epsilon}}^{\ell}\right\|\left\|\tilde{\boldsymbol{\epsilon}}^{\ell}\right\|_{1}\|\boldsymbol{\varphi}\|_{1}\right\}, \\
& =C(L)\left\{\left\|\tilde{\boldsymbol{\epsilon}}^{\ell}\right\|_{1}\left\|\boldsymbol{u}\left(t_{\ell}\right)\right\|_{1}+\left\|\tilde{\boldsymbol{\epsilon}}^{\ell}\right\|\left\|\tilde{\boldsymbol{\epsilon}}^{\ell}\right\|_{1}\right\} .
\end{aligned}
$$


Integrating gives

$$
\begin{aligned}
& \int_{t_{\ell-1}}^{t_{\ell}} \widetilde{N L T_{1}} d s \leq C(L) \sup _{t_{\ell-1} \leq s \leq t_{\ell}}\|\boldsymbol{u}(s)\|_{1} \int_{t_{\ell-1}}^{t_{\ell}}\left\|\boldsymbol{u}(s)-\boldsymbol{u}\left(t_{\ell}\right)\right\|_{\mathbb{L}^{4}} d s, \\
& \int_{t_{\ell-1}}^{t_{\ell}} \widetilde{N L T_{2}} d s \leq\left\|\boldsymbol{u}\left(t_{\ell}\right)\right\|_{1} \int_{t_{\ell-1}}^{t_{\ell}}\left\|\boldsymbol{u}(s)-\boldsymbol{u}\left(t_{\ell}\right)\right\|_{\mathbb{L}^{4}} d s, \\
& \int_{t_{\ell-1}}^{t_{\ell}} \widetilde{N L T_{3}} d s \leq k\left\|\boldsymbol{u}\left(t_{\ell}\right)\right\|_{1}\left\|\tilde{\boldsymbol{\epsilon}}^{\ell}\right\|_{1}, \\
& \int_{t_{\ell-1}}^{t_{\ell}} \widetilde{N L T_{4}} d s \leq C(L) k\left\{\left\|\tilde{\boldsymbol{e}}^{\ell}\right\|_{1}\left\|\boldsymbol{u}\left(t_{\ell}\right)\right\|_{1}+\left\|\tilde{\boldsymbol{e}}^{\ell}\right\|\left\|\tilde{\boldsymbol{\epsilon}}^{\ell}\right\|_{1}\right\} .
\end{aligned}
$$

From the estimates of $\int_{t_{\ell-1}}^{t_{\ell}} \widetilde{N L T_{i}} d s$, for $i=1, \ldots, 4$, we obtain

$$
\begin{gathered}
\widetilde{\mathrm{I}}^{2} \leq k C(L) \sup _{t_{\ell-1} \leq s \leq t_{\ell}}\|\boldsymbol{u}(s)\|_{1}^{2} \int_{t_{\ell-1}}^{t_{\ell}}\left\|\boldsymbol{u}(s)-\boldsymbol{u}\left(t_{\ell}\right)\right\|_{\mathbb{L}^{4}}^{2} d s+k\left\|\boldsymbol{u}\left(t_{\ell}\right)\right\|_{1}^{2} \int_{t_{\ell-1}}^{t_{\ell}}\left\|\boldsymbol{u}(s)-\boldsymbol{u}\left(t_{\ell}\right)\right\|_{\mathbb{L}^{4}}^{2} d s \\
+k^{2}\left\|\boldsymbol{u}\left(t_{\ell}\right)\right\|_{1}^{2}\left\|\tilde{\boldsymbol{\epsilon}}^{\ell}\right\|_{1}^{2}+C(L) k^{2}\left\{\left\|\tilde{\boldsymbol{\epsilon}}^{\ell}\right\|_{1}^{2}\left\|\boldsymbol{u}\left(t_{\ell}\right)\right\|_{1}^{2}+\left\|\tilde{\boldsymbol{\epsilon}}^{\ell}\right\|^{2}\left\|\tilde{\boldsymbol{e}}^{\ell}\right\|_{1}^{2}\right\} .
\end{gathered}
$$

Summing up for $\ell=1$ to $\ell=M$ gives

$$
\sum_{\ell=1}^{M} \widetilde{\mathrm{I}}^{2} \leq C(L, T) k\left(\kappa_{1} \kappa_{2}+\kappa_{1} \kappa_{3}+\kappa_{2}^{2}\right) .
$$

- Term II: Here, we have

$$
\widetilde{\mathrm{II}}^{2} \leq k \int_{t_{\ell-1}}^{t_{\ell}} \sup _{\boldsymbol{\varphi} \in \mathbb{W}^{1,2}} v^{2} \frac{\| \nabla\left(\boldsymbol{v}\left(t_{\ell}\right)-\boldsymbol{v}(s)\left\|^{2}\right\| \nabla \boldsymbol{\varphi} \|^{2}\right.}{\|\boldsymbol{\varphi}\|_{1}^{2}} d s \leq C k \int_{t_{\ell-1}}^{t_{\ell}} v^{2} \| \nabla\left(\boldsymbol{v}\left(t_{\ell}\right)-\boldsymbol{v}(s) \|^{2} d s .\right.
$$

Then, summing up and using Lemma 2.4 gives

$$
\sum_{\ell=1}^{M} \widetilde{\mathrm{II}}^{2} \leq C(v, T) \leq C(v) k^{\eta+1}
$$

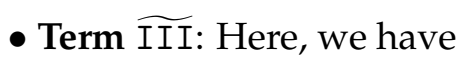

$$
\widetilde{\operatorname{III}}^{2} \leq \sup _{\boldsymbol{\varphi} \in \mathbb{W}^{1,2}} \frac{1}{\|\varphi\|_{1}^{2}} k \int_{t_{\ell-1}}^{t_{\ell}}\left\|\rho\left(t_{\ell}\right)-\rho(s)\right\|^{2}\|\varphi\|_{1}^{2} d s=k \int_{t_{\ell-1}}^{t_{\ell}}\left\|\rho\left(t_{\ell}\right)-\rho(s)\right\|^{2} d s .
$$

Again summing up and using Lemma 2.4 gives

$$
\sum_{\ell=1}^{M} \widetilde{\operatorname{III}}^{2} \leq k \sum_{\ell=1}^{M} \int_{t_{\ell-1}}^{t_{\ell}}\left\|\rho\left(t_{\ell}\right)-\rho(s)\right\|^{2} d s \leq C_{T, 4} k \sum_{\ell=1}^{M} k^{2 \eta+1}=C_{T, 4} k^{2 \eta+1} .
$$

- Term $\widetilde{I V}$ : Here, we proceed in two steps. First, we estimate $\widetilde{I V}$ with a term under a weak norm. Then, we use the Proposition 2.1 to bound this later with terms under $H^{1}$ or $L^{2}$-norm. Thereby, we have

$$
\widetilde{\mathrm{IV}}=\sup _{\boldsymbol{\varphi} \in \mathbb{W}^{1,2}} \frac{1}{\|\boldsymbol{\varphi}\|_{1}}\left(\sigma^{\ell}-\sigma^{\ell-1}, \boldsymbol{\varphi}\right) \leq \sup _{\boldsymbol{\varphi} \in \mathbb{W}^{1,2}} \frac{1}{\|\varphi\|_{1}}\left\|\sigma^{\ell}-\sigma^{\ell-1}\right\|_{-1}\|\varphi\|_{1} \leq\left\|\sigma^{\ell}-\sigma^{\ell-1}\right\|_{-1} .
$$

Next, since $\sigma^{\ell}-\sigma^{\ell-1} \in \mathcal{D}\left(A^{-1}\right)$, we can take $\varphi=A^{-1}\left(\sigma^{\ell}-\sigma^{\ell-1}\right)$ in (3.102), use Proposition 2.1, and arrive at the following estimates:

i) $\left\|\sigma^{\ell}-\sigma^{\ell-1}\right\|_{-1}^{2} \leq C\left(\sigma^{\ell}-\sigma^{\ell-1}, A^{-1}\left(\sigma^{\ell}-\sigma^{\ell-1}\right)\right)$,

ii) $k\left(\nabla \tilde{\sigma}^{\ell}, \nabla A^{-1}\left(\sigma^{\ell}-\sigma^{\ell-1}\right)\right) \leq \delta_{1}\left\|\sigma^{\ell}-\sigma^{\ell-1}\right\|_{-1}^{2}+C \delta_{1} k^{2}\left\|\nabla \tilde{\sigma}^{\ell}\right\|^{2}$, 
iii) $k\left(\nabla \varrho^{\ell}, A^{-1}\left(\sigma^{\ell}-\sigma^{\ell-1}\right)\right)=\int_{t_{\ell-1}}^{t_{\ell}}\left(\nabla\left(\rho\left(t_{\ell}\right)-\rho(s)\right), A^{-1}\left(\sigma^{\ell}-\sigma^{\ell-1}\right)\right) d s=0$,

iv) $R_{\ell}^{v}\left(A^{-1}\left(\sigma^{\ell}-\sigma^{\ell-1}\right)\right) \leq C\left(\nu, \delta_{1}\right) k^{\eta+2}+\delta_{1}\left\|\sigma^{\ell}-\sigma^{\ell-1}\right\|_{-1}^{2}$.

We split the term $Q_{\ell}\left(A^{-1}\left(\sigma^{\ell}-\sigma^{\ell-1}\right)\right)$ as follows

$$
\begin{aligned}
Q_{\ell}\left(A^{-1}\left(\sigma^{\ell}-\sigma^{\ell-1}\right)\right) \leq \int_{t_{\ell-1}}^{t_{\ell}} & N_{L T}\left(A^{-1}\left(\sigma^{\ell}-\sigma^{\ell-1}\right)\right)+\operatorname{NLT}_{2}\left(A^{-1}\left(\sigma^{\ell}-\sigma^{\ell-1}\right)\right) \\
& +\operatorname{NLT}_{3}\left(A^{-1}\left(\sigma^{\ell}-\sigma^{\ell-1}\right)\right)+\operatorname{NLT}_{4}\left(A^{-1}\left(\sigma^{\ell}-\sigma^{\ell-1}\right)\right) d s,
\end{aligned}
$$

where each of terms $\operatorname{NLT}_{j}\left(A^{-1}\left(\sigma^{\ell}-\sigma^{\ell-1}\right)\right)$ for $j=1,2,3,4$, are estimated as follows:

$$
\begin{array}{r}
\operatorname{NLT}_{1}\left(A^{-1}\left(\boldsymbol{\sigma}^{\ell}-\boldsymbol{\sigma}^{\ell-1}\right)\right) \leq C_{\delta_{1}}(L) k\|\boldsymbol{u}(s)\|_{1}^{2}\left\|\boldsymbol{u}(s)-\boldsymbol{u}\left(t_{\ell}\right)\right\|_{\mathbb{L}^{4}}^{2}+\frac{\delta_{1}}{k}\left\|A^{-1}\left(\boldsymbol{\sigma}^{\ell}-\sigma^{\ell-1}\right)\right\|_{1}^{2}, \\
\operatorname{NLT}_{2}\left(\boldsymbol{A}^{-1}\left(\boldsymbol{\sigma}^{\ell}-\boldsymbol{\sigma}^{\ell-1}\right)\right) \leq C_{\delta_{1}} k\left\|\boldsymbol{u}(s)-\boldsymbol{u}\left(t_{\ell}\right)\right\|_{\mathbb{L}^{4}}^{2}\left\|\boldsymbol{u}\left(t_{\ell}\right)\right\|_{1}^{2}+\frac{\delta_{1}}{k}\left\|A^{-1}\left(\boldsymbol{\sigma}^{\ell}-\sigma^{\ell-1}\right)\right\|_{1}^{2}, \\
\operatorname{NLT}_{3}\left(\boldsymbol{A}^{-1}\left(\boldsymbol{\sigma}^{\ell}-\boldsymbol{\sigma}^{\ell-1}\right)\right) \leq C\left(\delta_{1}, L\right) k\left\|\boldsymbol{u}\left(t_{\ell}\right)\right\|_{1}^{2}\left\|\tilde{\boldsymbol{\epsilon}}^{\ell}\right\|_{1}^{2}+\frac{\delta_{1}}{k}\left\|A^{-1}\left(\boldsymbol{\sigma}^{\ell}-\sigma^{\ell-1}\right)\right\|_{1}^{2}, \\
\operatorname{NLT}_{4}\left(\boldsymbol{A}^{-1}\left(\boldsymbol{\sigma}^{\ell}-\sigma^{\ell-1}\right)\right) \leq C\left(\delta_{1}, L\right) k\left\{\left\|\tilde{\boldsymbol{\epsilon}}^{\ell}\right\|_{1}^{2}\left\|\boldsymbol{u}\left(t_{\ell}\right)\right\|_{1}^{2}+\left\|\tilde{\boldsymbol{\epsilon}}^{\ell}\right\|^{2}\left\|\tilde{\boldsymbol{\epsilon}}^{\ell}\right\|_{1}^{2}+\left\|\tilde{\boldsymbol{\sigma}}^{\ell}\right\|^{2}\left\|\tilde{\boldsymbol{u}}^{\varepsilon, \ell}\right\|_{1}^{2}\right\} \\
+\frac{2 \delta_{1}}{k}\left\|\boldsymbol{A}^{-1}\left(\boldsymbol{\sigma}^{\ell}-\boldsymbol{\sigma}^{\ell-1}\right)\right\|_{1}^{2} .
\end{array}
$$

All together, the estimates of $N L T_{i}\left(A^{-1}\left(\sigma^{\ell}-\sigma^{\ell-1}\right)\right)$, for $i=1, \ldots, 4$, lead to

$$
\begin{aligned}
Q_{\ell}\left(A^{-1}\left(\sigma^{\ell}-\sigma^{\ell-1}\right)\right) \leq & C\left(\delta_{1}, L\right) k \int_{t_{\ell-1}}^{t_{\ell}}\left\|\boldsymbol{u}(s)-\boldsymbol{u}\left(t_{\ell}\right)\right\|_{\mathbb{L}^{4}}^{2}\left[\|\boldsymbol{u}(s)\|_{1}^{2}+\left\|\boldsymbol{u}\left(t_{\ell}\right)\right\|_{1}^{2}\right] d s \\
& +C\left(\delta_{1}, L\right) k^{2}\left\{\left\|\tilde{\boldsymbol{\epsilon}}^{\ell}\right\|_{1}^{2}\left\|\boldsymbol{u}\left(t_{\ell}\right)\right\|_{1}^{2}+\left\|\tilde{\boldsymbol{\epsilon}}^{\ell}\right\|^{2}\left\|\tilde{\boldsymbol{\epsilon}}^{\ell}\right\|_{1}^{2}+\left\|\tilde{\boldsymbol{\sigma}}^{\ell}\right\|^{2}\left\|\tilde{\boldsymbol{u}}^{\varepsilon, \ell}\right\|_{1}^{2}\right\} \\
& +C\left(\delta_{1}, L\right) k^{2}\left\|\boldsymbol{u}\left(t_{\ell}\right)\right\|_{1}^{2}\left\|\tilde{\boldsymbol{\epsilon}}^{\ell}\right\|_{1}^{2}+4 \delta_{1}\left\|\boldsymbol{A}^{-1}\left(\boldsymbol{\sigma}^{\ell}-\boldsymbol{\sigma}^{\ell-1}\right)\right\|_{1}^{2} .
\end{aligned}
$$

In addition on $\Omega_{\kappa_{3}}$ we have

$$
\begin{aligned}
Q_{\ell}\left(\boldsymbol{A}^{-1}\left(\boldsymbol{\sigma}^{\ell}-\boldsymbol{\sigma}^{\ell-1}\right)\right) \leq & C\left(\delta_{1}, L\right)\left(\sup _{t_{\ell-1} \leq s \leq t_{\ell}}\|\boldsymbol{u}(s)\|_{1}^{2}+C\left(\delta_{1}\right)\left\|\boldsymbol{u}\left(t_{\ell}\right)\right\|_{1}^{2}\right) \kappa_{3} k^{2 \eta+2} \\
& +C\left(\delta_{1}, L\right) k^{2}\left\{\left\|\tilde{\boldsymbol{\epsilon}}^{\ell}\right\|_{1}^{2}\left\|\boldsymbol{u}\left(t_{\ell}\right)\right\|_{1}^{2}+\left\|\tilde{\boldsymbol{\epsilon}}^{\ell}\right\|^{2}\left\|\tilde{\boldsymbol{\epsilon}}^{\ell}\right\|_{1}^{2}+\left\|\tilde{\boldsymbol{\sigma}}^{\ell}\right\|^{2}\left\|\tilde{\boldsymbol{u}}^{\varepsilon, \ell}\right\|_{1}^{2}\right\} \\
& +C\left(\delta_{1}, L\right) k^{2}\left\|\boldsymbol{u}\left(t_{\ell}\right)\right\|_{1}^{2}\left\|\tilde{\boldsymbol{\epsilon}}^{\ell}\right\|_{1}^{2}+4 \delta_{1}\left\|\boldsymbol{\sigma}^{\ell}-\sigma^{\ell-1}\right\|_{-1}^{2} .
\end{aligned}
$$

Now summing for $\ell=1$ to $\ell=M$, we have

$$
\begin{aligned}
\sum_{\ell=1}^{M} Q_{\ell}\left(A^{-1}\left(\sigma^{\ell}-\sigma^{\ell-1}\right)\right) \leq C\left(\delta_{1}, L\right)\left(\sup _{0 \leq s \leq T}\|\boldsymbol{u}(s)\|_{1}^{2}+\max _{1 \leq \ell \leq M}\left\|\boldsymbol{u}\left(t_{\ell}\right)\right\|_{1}^{2}\right) \kappa_{3} k^{2 \eta+2} \\
+C\left(\delta_{1}, L\right) k \max _{1 \leq \ell \leq M}\left\|\boldsymbol{u}\left(t_{\ell}\right)\right\|_{1}^{2}\left(k \sum_{\ell=1}^{M}\left\|\tilde{\boldsymbol{\epsilon}}^{\ell}\right\|_{1}^{2}\right)+k \max _{1 \leq \ell \leq M}\left\|\tilde{\boldsymbol{\epsilon}}^{\ell}\right\|^{2}\left(k \sum_{\ell=1}^{M}\left\|\tilde{\boldsymbol{\epsilon}}^{\ell}\right\|_{1}^{2}\right) \\
+k \max _{1 \leq \ell \leq M}\left\|\tilde{\boldsymbol{\sigma}}^{\ell}\right\|^{2}\left(k \sum_{\ell=1}^{M}\left\|\tilde{\boldsymbol{u}}^{\varepsilon, \ell}\right\|_{1}^{2}\right)+4 \delta_{1} \sum_{\ell=1}^{M}\left\|\sigma^{\ell}-\sigma^{\ell-1}\right\|_{-1}^{2} .
\end{aligned}
$$

Since we have due to the assumptions $\omega \in \Omega_{\mathcal{K}_{1}} \cap \Omega_{\mathcal{K}_{2}} \cap \Omega_{\mathcal{K}_{3}}$ we obtain using (3.96)

$$
\begin{aligned}
\sum_{\ell=1}^{M} Q_{\ell}\left(A^{-1}\left(\sigma^{\ell}-\sigma^{\ell-1}\right)\right) & \leq C\left(\delta_{1}, L\right) k\left(\kappa_{1} \kappa_{3} k^{2 \eta+1}+\kappa_{1} \kappa_{2}+\kappa_{2}^{2}\right)+4 \delta_{1} \sum_{\ell=1}^{M}\left\|\sigma^{\ell}-\sigma^{\ell-1}\right\|_{-1}^{2} \\
& +C\left(\delta_{1}, L, T\right) k\left(\kappa_{1} \kappa_{3} k^{2 \eta}+\kappa_{1} \kappa_{2}+\kappa_{2}^{2}+k^{\eta}+\varepsilon\right) \exp \left(\kappa_{1}\right) .
\end{aligned}
$$


All together we obtain,

$$
\begin{aligned}
\sum_{\ell=1}^{M}\left\|\sigma^{\ell}-\sigma^{\ell-1}\right\|_{-1}^{2} & \leq 6 \delta_{1} \sum_{\ell=1}^{M}\left\|\sigma^{\ell}-\sigma^{\ell-1}\right\|_{-1}^{2}+C\left(\delta_{1}\right) k^{2} \sum_{\ell=1}^{M}\left\|\nabla \tilde{\sigma}^{\ell}\right\|^{2}+C\left(\nu, \delta_{1}\right) k^{\eta+1} \\
& +C\left(\delta_{1}, L, T\right) k\left(\kappa_{1} \kappa_{3} k^{2 \eta}+\kappa_{1} \kappa_{2}+\kappa_{2}^{2}+k^{\eta}+\varepsilon\right) \exp \left(\kappa_{1}\right) .
\end{aligned}
$$

The terms with $\left\|\sigma^{\ell}-\sigma^{\ell-1}\right\|_{-1}^{2}$ are absorbed by the left hand side. Thanks to (3.96),

$$
\left(1-6 \delta_{1}\right) \sum_{\ell=1}^{M}\left\|\sigma^{\ell}-\sigma^{\ell-1}\right\|_{-1}^{2} \leq C\left(\delta_{1}, L, T\right) k\left(\kappa_{1} \kappa_{3} k^{2 \eta}+\kappa_{1} \kappa_{2}+\kappa_{2}^{2}+k^{\eta}+\varepsilon\right) \exp \left(\kappa_{1}\right) .
$$

We can choose $\delta_{1}$ so that $\left(1-6 \delta_{1}\right)>0$. Note that $1 \leq \exp (x)$ for all $x \in \mathbb{R}$. Therefore,

$$
\sum_{\ell=1}^{M} \widetilde{\mathrm{IV}}^{2} \leq C(L, T) k\left(\kappa_{1} \kappa_{3} k^{2 \eta}+\kappa_{1} \kappa_{2}+\kappa_{2}^{2}+k^{\eta}+\varepsilon\right) \exp \left(\kappa_{1}\right)
$$

- Term $\widetilde{\mathrm{V}}$ : Here, we have

$$
\widetilde{\mathrm{V}}=\sup _{\boldsymbol{\varphi}_{\ell} \in \mathbb{W}^{1,2}} \frac{1}{\|\boldsymbol{\varphi}\|_{1}} k\left(\nabla \tilde{\boldsymbol{\sigma}}^{\ell}, \nabla \boldsymbol{\varphi}\right) \leq \sup _{\boldsymbol{\varphi} \in \mathbb{W}^{1,2}} \frac{1}{\|\boldsymbol{\varphi}\|_{1}} k\left\|\nabla \tilde{\sigma}^{\ell}\right\|\|\nabla \boldsymbol{\varphi}\|=C k\left\|\nabla \tilde{\sigma}^{\ell}\right\| .
$$

Summing up and using (3.96) gives

$$
\sum_{\ell=1}^{M} \widetilde{\mathrm{V}}^{2} \leq C k^{2} \sum_{\ell=1}^{M}\left\|\nabla \tilde{\sigma}^{\ell}\right\|^{2} \leq C\left(\delta_{1}, L, T\right) k\left(\kappa_{1} \kappa_{3} k^{2 \eta}+\kappa_{1} \kappa_{2}+\kappa_{2}^{2}+k^{\eta}+\varepsilon\right) \exp \left(\kappa_{1}\right) .
$$

Collecting $\widetilde{\mathrm{I}}, \mathrm{II}, \widetilde{\mathrm{III}}, \widetilde{\mathrm{IV}}$, and $\widetilde{\mathrm{V}}$, we obtain

$$
\begin{aligned}
k^{2} \sum_{\ell=0}^{M}\left\|\varrho^{\ell}\right\|^{2} \leq \sum_{\ell=0}^{M} & \{\widetilde{\mathrm{I}}+\widetilde{\mathrm{II}}+\widetilde{\mathrm{III}}+\widetilde{\mathrm{IV}}+\widetilde{\mathrm{V}}\}^{2} \\
\leq C & (L, T) k\left(\kappa_{1} \kappa_{2}+\kappa_{1} \kappa_{3}+\kappa_{2}^{2}\right)+C(v, T) k^{\eta+1}+C_{T, 4} k^{2 \eta+1} \\
& +C(L, T) k\left(\kappa_{1} \kappa_{3} k^{2 \eta}+\kappa_{1} \kappa_{2}+\kappa_{2}^{2}+k^{\eta}+\varepsilon\right) \exp \left(\kappa_{1}\right) \\
& +C(L, T) k\left(\kappa_{1} \kappa_{3} k^{2 \eta}+\kappa_{1} \kappa_{2}+\kappa_{2}^{2}+k^{\eta}+\varepsilon\right) \exp \left(\kappa_{1}\right) .
\end{aligned}
$$

Because $1<\exp (x)$ for all $x \in \mathbb{R}$ and with a limiting order term $\left(k^{\eta}\right)$, we have

$$
k \sum_{\ell=0}^{M}\left\|\varrho^{\ell}\right\|^{2} \leq C(L, T, \nu)\left(\kappa_{1} \kappa_{3} k^{2 \eta}+\kappa_{1} \kappa_{2}+\kappa_{2}^{2}+k^{\eta}+\varepsilon\right) \exp \left(\kappa_{1}\right) .
$$

\section{MAIN RESUltS}

Let us define the errors $\mathbf{e}^{\ell}=\boldsymbol{u}\left(t_{\ell}\right)-\boldsymbol{u}^{\varepsilon, \ell}$ and $\mathrm{q}^{\ell}=\mathrm{p}\left(t_{\ell}\right)-\mathrm{p}^{\varepsilon, \ell}$. Here in the final section, we use the estimates of the iterates $\left\{\boldsymbol{\epsilon}^{\ell}, \omega^{\ell}\right\}_{\ell}$ and $\left\{\boldsymbol{\sigma}^{\ell}, \varrho^{\ell}\right\}_{\ell}$ to derive an estimate for $\left\{\mathbf{e}^{\ell}, q^{\ell}\right\}_{\ell}$, show convergence in probability of Algorithm 1, and deduce from that strong convergence.

We set

$$
\begin{aligned}
& \mathcal{E}^{M}:=\max _{1 \leq m \leq M}\left\|\mathbf{e}^{m}\right\|^{2}+v k \sum_{\ell=1}^{M}\left\|\nabla \mathbf{e}^{\ell}\right\|^{2}+k \sum_{\ell=1}^{M}\left\|\mathbf{q}^{\ell}\right\|^{2}, \\
& \widetilde{\mathcal{E}}^{M}:=\max _{1 \leq m \leq M}\left\|\mathbf{e}^{m}\right\|^{2}+\left(v k \sum_{\ell=1}^{M}\left\|\nabla \mathbf{e}^{\ell}\right\|^{2}\right)^{1 / 2}+\left(k \sum_{\ell=1}^{M}\left\|\mathbf{q}^{\ell}\right\|^{2}\right)^{1 / 2}, \\
& \mathcal{E}_{1}^{M}:=\max _{1 \leq m \leq M}\left\|\boldsymbol{\epsilon}^{m}\right\|^{2}+v k \sum_{\ell=1}^{M}\left\|\nabla \boldsymbol{\epsilon}^{\ell}\right\|^{2}+k \sum_{\ell=1}^{M}\left\|\omega^{\ell}\right\|^{2}, \\
& \mathcal{E}_{2}^{M}:=\max _{1 \leq m \leq M}\left\|\sigma^{m}\right\|^{2}+v k \sum_{\ell=1}^{M}\left\|\nabla \sigma^{\ell}\right\|^{2}+k \sum_{\ell=1}^{M}\left\|\varrho^{\ell}\right\|^{2} .
\end{aligned}
$$


Theorem 4.1. Let $\mathcal{E}^{M}$ be defined in (4.103). If $\varepsilon=\kappa^{\eta}$, the Algorithm 1 converges in probability with order $0<r<\eta$. In particular, we have

$$
\lim _{\widetilde{C} \rightarrow \infty} \lim _{k \rightarrow 0} \mathbb{P}\left[\mathcal{E}^{M} \geq \widetilde{C} k^{r}\right]=0
$$

Proof. Let $\widetilde{C}, r>0$ be some arbitrary constants which will be fixed at the end of the proof. By the Chebyshev inequality

$$
\begin{aligned}
& \mathbb{P}\left[\mathcal{E}^{M} \geq \widetilde{C} k^{r}\right] \leq \mathbb{P}\left(\Omega \backslash \Omega_{\kappa_{1}}\right)+\mathbb{P}\left(\Omega \backslash \Omega_{\kappa_{2}}\right)+\mathbb{P}\left(\Omega \backslash \Omega_{\kappa_{3}}\right)+\mathbb{P}\left[\mathcal{E}^{M} \geq \widetilde{C} k^{r} \mid \Omega_{\kappa_{1}} \cap \Omega_{\kappa_{2}} \cap \Omega_{\kappa_{3}}\right] \\
& \leq \frac{1}{\kappa_{1}} \mathbb{E}\left[\sup _{0 \leq s \leq T}\|\boldsymbol{u}(s)\|_{\mathbb{V}}^{2}+v k \sum_{\ell=1}^{M}\left\|\boldsymbol{u}^{\ell}\right\|_{1}^{2}\right]+\frac{1}{\kappa_{2}} \mathbb{E}\left[\max _{1 \leq \ell \leq M}\left\|\boldsymbol{\epsilon}^{\ell}\right\|^{2}+v k \sum_{\ell=1}^{M}\left\|\boldsymbol{\epsilon}^{\ell}\right\|_{1}^{2}+k \sum_{\ell=1}^{M}\left\|\boldsymbol{\omega}^{\ell}\right\|^{2}\right] \\
& \quad+\frac{1}{\kappa_{3}|t-s|^{2 \eta}} \mathbb{E}\left[\|\boldsymbol{u}(s)-\boldsymbol{u}(t)\|_{\mathbb{L}^{4}}^{2}\right]+\frac{\mathbb{E}\left[\mathcal{E}^{M} \mid \Omega_{\kappa_{1}} \cap \Omega_{\kappa_{2}} \cap \Omega_{\kappa_{3}}\right]}{\widetilde{C} k^{r}} .
\end{aligned}
$$

Observe, that we can write $\mathbf{e}^{\ell}=\epsilon^{\ell}+\sigma^{\ell}$ and $\mathrm{q}^{\ell}=\omega^{\ell}+\varrho^{\ell}$. Now, it follows by the definition of $\Omega_{\mathcal{K}_{2}}$ (see (3.95)), by Lemma 3.6, and Lemma 3.7

$$
\begin{aligned}
\mathbb{E}\left[\mathcal{E}^{M} \mid \Omega_{\kappa_{1}} \cap \Omega_{\kappa_{2}} \cap \Omega_{\kappa_{3}}\right] & \leq \mathbb{E}\left[\mathcal{E}_{1}^{M} \mid \Omega_{\kappa_{1}} \cap \Omega_{\kappa_{2}} \cap \Omega_{\kappa_{3}}\right]+\mathbb{E}\left[\mathcal{E}_{2}^{M} \mid \Omega_{\kappa_{1}} \cap \Omega_{\kappa_{2}} \cap \Omega_{\kappa_{3}}\right] \\
& \leq \kappa_{2}+C\left(\kappa_{1} \kappa_{3} k^{2 \eta}+\kappa_{1} \kappa_{2}+\kappa_{2}^{2}+k^{\eta}+\varepsilon\right) \exp \left(\kappa_{1}\right),
\end{aligned}
$$

where $\mathcal{E}_{1}^{M}$ and $\mathcal{E}_{2}^{M}$ are defined by (4.105) and (4.106) respectively. Moreover, by estimate (2.14), Lemma 3.2, and Lemma 3.4 we obtain

$$
\begin{aligned}
\mathbb{P}\left[\mathcal{E}^{M} \geq \widetilde{C} k^{r}\right] & \leq \frac{\kappa_{2}+C\left(\kappa_{1} \kappa_{3} k^{2 \eta}+\kappa_{1} \kappa_{2}+\kappa_{2}^{2}+k^{\eta}+\varepsilon\right) \exp \left(\kappa_{1}\right)}{\widetilde{C} k^{r}}+\frac{C}{\kappa_{1}}+\frac{C\left(k^{\eta}+\varepsilon\right)}{\kappa_{2}}+\frac{C}{\kappa_{3}} \\
& \leq \frac{C\left(\kappa_{2}+\kappa_{3} k^{2 \eta}+\kappa_{2}^{2}+k^{\eta}+\varepsilon\right) \exp \left(2 \kappa_{1}\right)}{\widetilde{C} k^{r}}+\frac{C}{\kappa_{1}}+\frac{C\left(k^{\eta}+\varepsilon\right)}{\kappa_{2}}+\frac{C}{\kappa_{3}} .
\end{aligned}
$$

Let $\mu>0$. We fix $\varepsilon=k^{\eta}, \kappa_{1}=\ln k^{-\mu / 2}, \kappa_{2}=k^{\mu+r}$, and $\kappa_{3}=k^{-\eta}$ with $k<1$. Therefore, we have

$$
\mathbb{P}\left[\mathcal{E}^{M} \geq \widetilde{C} k^{r}\right] \leq \frac{C\left(k^{r}+k^{\eta-\mu}\right)}{\widetilde{C} k^{r}}-\frac{C}{\ln k^{\mu}}+C k^{\eta-\mu-r}+C k^{\eta} .
$$

Let us remind, that we fixed the constant $r$ in the beginning, such that $\eta-\mu-r>0$. Now, we are ready to go to the limit:

$$
\lim _{\widetilde{C} \rightarrow \infty k \rightarrow 0} \lim _{\mathbb{P}}\left[\mathcal{E}^{M} \geq \widetilde{C} k^{r}\right] \leq \lim _{\widetilde{C} \rightarrow \infty} \lim _{k \rightarrow 0}\left(\frac{C}{\widetilde{C}}-\frac{C}{\ln k^{\mu}}+C k^{\eta-\mu-r}+C k^{\eta}\right)=\lim _{\widetilde{C} \rightarrow \infty} \frac{C}{\widetilde{C}}=0 .
$$

This gives the assertion.

A consequence of this theorem is strong convergence of iterates of the scheme. This will be shown by the following corollary.

Corollary 4.2. Let $\widetilde{\mathcal{E}}^{M}$ be defined as in (4.104). Under the assumption of Theorem 4.1 we have

$$
\lim _{M \rightarrow \infty} \mathbb{E}\left[\widetilde{\mathcal{E}}^{M}\right]=0 .
$$

Proof. Let $\widetilde{C}>0$ an arbitrary constant. We define the sample set

$$
\Omega_{\widetilde{C}, k}:=\left\{\mathcal{E}^{M} \geq \widetilde{C} k^{r}\right\} .
$$

From the law of total probability we deduce that

$$
\mathbb{E}\left[\widetilde{\mathcal{E}}^{M}\right]=\mathbb{E}\left[\widetilde{\mathcal{E}}^{M} \mid \Omega_{\widetilde{C}, k}\right] \mathbb{P}\left(\Omega_{\widetilde{C}, k}\right)+\mathbb{E}\left[\widetilde{\mathcal{E}}^{M} \mid \Omega \backslash \Omega_{\widetilde{C}, k}\right] \mathbb{P}\left(\Omega \backslash \Omega_{\widetilde{C}, k}\right) .
$$

Since $\mathbb{P}\left(\Omega \backslash \Omega_{\widetilde{C}, k}\right) \leq 1$, and by definition of $\Omega_{\widetilde{C}, k^{\prime}}$

$$
\mathbb{E}\left[\widetilde{\mathcal{E}}^{M}\right] \leq \mathbb{E}\left[\widetilde{\mathcal{E}}^{M} \mid \Omega_{\widetilde{C}, k}\right] \mathbb{P}\left(\Omega_{\widetilde{C}, k}\right)+\widetilde{C} k^{r / 2} .
$$


Using the definition of conditional expectation and the Cauchy-Schwartz inequality we obtain

$$
\mathbb{E}\left[\widetilde{\mathcal{E}}^{M} \mid \Omega_{\widetilde{C}, k}\right] \mathbb{P}\left(\Omega_{\widetilde{C}, k}\right) \leq \mathbb{E}\left[\left(\widetilde{\mathcal{E}}^{M}\right)^{2}\right]\left(\mathbb{P}\left(\Omega_{\widetilde{C}, k}\right)\right)^{1 / 2}
$$

Remember that $\mathbf{e}^{\ell}=\boldsymbol{u}\left(t_{\ell}\right)-\boldsymbol{u}^{\varepsilon, \ell}$ and $\mathbf{e}^{\ell}=\mathrm{p}\left(t_{\ell}\right)-\mathrm{p}^{\varepsilon, \ell}$. Using now Eq. (2.12), Lemma 3.2(iii), Eq. (2.13), Proposition 2.3, and Lemma 3.3, we arrive at

$$
\begin{aligned}
& \mathbb{E}\left[\left(\widetilde{\mathcal{E}}^{M}\right)^{2}\right] \leq \mathbb{E}\left[\max _{1 \leq m \leq M}\left\|\boldsymbol{u}\left(t_{m}\right)\right\|^{4}\right]+\mathbb{E}\left[\max _{1 \leq m \leq M}\left\|\boldsymbol{u}^{\varepsilon, m}\right\|^{4}\right]+\mathbb{E}\left(v k \sum_{\ell=1}^{M}\left\|\nabla \boldsymbol{u}\left(t_{\ell}\right)\right\|^{2}\right) \\
& +\mathbb{E}\left(v k \sum_{\ell=1}^{M}\left\|\nabla \boldsymbol{u}^{\varepsilon, \ell}\right\|^{2}\right)+\mathbb{E}\left(k \sum_{\ell=1}^{M}\left\|\mathrm{p}\left(t_{\ell}\right)\right\|^{2}\right)+\mathbb{E}\left(k \sum_{\ell=1}^{M}\left\|\mathrm{p}^{\varepsilon, \ell}\right\|^{2}\right) \leq C\left(T, L, \boldsymbol{u}^{0}, v\right) .
\end{aligned}
$$

Consequently, we get

$$
\mathbb{E}\left[\widetilde{\mathcal{E}}^{M}\right] \leq C\left(T, L, u^{0}, v\right)\left(\mathbb{P}\left(\Omega_{\widetilde{C}, k}\right)\right)^{1 / 2}+\widetilde{C} k^{r / 2}
$$

Now we fix $\widetilde{C}=k^{-r / 4}$ from the beginning and define $\widetilde{\Omega}_{M}:=\Omega_{M^{r / 4}, M^{-1}}$. To conclude, we take the limit for $M \rightarrow \infty$ and apply Theorem 4.1,

$$
\lim _{M \rightarrow \infty} \mathbb{E}\left[\widetilde{\mathcal{E}}^{M}\right] \leq C\left(T, L, \boldsymbol{u}^{0}, v\right)\left(\lim _{M \rightarrow \infty} \mathbb{P}\left(\widetilde{\Omega}_{M}\right)\right)^{1 / 2}+\lim _{M \rightarrow \infty} \frac{1}{M^{r / 4}}=0 .
$$

This gives the assertion.

\section{REFERENCES}

[1] I. Babuška. The finite element method with Lagrangian multipliers. Numer. Math., 20(3):179-192, Jun 1973.

[2] Ľ. Baňas, Z. Brzeźniak, M. Neklyudov, and A. Prohl. A convergent finite-element-based discretization of the stochastic Landau-Lifshitz-Gilbert equation. IMA J. Numer. Anal., 34(2):502, 2014.

[3] P. Bernard and J. Wallace. Turbulent Flow: Analysis, Measurement, and Prediction. John Wiley \& Sons, 2002.

[4] H. Bessaih, Z. Brzeźniak, and A. Millet. Splitting up method for the 2D stochastic Navier-Stokes equations. Stoch. Partial Differ. Equ., Anal. Comput., 2(4):433-470, 2014.

[5] B. Birnir. Turbulence of a unidirectional flow. In Probability, Geometry and Integrable Systems For Henry McKean's SeventyFifth Birthday, pages 29-52. Cambridge University Press, 2008.

[6] B. Birnir. The Kolmogorov-Obukhov statistical theory of turbulence. J. Nonlinear Sci., 23(4):657-688, 2013.

[7] F. Boyer and P. Fabrie. Mathematical tools for the study of the incompressible Navier-Stokes equations and related models, volume 183. Springer Science \& Business Media, 2012.

[8] F. Brezzi. On the existence, uniqueness and approximation of saddle-point problems arising from Lagrangian multipliers. ESAIM-Math. Model. Numer. Anal.-Model. Math. Anal. Numer., 8(R2):129-151, 1974.

[9] Z. Brzeźniak, E. Carelli, and A. Prohl. Finite-element-based discretizations of the incompressible Navier-Stokes equations with multiplicative random forcing. IMA J. Numer. Anal., 33(3):771-824, 2013.

[10] Z. Brzeźniak and B. Ferrario. A note on stochastic Navier-Stokes equations with not regular multiplicative noise. Stoch. Partial Differ. Equ., Anal. Comput., pages 1-28, 2016.

[11] M. Capiński and S. Peszat. On the existence of a solution to stochastic Navier-Stokes equations. Nonlinear Anal.-Theory Methods Appl., 44(2):141-177, 2001.

[12] E. Carelli, E. Hausenblas, and A. Prohl. Time-splitting methods to solve the stochastic incompressible Stokes equation. SIAM J. Numer. Anal., 50(6):2917-2939, 2012.

[13] E. Carelli and A. Prohl. Rates of convergence for discretizations of the stochastic incompressible Navier-Stokes equations. SIAM J. Numer. Anal., 50(5):2467-2496, 2012.

[14] L. Cattabriga. Su un problema al contorno relativo al sistema di equazioni di Stokes. Rend. Semin. Mat. Univ. Padova, 31:308-340, 1961.

[15] R. Courant. Variational methods for the solution of problems of equilibrium and vibrations. Bull. Amer. Math. Soc., 49(1):1-23, 011943.

[16] A. de Bouard and A. Debussche. A semi-discrete scheme for the stochastic nonlinear Schrödinger equation. Numer. Math., 96(4):733-770, 2004.

[17] A. Debussche and J. Printems. Convergence of a semi-discrete scheme for the stochastic Korteweg-de Vries equation. Discrete Contin. Dyn. Syst.-Ser. B, 6(4):761-781, 2006.

[18] B. P. W. Fernando, B. Rüdiger, and S. S. Sritharan. Mild solutions of stochastic Navier-Stokes equation with jump noise in $L^{p}$-spaces. Math. Nachr., 288(14-15):1615-1621, 2015.

[19] F. Flandoli. Dissipativity and invariant measures for stochastic Navier-Stokes equations. NoDea-Nonlinear Differ. Equ. Appl., 1(4):403-423, 1994. 
[20] F. Flandoli and D. Gatarek. Martingale and stationary solutions for stochastic Navier-Stokes equations. Probab. Theory Relat. Field, 102(3):367-391, 1995.

[21] F. Flandoli and B. Schmalfuß. Weak solutions and attractors for three-dimensional Navier-Stokes equations with nonregular force. J. Dyn. Differ. Equ., 11(2):355-398, 1999.

[22] F. Flandoli and V. M. Tortorelli. Time discretization of Ornstein-Uhlenbeck equations and stochastic Navier-Stokes equations with a generalized noise. Stochastics, 55(1-2):141-165, 1995.

[23] Y. Giga and T. Miyakawa. Solutions in $L^{r}$ of the Navier-Stokes initial value problem. Arch. Ration. Mech. Anal., 89(3):267-281, 1985.

[24] J.-L. Guermond, P. D. Minev, and J. Shen. An overview of projection methods for incompressible flows. Comput. Meth. Appl. Mech. Eng., 195(44-47):6011 - 6045, 2006.

[25] T. Y. Hou, W. Luo, B. Rozovskii, and H.-M. Zhou. Wiener chaos expansions and numerical solutions of randomly forced equations of fluid mechanics. J. Comput. Phys., 216(2):687 - 706, 2006.

[26] I. Karatzas and S. Shreve. Brownian Motion and Stochastic Calculus. Graduate Texts in Mathematics. Springer New York, 2014.

[27] J. A. Langa, J. Real, and J. Simon. Existence and regularity of the pressure for the stochastic Navier-Stokes equations. Appl. Math. Optim., 48(3):195-210, 2003.

[28] J.-L. Lions. Quelques méthodes de résolution des problemes aux limites non linéaires, volume 31. Dunod Paris, 1969.

[29] U. Manna, J.-L. Menaldi, and S. S. Sritharan. Stochastic 2-D Navier-Stokes equation with artificial compressibility. Commun. Stoch. Anal., 1(1):123-139, 2007.

[30] G. N. Milstein and M. Tretyakov. Layer methods for stochastic navier-stokes equations using simplest characteristics. J. Comput. Appl. Math., 302:1 - 23, 2016.

[31] J. Nečas. Direct methods in the theory of elliptic equations. Springer Science \& Business Media, 2011.

[32] S. Pope. Turbulent Flows. Cambridge University Press, 2000.

[33] J. Printems. On the discretization in time of parabolic stochastic partial differential equations. ESAIM-Math. Model. Numer. Anal.-Model. Math. Anal. Numer., 35(6):1055-1078, 2001.

[34] A. Prohl. Projection and Quasi-Compressibility Methods for Solving the Incompressible Navier-Stokes Equations. Advances in Numerical Mathematics. Vieweg+Teubner Verlag, 2013.

[35] J. Shen. On error estimates of projection methods for Navier-Stokes equations: First-order schemes. SIAM J. Numer. Anal., 29(1):57-77, 1992.

[36] J. Shen. On error estimates of some higher order projection and penalty-projection methods for Navier-Stokes equations. Numer. Math., 62(1):49-73, 1992.

[37] J. Shen. On error estimates of the penalty method for unsteady Navier-Stokes equations. SIAM J. Numer. Anal., 32(2):386-403, 1995.

[38] R. Témam. Une méthode d'approximation de la solution des équations de Navier-Stokes. Bull. Soc. Math. Fr., 96:115$152,1968$.

[39] R. Témam. Navier-Stokes Equations and Nonlinear Functional Analysis. CBMS-NSF Regional Conference Series in Applied Mathematics. Society for Industrial and Applied Mathematics, 1983.

[40] R. Témam. Navier-Stokes Equations: Theory \& Numerical Analysis. Studies in Mathematics and Its Applications. NorthHolland, 1984.

[41] H. Yin. Stochastic Navier-Stokes equations with artificial compressibility in random durations. International Journal of Stochastic Analysis, 2010:Article ID 730492, 24 pages, 2010.

(Erika Hausenblas) Lehrstuhl Angewandte Mathematik, Montanuniversität LeOben, Peter-TunNeR-Strasse 25-27, AT-8700 LEOBEN

(Tsiry Randrianasolo) FAKUltät FÜR MATHEMATIK, UNIVERSITÄt BIELEFELD, UNIVERSITÄtSSTRASSE 25, DE-33615

BIELEFELD

E-mail address, Corresponding author: trandria@math.uni-bielefeld.de 